Review

\title{
Power Electronics Converter Technology Integrated Energy Storage Management in Electric Vehicles: Emerging Trends, Analytical Assessment and Future Research Opportunities
}

\author{
Molla Shahadat Hossain Lipu ${ }^{1,2, *(\mathbb{D})}$, Md. Sazal Miah ${ }^{3}(\mathbb{D})$, Shaheer Ansari ${ }^{1}\left(\mathbb{D}\right.$, Sheikh Tanzim Meraj ${ }^{4}(\mathbb{D}$, \\ Kamrul Hasan ${ }^{5}$ (D), Rajvikram Madurai Elavarasan ${ }^{6, *}$, Abdullah Al Mamun ${ }^{7}$, \\ Muhammad Ammirrul A. M. Zainuri ${ }^{1}$ (D) and Aini Hussain ${ }^{1}$ (D)
}

check for updates

Citation: Hossain Lipu, M.S.; Miah, M.S.; Ansari, S.; Meraj, S.T.; Hasan, K.; Elavarasan, R.M.; Mamun, A.A.; Zainuri, M.A.A.M.; Hussain, A. Power Electronics Converter Technology Integrated Energy Storage Management in Electric Vehicles: Emerging Trends, Analytical Assessment and Future Research Opportunities. Electronics 2022, 11, 562. https://doi.org/ 10.3390/electronics 11040562

Academic Editor: Arturo de la Escalera Hueso

Received: 24 December 2021 Accepted: 9 February 2022 Published: 13 February 2022

Publisher's Note: MDPI stays neutral with regard to jurisdictional claims in published maps and institutional affiliations.

Copyright: (C) 2022 by the authors. Licensee MDPI, Basel, Switzerland. This article is an open access article distributed under the terms and conditions of the Creative Commons Attribution (CC BY) license (https:// creativecommons.org/licenses/by/ $4.0 /)$.
1 Department of Electrical, Electronic and Systems Engineering, Universiti Kebangsaan Malaysia, Bangi 43600, Selangor, Malaysia; p100855@siswa.ukm.edu.my (S.A.); ammirrulatiqi@ukm.edu.my (M.A.A.M.Z.); draini@ukm.edu.my (A.H.)

2 Department of Electrical and Electronics Engineering, Green University of Bangladesh, Dhaka 1207, Bangladesh

3 School of Engineering and Technology, Asian Institute of Technology, Khlong Nueng, Pathum Thani 12120, Thailand; st121577@ait.asia

4 Department of Electrical and Electronic Engineering, Universiti Teknologi PETRONAS, Seri Iskandar 32610, Perak, Malaysia; sheikh_19001724@utp.edu.my

5 School of Electrical Engineering, College of Engineering, Universiti Teknologi MARA, Shah Alam 40450, Selangor, Malaysia; 2019984679@isiswa.uitm.edu.my

6 Department of Electrical and Electronics Engineering, Thiagarajar College of Engineering, Madurai 625015, India

7 Department of Electrical and Electronic Engineering, Northern University Bangladesh, Dhaka 1205, Bangladesh; almamun@nub.ac.bd

* Correspondence: lipu@ukm.edu.my (M.S.H.L.); rajvikram787@gmail.com (R.M.E.)

\begin{abstract}
Globally, the research on electric vehicles (EVs) has become increasingly popular due to their capacity to reduce carbon emissions and global warming impacts. The effectiveness of EVs depends on appropriate functionality and management of battery energy storage. Nevertheless, the battery energy storage in EVs provides an unregulated, unstable power supply and has significant voltage drops. To address these concerns, power electronics converter technology in EVs is necessary to achieve a stable and reliable power transmission. Although various EV converters provide significant contributions, they have limitations with regard to high components, high switching loss, high current stress, computational complexity, and slow dynamic response. Thus, this paper presents the emerging trends in analytical assessment of power electronics converter technology incorporated energy storage management in EVs. Hundreds (100) of the most significant and highly prominent articles on power converters for EVs are studied and investigated, employing the Scopus database under predetermined factors to explore the emerging trends. The results reveal that $57 \%$ of articles emphasize modeling, experimental work, and performance evaluation. In comparison, $13 \%$ of papers are based on problem formulation and simulation analysis, and $8 \%$ of articles are survey, case studies, and review-based. Besides, four countries, including China, India, the United States, and Canada, are dominant to publish the maximum articles, indicating 33,17,14, and 13, respectively. This review adopts the analytical assessment that outlines various power converters, energy storage, controller, optimization, energy efficiency, energy management, and energy transfer, emphasizing various schemes, key contributions, and research gaps. Besides, this paper discusses the drawbacks and issues of the various power converters and highlights future research opportunities to address the existing limitations. This analytical assessment could be useful to EV engineers and automobile companies towards the development of advanced energy storage management interfacing power electronics for sustainable EV applications.
\end{abstract}

Keywords: power electronics; energy storage management; electric vehicles; emerging trends analytical assessment 


\section{Introduction}

In recent years, humankind has faced one of the biggest challenges in terms of climate change, which is to deal with the emission of carbon dioxide due to a need to supply the increasing energy demand to support the industrial growth around the globe [1]. Globally, poor air quality in developing countries such as China is a significant and growing problem. As the economies of developing nations grow, the increased demand for automobiles and freight movement will only make these problems more difficult to address. One of the primary causes of global warming and climate change is the rising usage of fossil fuels in diesel-powered automobiles [2,3]. The increasing demand for energy consumption and dependency on carbon-based energy in the automotive industries in the last decade has become one of the most significant concerns around the globe. It was observed from different surveys that more than 500 million metric tons of carbon-based elements are emitted from the transportation sector only [4]. To tackle this major issue, electric vehicles (EVs) have appeared as a blessing to support building a totally sovereign society of carbonbased energy consumption and usage [5]. Unlike conventional vehicles, which run on gasoline or diesel, fuel cell cars and trucks combine hydrogen and oxygen to produce electricity. As a result, they have low energy densities and high energy efficiencies. Internalcombustion engines, which have hundreds of moving parts, are getting rapidly replaced to EVs with typically less than 20 moving parts, eliminating the need for conventional automobiles. Energy storages (ESs), traction motors, and power electronics are the new focal points of innovation in EVs [6]. The demand for greater vehicle range, safety, lifetime, and, of course, sustainable transportation drives technological developments in these components $[7,8]$.

In EVs, power electronics largely process and control the flow of electrical energy [9]. They also regulate the motor's speed and the torque it generates. Finally, power electronics transform and distribute electrical power to other vehicle systems like heating, ventilation, lighting, and information technology [10]. Inverters, DC-DC converters, and chargers are examples of power electronics components [11,12]. Perfect synchronization between ESs and power electronic converters is key to the efficient performance of EVs [13]. The application and interfacing of ESs and power electronic converters in EVs can be further understood by the basic illustration of an EV shown in Figure 1.

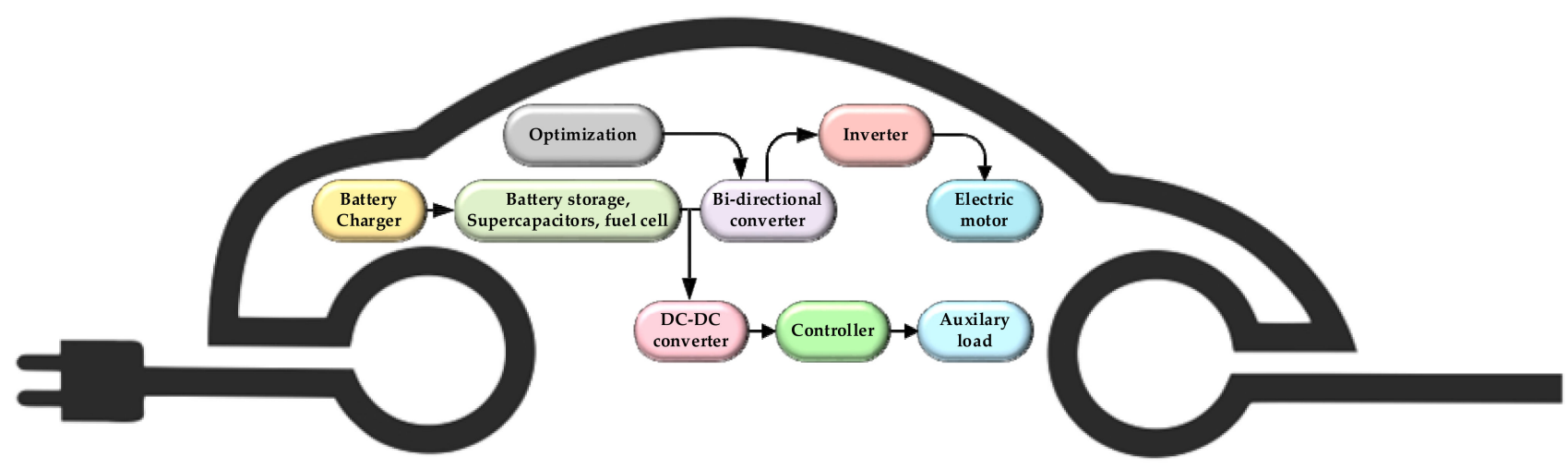

Figure 1. Schematic illustration of the application and interfacing of power electronic converters and ESs in EVs.

Various types of ESs, including supercapacitors, batteries, fuel cells, are integrated with power electronic converters/inverters to develop the internal structure of EVs [14-17]. In EVs, an AC-DC inverter is required to convert DC energy from ESs to AC power to drive the motor. An inverter also serves as a motor controller and a filter, protecting the ESs from stray current damage. The electricity delivered by ESs, on the other hand, has unreliable characteristics and significant voltage drops. DC-DC converters effectively tackle this issue. To accommodate the voltage needs of motors of EVs, DC-DC converters are 
used to improve ESs voltage profile. A boost converter is required if the EV's motor design requires higher voltage, such as an internal permanent magnet motor. As a result, DC-DC converters are critical for regulated power flow in EVs [18-21]. However, the enormous size of the capacitor storage on the DC link is one of the major issues with boost converters. In addition, these converters have the drawback of injecting discontinuous current into the load. Furthermore, the capacitor's ripple current is roughly equivalent to the load current. As a result, the capacitor tank's Externally Supported Roof (ESR) regulation is fairly stringent. This is especially true in new EV power conversion application domains, where the input voltage is generally very low and, equally, a huge voltage boost is required.

The primary goal of utilizing a controller in an EV converter is to achieve performance enhancement characteristics, including better and faster steady-state and dynamic response, reduced settling time, and reduced steady-state faults [22,23]. The better dynamic response eventually leads to reduced electromagnetic interference (EMI), switching losses, and common-mode voltage (CMV). The DC-DC converters in EVs are controlled by various linear and non-linear controllers [24]. Linear controllers have a basic design and are simple to implement. Nevertheless, their performance significantly deteriorates when the design parameters are changed or in the presence of load disturbances $[25,26]$. Intelligent controllers are being used to overcome these issues, and they have been successful in providing an exceptionally dynamic response with better stability and higher efficiency [27]. Intelligent controllers can also handle systems that are highly non-linear and complicated [28-30].

In EV converters, the primary objective of the optimization technique is to optimize the converter's structure and enhance other characteristics while considering a variety of limitations [31-33]. The converter's design can be improved by reducing the number of power electronic components and making the converter economical. Accordingly, the converter's efficiency can be enhanced by lowering switching and conduction loss, switching angle, and voltage/current ripple [34]. A multi-objective function aids in the achievement of all requirements for developing an optimized converter for EVs [35]. The design of a multi-objective function, on the other hand, is a time-consuming task that necessitates the assignment of numerous parameters, variables, and constraints, such as switching frequency, duty cycle, components count, switching angles, and input voltage/current. Akkaldevi et al. [36] introduces the quantitative validation carried out on a prismatic $20 \mathrm{Ah} \mathrm{LiFePO} 4$ battery sandwiched between two mini channel cold plates. Purohit et al. [37] two-layer feed-forward artificial neural-network-based machine learning to design soft sensors. Subsequently, estimate the state of charge (SOC), state of energy (SOE), and power loss (PL) of a formula student electric vehicle (FSEV) battery-pack system. Choudhari et al. [38] discuss the thermal performance of the battery module, which contains $5 \times 5$ li-ion battery arranged in series and parallel. In terms of battery equalization and self-heating, various authors widely utilized different strategies such as Integrated Self-Heater [39], Automotive Battery Equalizers [40], compact resonant switched-capacitor heater [41], and sine-wave heating circuit [42]. Apart from this, various reviewers believe that usage of some interpretable tool will be beneficial for power electronics integrated energy storage management [43,44].

The topological reviews on DC-DC converters and their controls have been carried out in numerous manuscripts, especially on EVs and renewable energy applications. The objectives, focused areas, and research gaps of these articles are summarized in Table 1. Chakraborty et al. [45] investigated different topologies of DC-DC converters used in EVs, giving additional attention to converter topologies and their evaluation. However, they omitted to review the DC-DC converters' controller and optimization approaches and related implementation challenges in EVs. This shortcoming was overcome by the review article of Anbazhagan et al. [31], which provided a detailed review on converters for EVs, emphasizing both isolated and non-isolated converters; nevertheless, control, function, optimization, and modulation were not covered in depth. The review article [46] provided an extensive overview of the multi-input DC-DC converters for EVs. However, it did 
not include the issues associated with implementing these converters in EV applications. Different DC-DC converters in EV applications were described and compared [47]. However, the converter's description was confined to limited control methods. Furthermore, like [31], the manuscript failed to provide insight into the DC-DC converters' operation, optimization, and modulation. Therefore, it can be stated that the majority of these articles failed to provide an effective review of the control and performance optimization of DC-DC converters for EV applications. Thus, this review provides a detailed analysis and evaluation of various power electronics converters in the relevant 100 papers highlighting research trends, critical analyses, contributions, and research gaps. This review adopts a statistical approach to analyze publication year, authorship, journals, publisher, university, country, and collaboration. In addition, this review covers various key aspects, including comparative analysis among power electronic converters, types of the energy storage system, benefits of artificial intelligence (AI), controllers, and optimization in EV power converters. Furthermore, numerous key issues and challenges are explored, and accordingly, effective suggestions and insightful remarks are outlined.

Table 1. The notable studies on EV power electronics converters, focused areas, and research gaps.

\begin{tabular}{|c|c|c|c|}
\hline Objectives & Focused Areas & Research Gaps & Ref \\
\hline $\begin{array}{l}\text { Reviewing DC-DC } \\
\text { converters for EVs }\end{array}$ & $\begin{array}{l}\text { Focus on the converter topologies for } \\
\text { plugged-in hybrid vehicles. } \\
\text { A thorough review of converters for } \\
\text { charging stations. }\end{array}$ & $\begin{array}{l}\text { - No information on the } \\
\text { converter's controllers. } \\
\text { Omitted to review the converter's } \\
\text { optimization methods. }\end{array}$ & {$[45]$} \\
\hline $\begin{array}{l}\text { Reviewing isolated and } \\
\text { non-isolated converters } \\
\text { for EVs }\end{array}$ & $\begin{array}{l}\text { Detailed review on the isolated and } \\
\text { non-isolated converters for } \\
\text { EV applications. }\end{array}$ & $\begin{array}{l}\text { - No emphasis was given on the } \\
\text { converters' control, operation, } \\
\text { performance, or optimization. }\end{array}$ & {$[48,49]$} \\
\hline $\begin{array}{l}\text { Reviewing DC-DC } \\
\text { converters for EVs }\end{array}$ & $\begin{array}{l}\text { A topological comparison of the } \\
\text { converters is presented in terms of } \\
\text { EV applications. } \\
\text { - Some control methods are analyzed. }\end{array}$ & $\begin{array}{l}\text { Only a few control methods of } \\
\text { converters were discussed. } \\
\text { No emphasis was given on the } \\
\text { performance and optimization } \\
\text { of converters. } \\
\text { Issues and challenges of implementing } \\
\text { converters are not discussed. }\end{array}$ & [47] \\
\hline $\begin{array}{l}\text { Reviewing } \\
\text { multi-input DC-DC } \\
\text { converters for EVs. } \\
\text { Reviewing energy } \\
\text { sources for EVs. }\end{array}$ & $\begin{array}{l}\text { Detailed review on different multi-input } \\
\text { converter topologies for EV and } \\
\text { renewable energy applications. } \\
\text { Cost analysis on the energy sources for } \\
\text { EV applications. }\end{array}$ & $\begin{array}{l}\text { - Only multi-input converters } \\
\text { are considered. } \\
\text { No emphasis was given on the } \\
\text { converters' control, operation, } \\
\text { performance, or optimization. } \\
\text { Issues and challenges of implementing } \\
\text { converters are not discussed. }\end{array}$ & [50-52] \\
\hline
\end{tabular}

- A comprehensive review of various converters for EVs.

Reviewing multi-input DC-DC converters and their controls for battery charge balancing in EVs.
- A comprehensive review of the control methods of converters for EV applications.

- Issues and future trends associated with converters'based battery charge balancing in EVs are discussed briefly.
- No discussion is provided on the controller optimization and performance of the converters. 
Table 1. Cont.

\begin{tabular}{|c|c|c|c|}
\hline Objectives & Focused Areas & Research Gaps & Ref \\
\hline $\begin{array}{l}\text { Reviewing non } \\
\text { isolated converters } \\
\text { for EVs. } \\
\text { Reviewing energy } \\
\text { sources for EVs. }\end{array}$ & $\begin{array}{l}\text { Detailed review and comparative } \\
\text { analysis on the non-isolated converters } \\
\text { for EV applications. } \\
\text { Implementation of various energy } \\
\text { sources in practical EVs are } \\
\text { discussed extensively. }\end{array}$ & $\begin{array}{l}\text { - No emphasis was given on the } \\
\text { converters' control, operation, } \\
\text { performance, or optimization. } \\
\text { Issues and challenges of implementing } \\
\text { converters are not discussed. }\end{array}$ & [55] \\
\hline $\begin{array}{l}\text { Reviewing DC-DC } \\
\text { converters for battery } \\
\text { storage systems in EVs } \\
\text { and grid applications. }\end{array}$ & $\begin{array}{l}\text { A comprehensive review on converters } \\
\text { for battery storage systems in EVs and } \\
\text { grid systems. } \\
\text { Focus on the charge control methods of } \\
\text { batteries for EVs and grids. } \\
\text { Issues and future recommendations for } \\
\text { implementing converters in EVs are } \\
\text { discussed briefly. }\end{array}$ & $\begin{array}{l}\text { - No emphasis was given on the } \\
\text { converters' control, operation, } \\
\text { performance, or optimization. }\end{array}$ & [56] \\
\hline
\end{tabular}

Analytical assessment is a type of research article that combines the library and information science with statistics and quantitative approaches to provide information and analysis in a variety of formats $[57,58]$. Emerging trends and analytical evaluation are important areas because they provide historical and extensive information that can be used to address future challenges and provide appropriate recommendations $[59,60]$. Analytical assessment can be used by researchers, higher education institutions, and their respective students and lecturers to evaluate a journal article's quality utilizing a range of key factors like citations, h-index, impact factors, and quartiles. Gingras [61] looked at the impact of analytical assessment on research objectives and offered a few guidelines and criteria for building an appropriate assessment technique for a certain research plan and analysis size. Andres [62] discussed the procedures, significance and impacts of analytical analysis in real-world applications. This paper performs an analytical analysis to look at the present state of published activity in power electronic DC-DC converters for EV applications.

In literature, some analytical assessment articles can be found that are based on EVs such as reliability of EVs [63], fuzzy optimization control based EVs [64], autonomous vehicles [65], optimization of energy management schemes in EVs [66], advanced and next-generation EVs [67], EV battery industries' expansion in China [68] are just a few of the analytical assessment analyses conducted on EVs and their applications. As of now, no analytical analysis of DC-DC power converters for EV applications has been performed, to our knowledge. Therefore, this study describes the first analytical evaluation of power converters applied in EV applications, which was conducted from the year 2010 until November 2021 to look at the articles, investigation, achievements, and innovation, and recent advances in this field. The key findings and overall contributions of this paper are highlighted below.

- In terms of the number of articles published to date, a brief synopsis of DC-DC converters for EV applications is presented. The analysis is carried out once a year and is followed by a discussion.

- Power converters for EV applications are analyzed using the most cited authors, the universities with the highest number of articles, and the nation with the most published articles on the discussed topic. This is vital for determining author, organization, and country productivity in the discussed topic and enhancing research production and collaboration among authors.

- The keywords and topics used in the content analysis and gap analysis are assessed.

- Original manuscripts, review articles, and book chapters are among the studied document categories. The impact factors of the journals and the reputation of the publishers in the research community are also analyzed. 
- The extent of collaboration between researchers is identified. The number of authors in the manuscripts and the links between different universities and countries are utilized to evaluate the team.

The emerging trends and analytical assessment aim to explore the 100 most significant publications in power electronics converter (PEC) for EV applications. Accordingly, complete and detailed information, critical debates, facts, analyses, achievements and shortcomings, issues, and challenges of these publications are presented. This review offers several benefits, including,

- To provide the history, current trends, evolution, applications, and future research opportunities of PEC in EV applications.

- $\quad$ To deliver a comparative study of the most appropriate papers for PEC in EVs that will support the future expansion of present knowledge, experience, and implementation.

- Finally, this analytical assessment provides constructive suggestions for the development and prospects of PEC in EV applications.

This review is structured into six sections. Section 1 describes an outline of the PEC and analytical assessment along with contributions and research gaps; Section 2 highlights the approaches and procedures of the surveying methodology for analytical evaluation; Section 3 covers an in-depth investigation of the selected manuscripts on PEC in EV applications. Section 4 explores the numerous existing issues, limitations, and issues. The future research opportunities and suggestions are depicted in Section 5, while Section 6 delivers the conclusion.

\section{Survey Methods}

This work was conducted in end of November, 2021 using the bibliometric technique, a statistical analysis of the Scopus database (www.scopus.com, accessed on 5 November 2021). The Scopus database was utilized as an article source in this bibliometric study because it has a greater quantity of publications than other platforms and databases, such as Web of Science [69]. Google Scholar is not examined in this study [70] due to a lack of credible results. The analysis was taken place at the end of November 2021. The bibliometric analysis approaches used in the Scopus database are illustrated in Figure 2. The technique is separated into five steps, as shown in the diagram below:

\subsection{Inclusion and Exclusion Criteria}

The papers were chosen using predetermined conditions from the Scopus database. The fundamental searching keywords code for article search from the Scopus database is shown in Table 2. For the 100 most noteworthy publications in the area of PEC technology integrated energy storage management (ESM) in EV applications, the following criteria for article inclusion and exclusion were used:

- Keywords like converter, energy storage, and electric vehicle were used as the major criterion for paper inclusion. Some articles were excluded manually due to the field's insignificance.

- Manuscripts written in the English language that were published between 2010 and 2021 were analyzed for this bibliometric analysis.

\subsection{Screening Procedures}

As the number of published papers varies under different databases, the following criteria and procedures were utilized to find the most appropriate publication from the Scopus database.

- $\quad$ A total of $1954(n=1954)$ manuscripts were selected from the fundamental selection process.

- A total of $1704(n=1704)$ research papers were selected after employing year limitation range from 2010 to 2021.

- By defining topic areas, a total of $1688(n=1688)$ articles were chosen. 
- Then, a total of 659 articles were selected by limiting document type (Article and Review).

- By applying the "English Language" filter, a total of $612(n=612)$ articles were chosen.

- Finally, on the basis of relevancy, a total of $100(n=100)$ manuscripts were selected for the final evaluation from the Scopus database.

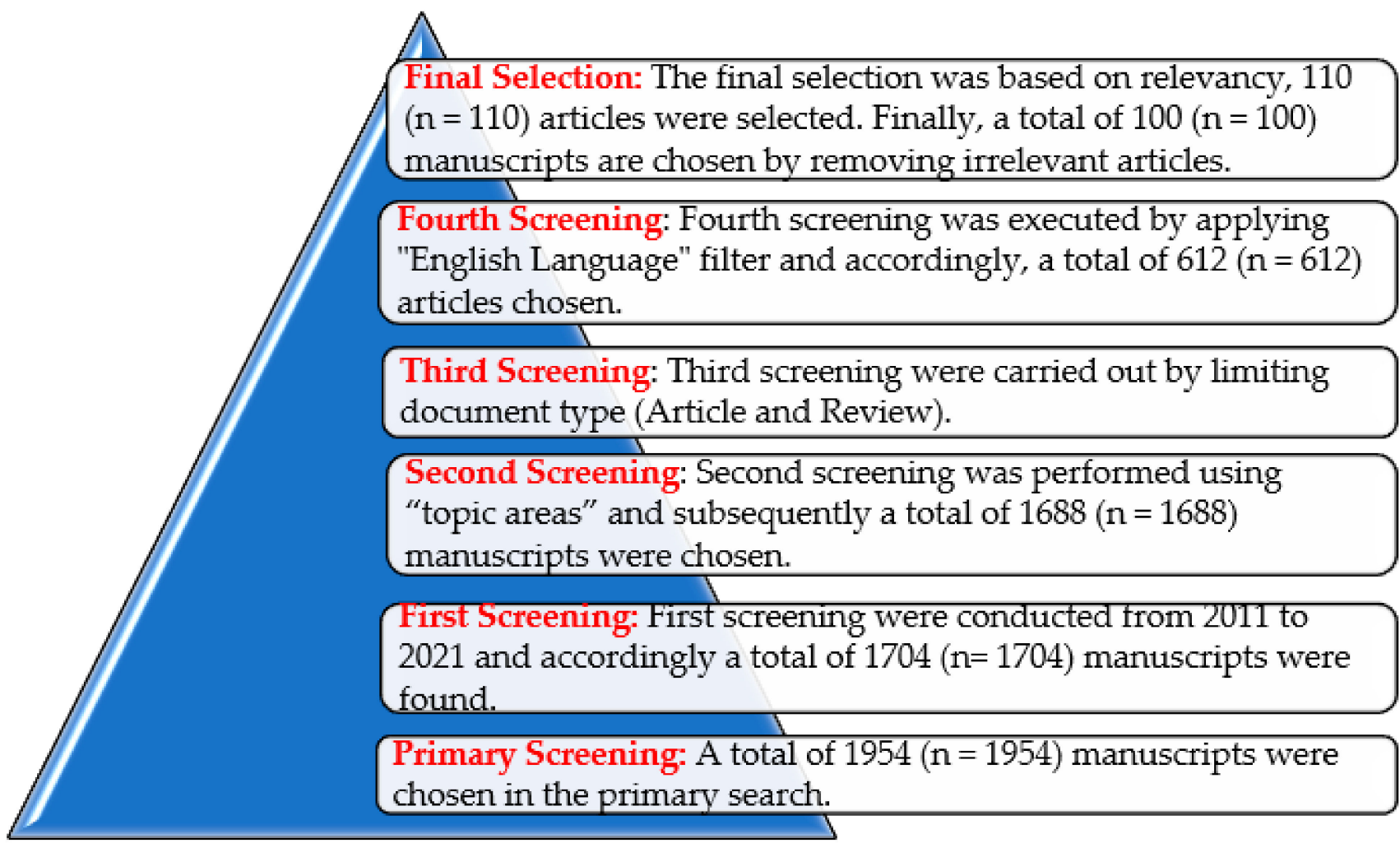

Figure 2. Manuscript's selection stages from Scopus database.

Table 2. Searching codes to find the most relevant 100 manuscripts from the Scopus database.

\begin{tabular}{|c|c|c|c|}
\hline Step & $\begin{array}{l}\text { Types of } \\
\text { Filtering }\end{array}$ & Search Code & $\begin{array}{l}\text { Number of } \\
\text { Manuscripts }\end{array}$ \\
\hline Primary & $\begin{array}{l}\text { By keyword: } \\
\text { Converter } \\
\text { Energy Storage } \\
\text { Electric Vehicle }\end{array}$ & TITLE-ABS-KEY (converter AND energy AND storage AND electric AND vehicle) & 1954 \\
\hline 2nd & Year (2010-2022) & $\begin{array}{l}\text { TITLE-ABS-KEY (converter AND energy AND storage AND electric AND vehicle) } \\
\text { AND (LIMIT-TO (PUBYEAR, 2022) OR LIMIT-TO (PUBYEAR, 2021) OR } \\
\text { LIMIT-TO (PUBYEAR, 2020) OR LIMIT-TO (PUBYEAR, 2019) OR LIMIT-TO } \\
\text { (PUBYEAR, 2018) OR LIMIT-TO (PUBYEAR, 2017) OR LIMIT-TO (PUBYEAR, } \\
\text { 2016) OR LIMIT-TO (PUBYEAR, 2015) OR LIMIT-TO (PUBYEAR, 2014) OR } \\
\text { LIMIT-TO (PUBYEAR, 2013) OR LIMIT-TO (PUBYEAR, 2012) OR LIMIT-TO } \\
\text { (PUBYEAR, 2011) OR LIMIT-TO (PUBYEAR, 2010)) }\end{array}$ & 1704 \\
\hline
\end{tabular}


Table 2. Cont.

\begin{tabular}{|c|c|c|c|}
\hline Step & $\begin{array}{l}\text { Types of } \\
\text { Filtering }\end{array}$ & Search Code & $\begin{array}{l}\text { Number of } \\
\text { Manuscripts }\end{array}$ \\
\hline $3 \mathrm{rd}$ & Subject area & $\begin{array}{l}\text { TITLE-ABS-KEY (converter AND energy AND storage AND electric AND vehicle) } \\
\text { AND (LIMIT-TO (PUBYEAR, 2022) OR LIMIT-TO (PUBYEAR, 2021) OR LIMIT-TO } \\
\text { (PUBYEAR, 2020) OR LIMIT-TO (PUBYEAR, 2019) OR LIMIT-TO (PUBYEAR, } \\
\text { 2018) OR LIMIT-TO (PUBYEAR, 2017) OR LIMIT-TO (PUBYEAR, 2016) OR } \\
\text { LIMIT-TO (PUBYEAR, 2015) OR LIMIT-TO (PUBYEAR, 2014) OR LIMIT-TO } \\
\text { (PUBYEAR, 2013) OR LIMIT-TO (PUBYEAR, 2012) OR LIMIT-TO (PUBYEAR, } \\
\text { 2011) OR LIMIT-TO (PUBYEAR, 2010)) AND (LIMIT-TO (SUBJAREA, “ENGI”) } \\
\text { OR LIMIT-TO (SUBJAREA, “ENER") OR LIMIT-TO (SUBJAREA, “COMP”) OR } \\
\text { LIMIT-TO (SUBJAREA, “MATH”) OR LIMIT-TO (SUBJAREA, “PHYS”) OR } \\
\text { LIMIT-TO (SUBJAREA, “ENVI") OR LIMIT-TO (SUBJAREA, “MATE”) OR } \\
\text { LIMIT-TO (SUBJAREA, “CENG”) OR LIMIT-TO (SUBJAREA, “CHEM”)) }\end{array}$ & 1688 \\
\hline 4 th & $\begin{array}{l}\text { Document Type } \\
\text { (Article } \\
\text { and Review) }\end{array}$ & $\begin{array}{l}\text { TITLE-ABS-KEY (converter AND energy AND storage AND electric AND vehicle) } \\
\text { AND (LIMIT-TO (PUBYEAR, 2022) OR LIMIT-TO (PUBYEAR, 2021) OR } \\
\text { LIMIT-TO (PUBYEAR, 2020) OR LIMIT-TO (PUBYEAR, 2019) OR LIMIT-TO } \\
\text { (PUBYEAR, 2018) OR LIMIT-TO (PUBYEAR, 2017) OR LIMIT-TO (PUBYEAR, } \\
\text { 2016) OR LIMIT-TO (PUBYEAR, 2015) OR LIMIT-TO (PUBYEAR, 2014) OR } \\
\text { LIMIT-TO (PUBYEAR, 2013) OR LIMIT-TO (PUBYEAR, 2012) OR LIMIT-TO } \\
\text { (PUBYEAR, 2011) OR LIMIT-TO (PUBYEAR, 2010)) AND (LIMIT-TO (SUBJAREA, } \\
\text { “ENGI") OR LIMIT-TO (SUBJAREA, “ENER") OR LIMIT-TO (SUBJAREA, } \\
\text { “COMP”) OR LIMIT-TO (SUBJAREA, “MATH”) OR LIMIT-TO (SUBJAREA, } \\
\text { “PHYS") OR LIMIT-TO (SUBJAREA, “ENVI") OR LIMIT-TO (SUBJAREA, } \\
\text { “MATE") OR LIMIT-TO (SUBJAREA, “CENG”) OR LIMIT-TO (SUBJAREA, } \\
\text { “CHEM")) AND (LIMIT-TO (DOCTYPE, “ar") OR LIMIT-TO (DOCTYPE, "re")) }\end{array}$ & 659 \\
\hline 5th & $\begin{array}{l}\text { Language } \\
\text { (English) }\end{array}$ & $\begin{array}{l}\text { TITLE-ABS-KEY (converter AND energy AND storage AND electric AND vehicle) } \\
\text { AND (LIMIT-TO (PUBYEAR, 2022) OR LIMIT-TO (PUBYEAR, 2021) OR } \\
\text { LIMIT-TO (PUBYEAR, 2020) OR LIMIT-TO (PUBYEAR, 2019) OR LIMIT-TO } \\
\text { (PUBYEAR, 2018) OR LIMIT-TO (PUBYEAR, 2017) OR LIMIT-TO (PUBYEAR, } \\
\text { 2016) OR LIMIT-TO (PUBYEAR, 2015) OR LIMIT-TO (PUBYEAR, 2014) OR } \\
\text { LIMIT-TO (PUBYEAR, 2013) OR LIMIT-TO (PUBYEAR, 2012) OR LIMIT-TO } \\
\text { (PUBYEAR, 2011) OR LIMIT-TO (PUBYEAR, 2010)) AND (LIMIT-TO (SUBJAREA, } \\
\text { “ENGI") OR LIMIT-TO (SUBJAREA, “ENER") OR LIMIT-TO (SUBJAREA, } \\
\text { “COMP”) OR LIMIT-TO (SUBJAREA, “MATH”) OR LIMIT-TO (SUBJAREA, } \\
\text { "PHYS”) OR LIMIT-TO (SUBJAREA, “ENVI") OR LIMIT-TO (SUBJAREA, } \\
\text { “MATE") OR LIMIT-TO (SUBJAREA, “CENG”) OR LIMIT-TO (SUBJAREA, } \\
\text { “CHEM")) AND (LIMIT-TO (DOCTYPE, “ar") OR LIMIT-TO (DOCTYPE, "re”)) } \\
\text { AND (LIMIT-TO (LANGUAGE, “English")) }\end{array}$ & 612 \\
\hline
\end{tabular}

\subsection{Research Trend}

To develop more efficient ESM for EV applications, researchers are currently doing lots of research on PEC [71-80]. The researchers use different power electronics converters to provide more effective energy storage management. Figure 3 shows the research trend from 2010 to 2021, showing an upward trend. The number of published articles rises along with time, which means researchers are more focused on the field of PEC, as seen in Figure 3. A total of 1954 articles were published between 2010 and 2021. The figure shows a constant growth in terms of the number of publications. Apart from this, the figure illustrates a different trend after 2012, and the figure was 78 in 2013, which was lower than the previous year's total publication. According to Figure 3, the research articles in the area of PEC integrated ESM in EV applications have increased linearly along with time.

\subsection{Data Extraction}

The following criteria were used to extract information from the selected most relevant 100 manuscripts: name of the authors, types of converters, abbreviated keywords, types of manuscripts (original research work and review paper), name of the journal, publisher name, publication year, name of the corresponding authors affiliated country, 
and the number of total citations. Following the study of the data from the selected manuscript, a conclusion is drawn to offer a complete picture of energy storage strategies for EV applications.

\subsection{Study Characteristics and Findings}

The Scopus database yielded a total of 1954 papers from the elementary search. The highly appropriate 100 manuscripts were selected and depicted in Table 2 with the name of the authors, types of converters, abbreviated keywords, types of manuscripts (original research work and review paper), name of the journal, publisher name, publication year, name of the corresponding authors' affiliated country, and the number of total citations, respectively. The selected manuscripts have a total of 4014 citations (mean 40.14; median 18.5; and citation range 0 to 809). In addition, 11 of the 100 articles received over 100 citations. With 809 citations and an impact factor (IF) of 6.153, Cao et al. [81] authored the most cited manuscripts in the "IEEE Transactions on Power Electronics" journal in 2012.

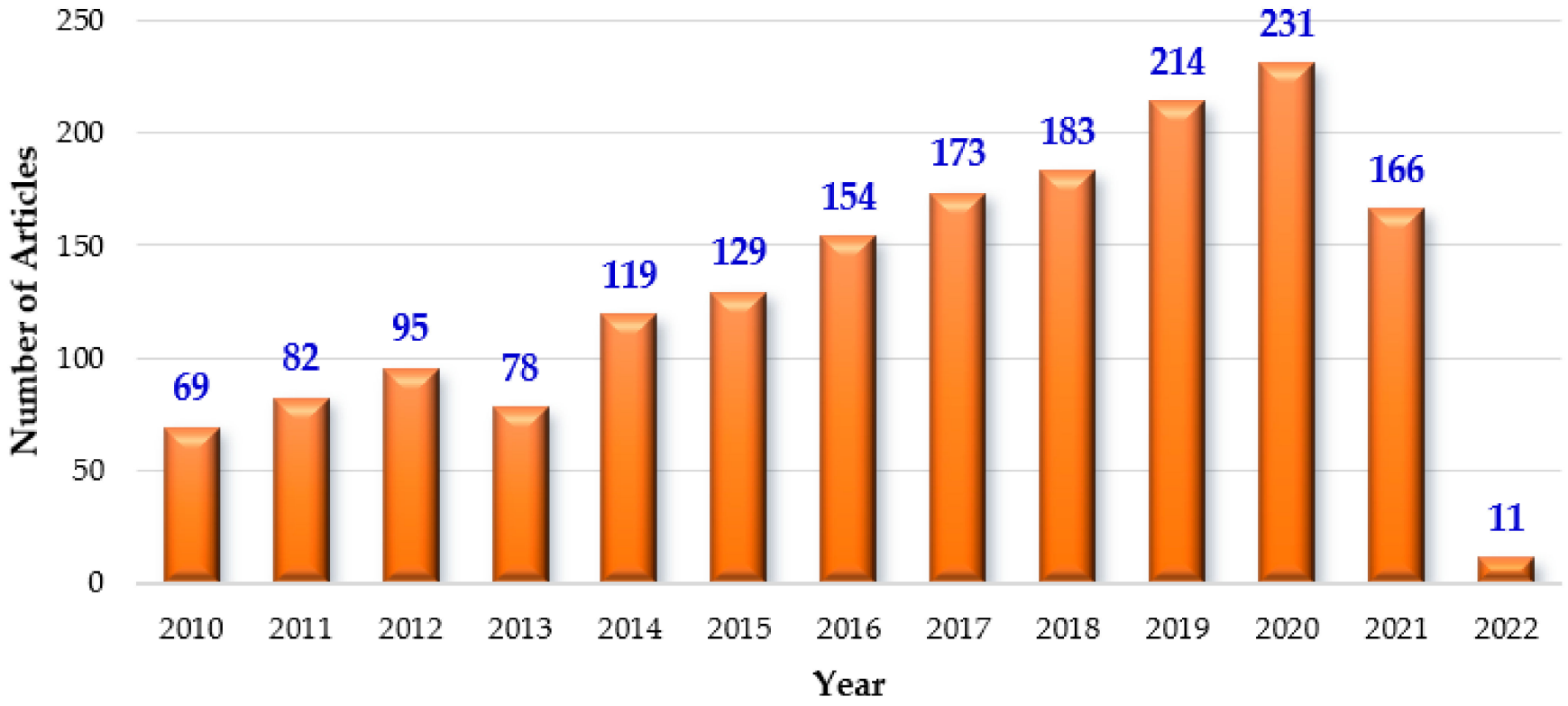

Figure 3. Conducted research works on the field of PEC integrated ESM for EV applications between 2010 and 2022.

\section{Analytical Discussion}

The most relevant article analysis in a certain field is important for categorizing and understanding current research trends and providing a solid indication of the influence of a journal. This analytical discussion aims to provide a clear indication about the most valuable articles, recent research trends, outcomes, critical discussion in PEC integrated ESM in EVs.

\subsection{Citation Evaluation of the Highly Influential Publications}

The 100 most influential publications in the area of PEC integrated ESM in EV application, as collected from the Scopus database, are depicted in Table 3. It is noticed from Table 3 that the number of citations for the 100 selected relevant manuscripts ranged from 0 to 809; the first three manuscripts each had above 150 citations, and the initial 11 papers each contained 100 citations. Cao et al. published the paper with the greatest number of citations in 2012, while Fang et al. produced the manuscript with the lowest number of citations in 2021. 
Table 3. The most relevant 100 articles in the field of PEC integrated ESM in EV applications.

\begin{tabular}{|c|c|c|c|c|c|c|c|c|c|c|c|}
\hline Rank & Ref. & Authors & Converter Type & Energy Storage Type & Author Keywords & Article Type & $\begin{array}{c}\text { Journal } \\
\text { Abbreviation }\end{array}$ & Publisher & Year & Country & Citation \\
\hline 1 & [81] & Cao et al. & $\begin{array}{c}\text { a larger } \\
\text { DC-DC converter }\end{array}$ & $\begin{array}{l}\text { Hybrid Energy } \\
\text { Storage System }\end{array}$ & $\begin{array}{c}\text { Battery; Control; DC/DC converters; } \\
\text { EV; ES; HEV; PHEV; PE; propulsion } \\
\text { systems; UC }\end{array}$ & Article & ITPEE & IEEE & 2012 & $\begin{array}{l}\text { United } \\
\text { States }\end{array}$ & 809 \\
\hline 2 & [82] & Song et al. & $\begin{array}{l}\text { Unidirectional } \\
\text { DC-DC converter }\end{array}$ & $\begin{array}{l}\text { Hybrid Energy } \\
\text { Storage System }\end{array}$ & $\begin{array}{l}\text { EV; HESS; LiFePO4 battery degradation; } \\
\text { multi-objective optimization }\end{array}$ & Article & APEND & Elsevier Ltd. & 2014 & China & 193 \\
\hline 3 & [83] & Zheng et al. & $\begin{array}{l}\text { a hybrid cascaded } \\
\text { multilevel converter }\end{array}$ & Battery Storage & $\begin{array}{l}\text { BC; charging and discharging; EV; } \\
\text { hybrid cascaded multilevel converter; } \\
\text { voltage balance }\end{array}$ & Article & ITPEE & IEEE & 2014 & China & 168 \\
\hline 4 & [84] & $\begin{array}{l}\text { Nahavandi } \\
\text { et al. }\end{array}$ & $\begin{array}{l}\text { a new non isolated } \\
\text { multi-input } \\
\text { multioutput DC-DC } \\
\text { boost converter }\end{array}$ & $\begin{array}{l}\text { Hybrid Energy } \\
\text { Storage System }\end{array}$ & $\begin{array}{l}\text { DC-DC converters; EV; hybrid power } \\
\text { system; MIMO; small-signal modeling; } \\
\text { state-space averaging }\end{array}$ & Article & ITPEE & IEEE & 2015 & Iran & 148 \\
\hline 5 & [85] & Laldin et al. & $\begin{array}{l}\text { DC-DCDC-DC } \\
\text { converter }\end{array}$ & $\begin{array}{l}\text { Hybrid Energy } \\
\text { Storage System }\end{array}$ & $\begin{array}{l}\text { BMS; DC-DC converters; DC; EVs; EM; } \\
\text { HESS; UC }\end{array}$ & Article & ITPEE & IEEE & 2013 & $\begin{array}{l}\text { United } \\
\text { States }\end{array}$ & 144 \\
\hline 6 & [86] & Samosir et al. & $\begin{array}{l}\text { a bidirectional } \\
\text { DC-DCDC-DC } \\
\text { converter }\end{array}$ & $\begin{array}{l}\text { Hybrid Energy } \\
\text { Storage System }\end{array}$ & $\begin{array}{l}\text { BDC; dynamic evolution control; FC; } \\
\text { UC; ES }\end{array}$ & Article & ITIED & IEEE & 2010 & Indonesia & 133 \\
\hline 8 & [88] & Blanes et al. & $\begin{array}{c}\text { interleaved } \\
\text { bidirectional } \\
\text { buck-boost converter }\end{array}$ & $\begin{array}{l}\text { Hybrid Energy } \\
\text { Storage System }\end{array}$ & $\begin{array}{l}\text { Battery; EV; EM; field-programmable } \\
\text { gate array; interleaved DC-DC } \\
\text { converter; UC }\end{array}$ & Article & ITPEE & IEEE & 2013 & Spain & 128 \\
\hline 9 & [89] & Song et al. & $\begin{array}{l}\text { two bi-directional } \\
\text { DC-DC converters }\end{array}$ & $\begin{array}{l}\text { Hybrid Energy } \\
\text { Storage System }\end{array}$ & $\begin{array}{l}\text { EV; HESS; Lyapunov function-based } \\
\text { controller; sliding-mode controller; SC }\end{array}$ & Article & ENEYD & Elsevier Ltd. & 2017 & China & 109 \\
\hline 10 & [90] & Akar et al. & $\begin{array}{l}\text { a novel bidirectional } \\
\text { non isolated } \\
\text { multi-input converter }\end{array}$ & $\begin{array}{l}\text { Hybrid Energy } \\
\text { Storage System }\end{array}$ & $\begin{array}{l}\text { Batteries; bidirectional; HESSs; } \\
\text { multi-input converter; UC }\end{array}$ & Article & ITVTA & IEEE & 2016 & Turkey & 109 \\
\hline 11 & [91] & Rubino et al. & - & Battery Storage & $\begin{array}{l}\text { ESS; PEV charging infrastructure; PEV; } \\
\text { PEC; SG }\end{array}$ & Article & APEND & Elsevier Ltd. & 2017 & Italy & 104 \\
\hline 12 & [92] & Wang et al. & DC-DC converter & $\begin{array}{l}\text { Hybrid Energy } \\
\text { Storage System }\end{array}$ & $\begin{array}{l}\text { Direct current to direct current } \\
\text { converter; EV; EMS; HESS }\end{array}$ & Article & JPSOD & Elsevier B.V. & 2015 & China & 90 \\
\hline 13 & [93] & Ahrabi et al. & $\begin{array}{l}\text { a multi-input } \\
\text { dc-dc converter }\end{array}$ & Fuel Cell Storage & HEV; multi-input converter; PM & Article & ITPEE & IEEE & 2017 & Iran & 81 \\
\hline
\end{tabular}


Table 3. Cont.

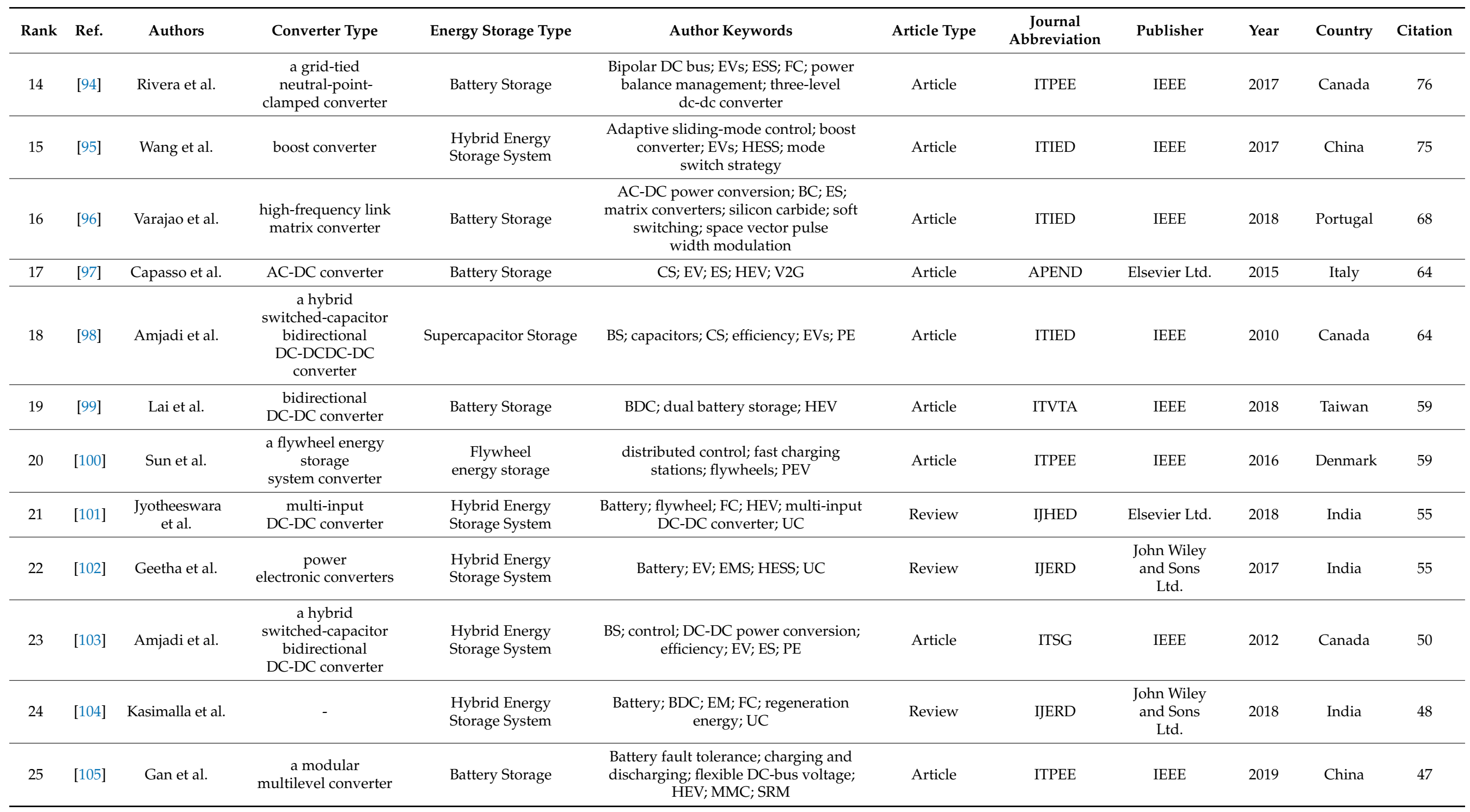


Table 3. Cont.

\begin{tabular}{|c|c|c|c|c|c|c|c|c|c|c|c|}
\hline Rank & Ref. & Authors & Converter Type & Energy Storage Type & Author Keywords & Article Type & $\begin{array}{l}\text { Journal } \\
\text { Abbreviation }\end{array}$ & Publisher & Year & Country & Citation \\
\hline 26 & {$[106]$} & Veneri et al. & $\begin{array}{l}\text { a bidirectional } \\
\text { DC-DC converter }\end{array}$ & $\begin{array}{l}\text { Hybrid Energy } \\
\text { Storage System }\end{array}$ & $\begin{array}{c}\text { Dynamic test bench; SC; EV; EMS; } \\
\text { HESS; ZEBRA batteries }\end{array}$ & Article & APEND & Elsevier Ltd. & 2018 & Italy & 47 \\
\hline 27 & [107] & Pires et al. & $\begin{array}{l}\text { two new non-isolated } \\
\text { DC-DC converters }\end{array}$ & Fuel Cell Storage & $\begin{array}{l}\text { FCEV; low-voltage stress; non-isolated } \\
\text { converter; Quadratic Boost and Cuk } \\
\text { converters; single-switch; wide } \\
\text { voltage-gain }\end{array}$ & Article & ITVTA & IEEE & 2019 & Portugal & 41 \\
\hline 28 & {$[108]$} & Zhang et al. & DC-DC converter & $\begin{array}{l}\text { Hybrid Energy } \\
\text { Storage System }\end{array}$ & Battery life; EV; EM; HESS & Article & ITPEE & IEEE & 2020 & China & 40 \\
\hline 29 & [109] & Jiang et al. & bidirectional converter & Battery Storage & $\begin{array}{l}\text { BDC; dynamic characteristic; harmonic } \\
\text { analysis; single-stage; V2G }\end{array}$ & Article & Energies & MDPI AG & 2014 & China & 39 \\
\hline 30 & [110] & Wang et al. & $\begin{array}{l}\text { a bidirectional (Bi) } \\
\text { DC-DC converter }\end{array}$ & $\begin{array}{l}\text { Hybrid Energy Storage } \\
\text { System }\end{array}$ & $\begin{array}{l}\text { DC-DC; efficiency model; EV; energy } \\
\text { loss; power dissipation; topology }\end{array}$ & Article & APEND & Elsevier Ltd. & 2016 & China & 38 \\
\hline 31 & {$[111]$} & Muttaqi et al. & $\begin{array}{l}\text { power-electronic } \\
\text { converters }\end{array}$ & $\begin{array}{l}\text { Hybrid Energy } \\
\text { Storage System }\end{array}$ & $\begin{array}{l}\text { EV; ES; future power grids; magnetic } \\
\text { bus; RES; SC }\end{array}$ & Article & ITASE & IEEE & 2019 & Australia & 32 \\
\hline 32 & [112] & Wen et al. & $\begin{array}{l}\text { a hybrid-mode } \\
\text { two-phase interleaved } \\
\text { boost converter }\end{array}$ & Fuel Cell Storage & $\begin{array}{c}\text { Continuous conduction mode; } \\
\text { discontinuous conduction mode; } \\
\text { efficiency; FCEV; input current ripple; } \\
\text { interleaved boost converter; output } \\
\text { voltage ripple }\end{array}$ & Article & ECMAD & Elsevier Ltd. & 2016 & China & 32 \\
\hline 33 & [113] & $\begin{array}{l}\text { Kandasamy } \\
\text { et al. }\end{array}$ & $\begin{array}{l}\text { cascaded H-bridge } \\
(\mathrm{CHB}) \text { converter }\end{array}$ & Battery Storage & $\begin{array}{l}\text { Amphibious vehicles; BMS; bridge } \\
\text { circuits; charging (batteries); EB; EV; } \\
\text { fault tolerance }\end{array}$ & Article & IETPE & IET & 2015 & Singapore & 32 \\
\hline 34 & [114] & $\begin{array}{l}\text { Mukherjee } \\
\text { et al. }\end{array}$ & $\begin{array}{l}\text { dc-side modular } \\
\text { multilevel converter }\end{array}$ & $\begin{array}{l}\text { Hybrid Energy } \\
\text { Storage System }\end{array}$ & $\begin{array}{l}\text { Boost-multilevel buck converter; } \\
\text { comparison; HESS; multiple battery } \\
\text { types; second-life battery energy } \\
\text { storage systems }\end{array}$ & Article & IJESTP & IEEE & 2016 & $\begin{array}{l}\text { United } \\
\text { King- } \\
\text { dom }\end{array}$ & 30 \\
\hline 35 & [115] & Wang et al. & boost converter & $\begin{array}{l}\text { Hybrid Energy } \\
\text { Storage System }\end{array}$ & $\begin{array}{l}\text { Adaptive sliding-mode control; boost } \\
\text { converter; EV; EM; HESS; SC }\end{array}$ & Article & ECMAD & Elsevier Ltd. & 2017 & China & 27 \\
\hline 36 & [116] & Amjadi et al. & $\begin{array}{c}\text { a hybrid } \\
\text { switched-capacitor } \\
\text { bidirectional } \\
\text { DC-DC converter }\end{array}$ & $\begin{array}{l}\text { Hybrid Energy } \\
\text { Storage System }\end{array}$ & BS; CS; efficiency; EV; PE; UC & Article & ITSG & IEEE & 2014 & Canada & 27 \\
\hline 37 & [117] & Zhang et al. & $\begin{array}{c}\text { bidirectional } \\
\text { DC-DC converter }\end{array}$ & $\begin{array}{l}\text { Hybrid Energy } \\
\text { Storage System }\end{array}$ & $\begin{array}{l}\text { Coupled inductor; DC-DC converter; } \\
\text { HESS; soft-switching; ZVS }\end{array}$ & Article & ITIED & IEEE & 2018 & China & 26 \\
\hline
\end{tabular}


Table 3. Cont.

\begin{tabular}{|c|c|c|c|c|c|c|c|c|c|c|c|}
\hline Rank & Ref. & Authors & Converter Type & Energy Storage Type & Author Keywords & Article Type & $\begin{array}{c}\text { Journal } \\
\text { Abbreviation }\end{array}$ & Publisher & Year & Country & Citation \\
\hline 38 & [118] & Badawy et al. & $\begin{array}{l}\text { an interfacing DC-DC } \\
\text { converter }\end{array}$ & $\begin{array}{c}\text { Hybrid Energy Storage } \\
\text { System }\end{array}$ & $\begin{array}{l}\text { Battery; DC-DC converter; EV; HESS; } \\
\text { interior permanent magnet; inverter; } \\
\text { motor control; permanent magnet } \\
\text { motor; UC }\end{array}$ & Article & ITIAC & IEEE & 2017 & $\begin{array}{l}\text { United } \\
\text { States }\end{array}$ & 26 \\
\hline 39 & [119] & $\begin{array}{l}\text { Michalczuk } \\
\text { et al. }\end{array}$ & $\begin{array}{c}\text { two DC-DC } \\
\text { interleaved converters }\end{array}$ & $\begin{array}{c}\text { Hybrid Energy Storage } \\
\text { System }\end{array}$ & EV; FL; HESS; UC & Article & CODUD & $\begin{array}{l}\text { Emerald } \\
\text { Group } \\
\text { Publishing } \\
\text { Ltd. }\end{array}$ & 2015 & Poland & 26 \\
\hline 41 & [121] & Yang et al. & $\begin{array}{l}\text { two bidirectional } \\
\text { DC-DC converters }\end{array}$ & $\begin{array}{c}\text { Hybrid Energy Storage } \\
\text { System }\end{array}$ & $\begin{array}{l}\text { Battery/supercapacitor HESS; EV; } \\
\text { perturbation observer; robust } \\
\text { fractional-order sliding-mode control }\end{array}$ & Article & JPSOD & Elsevier B.V. & 2020 & China & 25 \\
\hline 42 & [122] & Amjadi et al. & $\begin{array}{c}\text { a hybrid } \\
\text { switched-capacitor } \\
\text { bidirectional DC-DC } \\
\text { converter }\end{array}$ & $\begin{array}{l}\text { Hybrid Energy Storage } \\
\text { System }\end{array}$ & BS; CS; efficiency; EV; PE; UC & Article & ITVTA & IEEE & 2011 & Canada & 24 \\
\hline 44 & [73] & Sorlei et al. & DC-DC converters & Fuel Cell Storage & $\begin{array}{l}\text { DC-DC converter topologies; EMS; } \\
\text { FCEV; global optimization; real-time } \\
\text { optimization; rule-based }\end{array}$ & Review & Energies & MDPI AG & 2021 & Romania & 22 \\
\hline 45 & [124] & Khan et al. & $\begin{array}{l}\text { an isolated hybrid } \\
\text { multi-port converter }\end{array}$ & Battery Storage & $\begin{array}{l}\text { BC; clean energy; EV; isolation; } \\
\text { micro-grid; multi-port converter }\end{array}$ & Article & ITASE & IEEE & 2019 & Australia & 22 \\
\hline 46 & [125] & Capasso et al. & $\begin{array}{l}\text { a DC-DC bidirectional } \\
\text { power converter }\end{array}$ & $\begin{array}{c}\text { Hybrid Energy Storage } \\
\text { System }\end{array}$ & $\begin{array}{c}\text { Dynamic test bench; EMS; HESS; } \\
\text { modelling of electric double layer } \\
\text { capacitors }\end{array}$ & Article & APEND & Elsevier Ltd. & 2018 & Italy & 22 \\
\hline 47 & [126] & Zhang et al. & DC-DC converter & $\begin{array}{c}\text { Hybrid Energy Storage } \\
\text { System }\end{array}$ & $\begin{array}{c}\text { Controllers; cost-benefit analysis; cost } \\
\text { effectiveness; DC-DC converters; EB; } \\
\text { EE; EM; ES; HESS }\end{array}$ & Article & JCSE & $\begin{array}{l}\text { Hindawi } \\
\text { Publishing } \\
\text { Corporation }\end{array}$ & 2016 & China & 22 \\
\hline
\end{tabular}


Table 3. Cont.

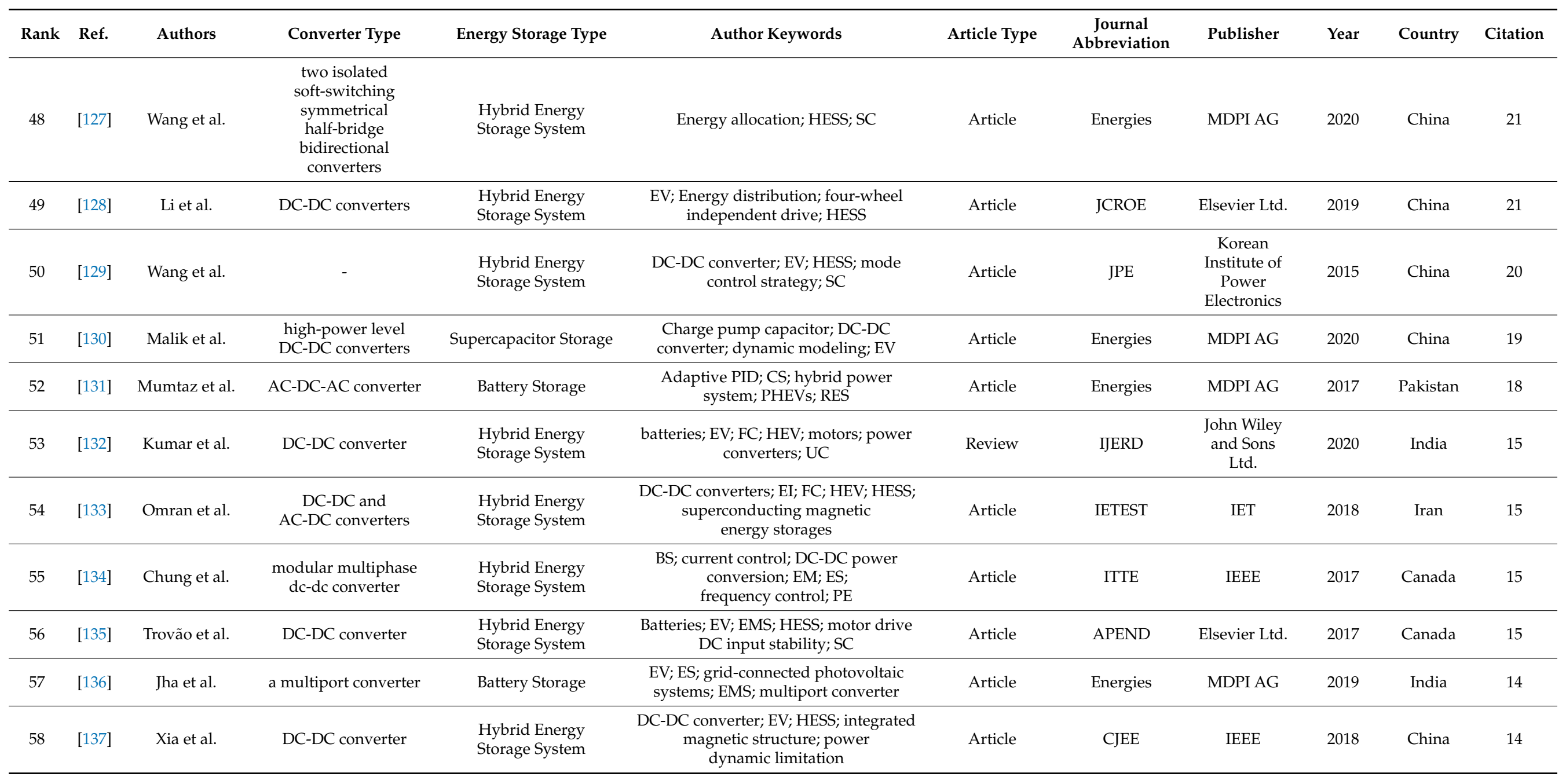


Table 3. Cont.

\begin{tabular}{|c|c|c|c|c|c|c|c|c|c|c|c|}
\hline Rank & Ref. & Authors & Converter Type & Energy Storage Type & Author Keywords & Article Type & $\begin{array}{c}\text { Journal } \\
\text { Abbreviation }\end{array}$ & Publisher & Year & Country & Citation \\
\hline 59 & {$[76]$} & Zhang et al. & DC-DC converter & $\begin{array}{l}\text { Hybrid Energy } \\
\text { Storage System }\end{array}$ & $\begin{array}{l}\text { Case study; DC-DC converter; EV; EM; } \\
\text { HESS; optimal sizing; smart grid }\end{array}$ & Review & RSERF & Elsevier Ltd. & 2021 & China & 13 \\
\hline 60 & [138] & Becker et al. & $\begin{array}{c}\text { two individual } \\
\text { DC-DC converters }\end{array}$ & Battery Storage & BMS; EV; EMS; Li-ion battery system & Article & JEET & $\begin{array}{l}\text { Korea } \\
\text { Science }\end{array}$ & 2013 & Germany & 11 \\
\hline 61 & [139] & Wang et al. & $\begin{array}{l}\text { a new bidirectional } \\
\text { three-level cascaded } \\
\text { (BTLC) converter }\end{array}$ & $\begin{array}{l}\text { Hybrid Energy } \\
\text { Storage System }\end{array}$ & $\begin{array}{l}\text { Deadbeat control; EV; HESS; three-level } \\
\text { cascaded converter }\end{array}$ & Article & ITTE & IEEE & 2019 & China & 10 \\
\hline 63 & [141] & Wang et al. & $\begin{array}{c}\text { two associated } \\
\text { DC-DC converters }\end{array}$ & $\begin{array}{l}\text { Hybrid Energy } \\
\text { Storage System }\end{array}$ & $\begin{array}{l}\text { EM; HESS; PHEV; temperature } \\
\text { uncertainty; Wavelet transform }\end{array}$ & Article & APEND & Elsevier Ltd. & 2019 & China & 9 \\
\hline 64 & [142] & Liu et al. & $\begin{array}{c}\text { bidirectional } \\
\text { DC-DC converter }\end{array}$ & Battery Storage & $\begin{array}{l}\text { BS; EV; energy internet; energy router; } \\
\text { microgrid; PV }\end{array}$ & Article & Energies & MDPI AG & 2019 & China & 8 \\
\hline 65 & [143] & Cheng et al. & $\begin{array}{l}\text { a family of } \\
\text { non-isolated DC-DC } \\
\text { three-port converters }\end{array}$ & $\begin{array}{l}\text { Hybrid Energy } \\
\text { Storage System }\end{array}$ & $\begin{array}{c}\text { Bi-directional loads; dual-input } \\
\text { single-output; ES; RES; single-input } \\
\text { dual-output; single-input single-output; } \\
\text { three-port converter }\end{array}$ & Article & JPE & $\begin{array}{l}\text { Korean } \\
\text { Institute of } \\
\text { Power } \\
\text { Electronics }\end{array}$ & 2017 & Australia & 8 \\
\hline 66 & [144] & $\begin{array}{l}\text { Moradisizkoohi } \\
\text { et al. }\end{array}$ & $\begin{array}{l}\text { a double-input } \\
\text { three-level converter }\end{array}$ & $\begin{array}{l}\text { Hybrid Energy } \\
\text { Storage System }\end{array}$ & $\begin{array}{l}\text { Double-input converter; multilevel } \\
\text { converter; RES; wide } \\
\text { bandgap semiconductors }\end{array}$ & Article & ITIAC & IEEE & 2019 & $\begin{array}{l}\text { United } \\
\text { States }\end{array}$ & 6 \\
\hline 67 & [145] & Bae et al. & $\begin{array}{l}\text { a bidirectional } \\
\text { DC-DC converter }\end{array}$ & $\begin{array}{l}\text { Hybrid Energy } \\
\text { Storage System }\end{array}$ & $\begin{array}{l}\text { HESS; lead-acid battery; lithium-ion } \\
\text { battery; HEV; state-of-charge }\end{array}$ & Article & JEET & $\begin{array}{l}\text { Korean } \\
\text { Institute of } \\
\text { Electrical } \\
\text { Engineers }\end{array}$ & 2018 & $\begin{array}{l}\text { South } \\
\text { Korea }\end{array}$ & 5 \\
\hline 68 & [74] & $\begin{array}{l}\text { Moradisizkoohi } \\
\text { et al. }\end{array}$ & $\begin{array}{l}\text { a voltage-quadrupler } \\
\text { interleaved } \\
\text { bidirectional } \\
\text { dc-dc converter }\end{array}$ & $\begin{array}{l}\text { Hybrid Energy } \\
\text { Storage System }\end{array}$ & $\begin{array}{l}\text { Bidirectional DC-DC converter; EV; } \\
\text { interleaved converter; non-isolated } \\
\text { converter; wide-bandgap devices }\end{array}$ & Article & ITIED & IEEE & 2021 & $\begin{array}{l}\text { United } \\
\text { States }\end{array}$ & 4 \\
\hline 69 & [146] & Bindu et al. & - & Supercapacitor Storage & $\begin{array}{l}\text { EV; induction motor; LFP battery; } \\
\text { multi-input BDC; power flow; three } \\
\text { phase inverter; UC }\end{array}$ & Article & IJR & $\begin{array}{l}\text { Taylor and } \\
\text { Francis Ltd. }\end{array}$ & 2020 & India & 4 \\
\hline 70 & [147] & Wang et al. & $\begin{array}{l}\text { a bidirectional } \\
\text { non-isolated } \\
\text { DC-DC converter }\end{array}$ & $\begin{array}{l}\text { Hybrid Energy } \\
\text { Storage System }\end{array}$ & $\begin{array}{l}\text { BDC; HESS; low-voltage stress; } \\
\text { wide-voltage gain range }\end{array}$ & Article & JPE & Springer & 2020 & China & 4 \\
\hline
\end{tabular}


Table 3. Cont.

\begin{tabular}{|c|c|c|c|c|c|c|c|c|c|c|c|}
\hline Rank & Ref. & Authors & Converter Type & Energy Storage Type & Author Keywords & Article Type & $\begin{array}{c}\text { Journal } \\
\text { Abbreviation }\end{array}$ & Publisher & Year & Country & Citation \\
\hline 71 & [148] & Bai et al. & - & $\begin{array}{l}\text { Hybrid Energy } \\
\text { Storage System }\end{array}$ & $\begin{array}{c}\text { EV; Hocontroller; HESS; regenerative } \\
\text { braking; SC }\end{array}$ & Article & IJAT & $\begin{array}{l}\text { Korean } \\
\text { Society of } \\
\text { Automotive } \\
\text { Engineers }\end{array}$ & 2019 & China & 4 \\
\hline 72 & [149] & Vidhya et al. & $\begin{array}{c}\text { an efficient } \\
\text { bi-directional converter }\end{array}$ & $\begin{array}{l}\text { Hybrid Energy } \\
\text { Storage System }\end{array}$ & $\begin{array}{l}\text { BDC; EMS; HESS; Indian driving cycle; } \\
\text { light electric vehicle }\end{array}$ & Article & MEACB & $\begin{array}{l}\text { SAGE } \\
\text { Publications } \\
\text { Ltd. }\end{array}$ & 2019 & India & 4 \\
\hline 73 & {$[75]$} & Daya et al. & $\begin{array}{l}\text { a novel non-isolated } \\
\text { multi-input DC-DC } \\
\text { boost converter }\end{array}$ & Battery Storage & $\begin{array}{l}\text { charging; controller; DC-DC boost } \\
\text { converter; discharging; EV; small } \\
\text { signal model }\end{array}$ & Article & IJEEPS & $\begin{array}{l}\text { De Gruyter } \\
\text { Open Ltd. }\end{array}$ & 2021 & India & 3 \\
\hline 74 & [150] & de Melo et al. & $\begin{array}{l}\text { an interleaved } \\
\text { bidirectional } \\
\text { DC-DC converter }\end{array}$ & Supercapacitor Storage & $\begin{array}{l}\text { Bidirectional power flow; DC-DC } \\
\text { converters; EV; interleaving; SC }\end{array}$ & Article & EENGF & Springer & 2020 & Brazil & 3 \\
\hline 75 & [151] & $\begin{array}{l}\text { Veerendra } \\
\text { et al. }\end{array}$ & 4-level converter & $\begin{array}{l}\text { Hybrid Energy } \\
\text { Storage System }\end{array}$ & $\begin{array}{l}\text { fuel cell and battery; HEV; multilevel } \\
\text { converter; switched reluctance motor }\end{array}$ & Article & IJAED & $\begin{array}{l}\text { Taylor and } \\
\text { Francis Ltd. }\end{array}$ & 2020 & Malaysia & 3 \\
\hline 76 & [152] & Joseph et al. & $\begin{array}{l}\text { a hybrid forward-boost } \\
\text { converter }\end{array}$ & $\begin{array}{l}\text { Hybrid Energy } \\
\text { Storage System }\end{array}$ & $\begin{array}{l}\text { Charging (batteries); ES; RES; charging } \\
\text { efficiency; electric vehicle charging; ESS; } \\
\text { DC-DC converters }\end{array}$ & Article & IETPE & IET & 2019 & India & 3 \\
\hline 78 & [154] & Jiang et al. & $\begin{array}{l}\text { a bidirectional } \\
\text { DC-DC converter }\end{array}$ & $\begin{array}{l}\text { Hybrid Energy } \\
\text { Storage System }\end{array}$ & $\begin{array}{l}\text { Bidirectional; DC-DC converter; HESS; } \\
\text { maximum efficiency; peak-valley } \\
\text { control; variable frequency control; zero } \\
\text { voltage switching }\end{array}$ & Article & IJPE & $\begin{array}{l}\text { Inderscience } \\
\text { Publishers }\end{array}$ & 2014 & China & 3 \\
\hline 79 & [155] & Rahman et al. & DC-DC converters & $\begin{array}{l}\text { Hybrid Energy } \\
\text { Storage System }\end{array}$ & $\begin{array}{l}\text { Fuzzy based EM; HESS; hydrogen fuel } \\
\text { consumption; super twisting sliding } \\
\text { mode control }\end{array}$ & Article & JES & Elsevier Ltd. & 2021 & Pakistan & 2 \\
\hline 80 & [156] & Suresh et al. & four-port converter & Battery Storage & $\begin{array}{l}\text { BS; BDC; EV; multi-port converter; } \\
\text { regenerative charging }\end{array}$ & Article & IEEE Access & IEEE & 2021 & India & 2 \\
\hline 81 & [157] & Tseng et al. & $\begin{array}{l}\text { a full-bridge } \\
\text { DC-DC converter }\end{array}$ & $\begin{array}{l}\text { Hybrid Energy } \\
\text { Storage System }\end{array}$ & $\begin{array}{l}\text { CS; DC-DC converters; LVP; light rail } \\
\text { transit; SC; charging current; HESS }\end{array}$ & Article & IETPE & IET & 2020 & Taiwan & 2 \\
\hline 82 & [158] & Lai et al. & $\begin{array}{l}\text { a patented } \\
\text { bidirectional power } \\
\text { converter }\end{array}$ & $\begin{array}{l}\text { Hybrid Energy } \\
\text { Storage System }\end{array}$ & $\begin{array}{l}\text { Battery/ultracapacitor; BDC; } \\
\text { dual-energy; EV }\end{array}$ & Article & Energies & MDPI AG & 2020 & Taiwan & 2 \\
\hline
\end{tabular}


Table 3. Cont.

\begin{tabular}{|c|c|c|c|c|c|c|c|c|c|c|c|}
\hline Rank & Ref. & Authors & Converter Type & Energy Storage Type & Author Keywords & Article Type & $\begin{array}{c}\text { Journal } \\
\text { Abbreviation }\end{array}$ & Publisher & Year & Country & Citation \\
\hline 83 & [159] & Deng et al. & $\begin{array}{l}\text { a novel multiphase } \\
\text { bidirectional } \\
\text { DC-DC converter }\end{array}$ & Fuel Cell Storage & $\begin{array}{l}\text { BDC; EM; FCEV; real-time } \\
\text { digital simulator }\end{array}$ & Article & JPE & $\begin{array}{c}\text { Korean } \\
\text { Institute of } \\
\text { Power } \\
\text { Electronics }\end{array}$ & 2011 & $\begin{array}{l}\text { United } \\
\text { States }\end{array}$ & 2 \\
\hline 84 & [78] & $\begin{array}{l}\text { Chelladurai } \\
\text { et al. }\end{array}$ & $\begin{array}{l}\text { an interval type-2 } \\
\text { fuzzy logic-controlled } \\
\text { shunt converter }\end{array}$ & Battery Storage & $\begin{array}{l}\text { Battery charger; EVs; FLC; power } \\
\text { quality; total harmonic distortion }\end{array}$ & Article & ITII & IEEE & 2021 & India & 1 \\
\hline 85 & [160] & Lipu et al. & $\begin{array}{c}\text { power } \\
\text { electronics converters }\end{array}$ & Battery Storage & $\begin{array}{l}\text { BSS; DC-DC converter; EV; intelligent } \\
\text { controller; meta-heuristic optimization; } \\
\text { modulation techniques }\end{array}$ & Review & Electronics & MDPI AG & 2021 & Malaysia & 1 \\
\hline 86 & [161] & Eroğlu et al. & Multilevel converters & Battery Storage & $\begin{array}{l}\text { Automotive batteries; BMS; charging } \\
\text { (batteries); EPTN; PC; RES; V2G; EVs }\end{array}$ & Article & IETRPG & $\begin{array}{l}\text { John Wiley } \\
\text { and Sons Inc }\end{array}$ & 2021 & Turkey & 1 \\
\hline 87 & [162] & Tirpude et al. & $\begin{array}{l}\text { a bidirectional } \\
\text { DC-DC converter }\end{array}$ & $\begin{array}{l}\text { Hybrid Energy } \\
\text { Storage System }\end{array}$ & $\begin{array}{c}\text { CS; EV; EMS; HESS; lithium-ion battery; } \\
\text { neural network; Proportional-Integral } \\
\text { (PI) controller; SC }\end{array}$ & Article & IJETER & $\begin{array}{c}\text { World } \\
\text { Academy of } \\
\text { Research in } \\
\text { Science and } \\
\text { Engineering }\end{array}$ & 2020 & India & 1 \\
\hline 88 & [163] & Devi et al. & $\begin{array}{l}\text { a modular multilevel } \\
\text { capacitor } \\
\text { clamped converter }\end{array}$ & $\begin{array}{l}\text { Hybrid Energy } \\
\text { Storage System }\end{array}$ & $\begin{array}{c}\text { CS; EV; failure (mechanical); LVP; } \\
\text { MATLAB; outages; regenerative } \\
\text { braking; SB; SC; BDC }\end{array}$ & Article & IETPE & IET & 2019 & India & 1 \\
\hline 89 & [164] & Kolbasov et al. & $\begin{array}{l}\text { photovoltaic } \\
\text { (PHV) converters }\end{array}$ & $\begin{array}{l}\text { Hybrid Energy } \\
\text { Storage System }\end{array}$ & $\begin{array}{l}\text { Charging infrastructure; CS; EV; } \\
\text { photoelectric converters; solar battery }\end{array}$ & Article & Energies & MDPI AG & 2019 & $\begin{array}{l}\text { Russian } \\
\text { Federa- } \\
\text { tion }\end{array}$ & 1 \\
\hline 90 & [165] & Amjadi et al. & $\begin{array}{l}\text { a 4-quadrant (4-Q) } \\
\text { switched-capacitor } \\
\text { (SC) Luo bidirectional } \\
\text { DC-DC converter }\end{array}$ & $\begin{array}{l}\text { Hybrid Energy } \\
\text { Storage System }\end{array}$ & $\mathrm{BS} ; \mathrm{CS}$; EVs; PE; UC & Article & IJPE & $\begin{array}{l}\text { Inderscience } \\
\text { Publishers }\end{array}$ & 2011 & Canada & 1 \\
\hline 91 & {$[166]$} & Amjadi et al. & $\begin{array}{l}\text { a two-quadrant hybrid } \\
\text { switched capacitor } \\
\text { (SC) converter }\end{array}$ & $\begin{array}{l}\text { Hybrid Energy } \\
\text { Storage System }\end{array}$ & $\begin{array}{c}\text { BS; Capacitors; CS; DC-DC converters; } \\
\text { EV; ES; PE }\end{array}$ & Article & IJEHV & $\begin{array}{l}\text { Inderscience } \\
\text { Publishers }\end{array}$ & 2011 & Canada & 1 \\
\hline 92 & {$[167]$} & Ebrahimi et al. & DC-DC converters & $\begin{array}{l}\text { Hybrid Energy } \\
\text { Storage System }\end{array}$ & $\begin{array}{l}\text { DC-AC converters; EVs; HESS; } \\
\text { multisource inverters; SVM }\end{array}$ & Article & ITPEE & IEEE & 2022 & Canada & 0 \\
\hline
\end{tabular}


Table 3. Cont.

\begin{tabular}{|c|c|c|c|c|c|c|c|c|c|c|c|}
\hline Rank & Ref. & Authors & Converter Type & Energy Storage Type & Author Keywords & Article Type & $\begin{array}{c}\text { Journal } \\
\text { Abbreviation }\end{array}$ & Publisher & Year & Country & Citation \\
\hline 93 & [168] & Suresh et al. & $\begin{array}{l}\text { a modular multi-input } \\
\text { bidirectional DC-DC } \\
\text { buck-boost converter }\end{array}$ & $\begin{array}{l}\text { Hybrid Energy } \\
\text { Storage System }\end{array}$ & BESS; DC-DC multi-port converter; UC & Article & JASE & $\begin{array}{l}\text { Tamkang } \\
\text { University }\end{array}$ & 2022 & India & 0 \\
\hline 94 & [169] & $\begin{array}{l}\text { de } \\
\text { Oliveira-Assis } \\
\text { et al. }\end{array}$ & $\begin{array}{c}\text { integrates } \\
\text { Z-source converters }\end{array}$ & $\begin{array}{l}\text { Hybrid Energy } \\
\text { Storage System }\end{array}$ & $\begin{array}{l}\text { EV; EMS; hydrogen system; } \\
\text { microgrid; PC }\end{array}$ & Article & ECMAD & Elsevier Ltd. & 2021 & Spain & 0 \\
\hline 95 & {$[80]$} & Shi et al. & $\begin{array}{l}\text { an additional cascaded } \\
\text { DC-DC converter }\end{array}$ & $\begin{array}{l}\text { Hybrid Energy Storage } \\
\text { System }\end{array}$ & $\begin{array}{l}\text { Dual-inverter; EV; EM; HESS; } \\
\text { open-winding machine }\end{array}$ & Article & ITIED & IEEE & 2021 & Canada & 0 \\
\hline 96 & {$[79]$} & Raman et al. & $\begin{array}{c}\text { interleaved } \\
\text { bi-directional converter }\end{array}$ & $\begin{array}{l}\text { Hybrid Energy } \\
\text { Storage System }\end{array}$ & $\begin{array}{l}\text { Configurable EV; distributed ES; EV; } \\
\text { HESS; interleaved converter; SC }\end{array}$ & Article & Energies & MDPI & 2021 & $\begin{array}{l}\text { Hong } \\
\text { Kong }\end{array}$ & 0 \\
\hline 97 & {$[77]$} & Fang et al. & $\begin{array}{l}\text { interleaved } \\
\text { quasi-resonant converters }\end{array}$ & Battery Storage & $\begin{array}{l}\text { High conversion ratio; onboard ESS; } \\
\text { resonant converter; small characteristic } \\
\text { impedance; zero current switching }\end{array}$ & Article & ITVTA & IEEE & 2021 & China & 0 \\
\hline 98 & [170] & Shen et al. & $\begin{array}{c}\text { a multi-port } \\
\text { DC-DC converter }\end{array}$ & $\begin{array}{l}\text { Hybrid Energy } \\
\text { Storage System }\end{array}$ & battery life; EMS; FLC; HESS & Article & ITEEE & $\begin{array}{l}\text { John Wiley } \\
\text { and Sons Inc }\end{array}$ & 2021 & China & 0 \\
\hline 99 & {$[71]$} & $\begin{array}{l}\text { Katnapally } \\
\text { et al. }\end{array}$ & $\begin{array}{l}\text { multiple-input } \\
\text { bidirectional } \\
\text { DC-DC converter }\end{array}$ & $\begin{array}{l}\text { Hybrid Energy } \\
\text { Storage System }\end{array}$ & $\begin{array}{l}\text { BDC; EV; HESS; model } \\
\text { predictive control }\end{array}$ & Article & ICTAC & $\begin{array}{l}\text { John Wiley } \\
\text { and Sons } \\
\text { Ltd. }\end{array}$ & 2021 & India & 0 \\
\hline 100 & {$[72]$} & Fang et al. & grid-tied converters & Battery Storage & $\begin{array}{l}\text { Active filters; ancillary services; } \\
\text { batteries; capacitors; droop control; grid } \\
\text { forming; phase locked loops; PC }\end{array}$ & Article & IJESTP & IEEE & 2021 & $\begin{array}{l}\text { United } \\
\text { States }\end{array}$ & 0 \\
\hline
\end{tabular}

ES = Energy Storage, EV = Electric Vehicles, SB = Secondary Batteries, HV = Hybrid Vehicles, HESS = Hybrid Energy Storage Systems, EM = Energy Management, SC = Supercapacitor $\mathrm{PC}=$ Power Converters, EMS = Energy Management Systems, EI = Electric Inverters, LVP = Land Vehicle Propulsion, FC $=$ Fuel Cells, EB $=$ Electric Batteries, FS = Fuel Storage, $\mathrm{SM}=$ Storage Management, BMS = Battery Management Systems, CS = Capacitor Storage, ELF = Electric Load Flow, EPTN = Electric Power Transmission Networks, UC = Ultracapacitors EMC = Electric Machine Control, HVDCPT = HVDC Power Transmission, VA = Vehicle Applications, BS = Battery Storage, RES = Renewable Energy Resources, BDC = Bidirectional

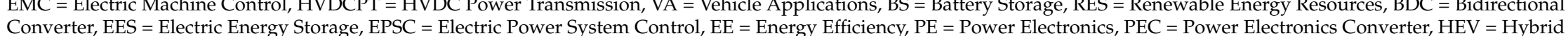
Electric Vehicle, RB = Regenerative Braking, SC = Solar Cells, V2G = Vehicle-to-grid, FL = Fuzzy Logic, PHEV = Plug-in Hybrid Vehicles, TM = Traction Motors, VC = Voltage Control, BP $=$ Battery Pack, MIMO = Multiple-input-multiple-output, MMC = modular multilevel converter, SRM = switched reluctance motor 
The most referenced publication in the field of energy storage management in electric vehicles is "A New Battery/Ultracapacitor Hybrid Energy Storage System for Electric, Hybrid, and Plug-In Hybrid Electric Vehicles," along with 809 citations, which is generated by Cao et al. and published in the IEEE Transactions on Power Electronics journal in 2012 [81]. In this manuscript, the authors presented a novel battery and supercapacitorbased hybrid energy storage system (HESS) for electric drive cars, such as plug-in hybrid electric vehicles and hybrid electric vehicles. Experimental and simulation outcomes also validate the proposed HESS in EV applications. According to the citation, "Multi-Objective Optimization of a Semi-Active Battery/Supercapacitor Energy Storage System for Electric Vehicles" is an original article by Song et al. that evaluates a semi-active battery and ultracapacitor-based hybrid energy storage system for EV applications. To incorporate the supercapacitors and batteries in the proposed HESS, a considerably smaller unidirectional dc-dc converter was used, resulting in increased HESS efficiency and lower system costs [82]. In 2014, the manuscript was published in the "Applied Energy" journal, and it received 193 citations. "A Hybrid Cascaded Multilevel Converter for Battery Energy Management Applied in Electric Vehicles" [83] was the third most referenced paper published in the "IEEE Transactions on Power Electronics", publication in 2014. Zheng et al. produced the manuscripts, which obtained 164 citations. This research work suggests a hybrid cascaded multilayer converter for EV, which includes both battery energy management and motor drives. Each battery cell in the proposed topology may be connected to the circuit or bypassed by a half-bridge converter. According to Table 3, the first ten articles are the original research work.

\subsection{Distribution of the 100 Manuscripts Chosen from 2010 to 2021}

The allocation of the selected 100 most relevant manuscripts in PEC integrated ESM in EV applications from 2010 to 2021 is illustrated in Figure 4. The number of articles produced between the years 2010 and 2013 was around four. According to Figure 4, the article publication is highest in 2021 (17 research articles), while 2012 delivers the lowest research publication (two research articles) rate, respectively. With 13 manuscripts each, the number of articles published in 2017 and 2020 was the same. Generally, the manuscripts publication trend from 2012 to 2021 indicates an upward trend along with several fluctuations. The years 2014 demonstrate the same number of articles, indicating six.

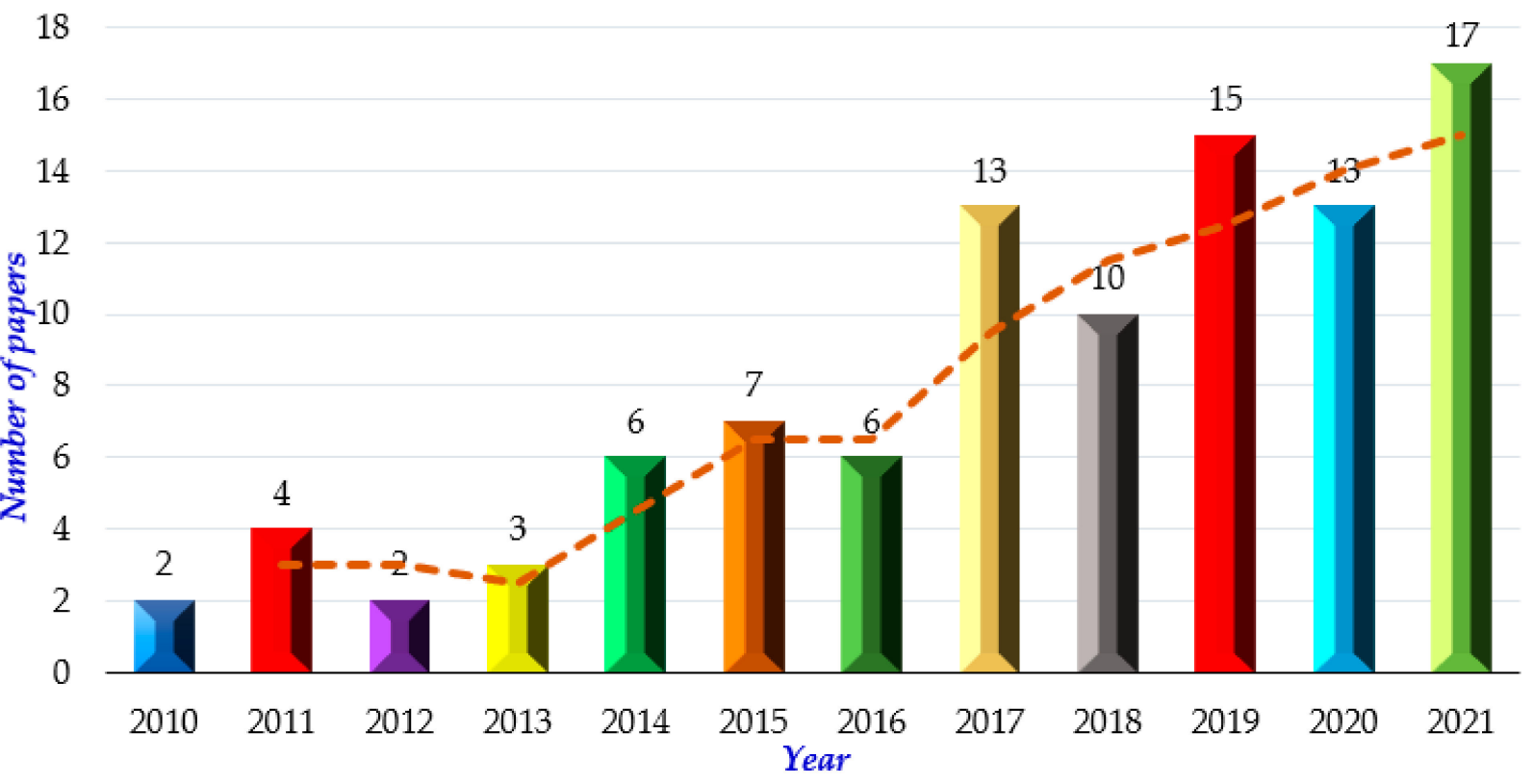

Figure 4. Distribution of selected research articles from 2010 to 2021. 


\subsection{Co-Occurrence Keyword Analysis}

Table 4 and Figure 5 denote the co-occurrence keyword analysis obtained from the 100 most relevant publications using the Scopus database. Figure 5 depicts the connective network of highly frequent keywords produced in the VOSviewer program. The impact of appropriate keywords determines the volume of the circle and label, but the connecting line between the keywords is portrayed as a conjunctive connection. Various colors are used to distinguish different clusters depending on the area of expertise. The power converters, plug-in electric vehicle, electric machine control, electric power transmission network, HVDC power control, voltage control, vehicle to grid, electric load flow, cascaded multilevel converter, and matrix converter are in the red cluster that represents the power converter-related factors. The blue cluster represents the optimization techniques and powers electronics converters such as fuzzy logic control, DC-DC converter, supercapacitor, interleaved converter, multilevel converter, multiport converter, automotive batteries, optimization, and multi-objective optimization for smooth power transition. Energy efficiency, energy management, voltage regulations, fuel cells, hybrid energy storage management, electric batteries are clustered by green color. Finally, electric vehicles, capacitor storage, ultracapacitors, controllers, control strategies, buck converter, switched capacitors, and power electronics, are directly connected to electric vehicles and presented by a yellow cluster.

Table 4 shows the top 15 general keywords from the selected database that were used in multiple papers between 2010 and 2021. Based on Table 4, the existing literature gaps may be identified, and insight into the current study area in the subject can be gained. "DCDC Converters", "Energy Storage", and "Electric Vehicles" are the three most prevalent terms in Table 4. The values for "DC-DC Converters" and "Energy Storage" are 71 and 60, respectively, while "Electric Vehicles" has a figure of 45. "DC-DC Converters", "Energy Storage", and "Energy Management" have also been the most popular terms in the recent two years, reflecting the growing interest in energy storage management in EV application. The exact distribution of keywords and the graphical depiction of Table 4 are depicted in Figure 6. As a result of examining the data from the co-occurrence keywords in Figure 6 and Table 4, it can be concluded that:

- Researchers are now concerned not just with energy storage efficiency but also a focus on smooth energy transition.

- There has been a considerable rise in power electronics converter integrated energy storage management in EV applications.

- Scholars are emphasizing power electronic converter controllers and optimizations to achieve stable power transfer between energy storage and motor.

- Nowadays, researchers are also moving towards renewable energy integration with EV applications.

- There has been a tremendous increase in employing bidirectional energy management systems in the vehicle to grid (V2G) and grid to vehicle (G2V) applications.

- Researchers are focusing on the applications of power electronic converters to develop fast-charging stations in EV applications.

\subsection{Analysis of Power Electronic Converter in EVs in Relevant 100 Cited Papers}

Based on the information on Table 3 , the prominent power electronics converters employed in 100 top cited articles have been identified including cascaded multilevel converter [80,83,139], buckboost converter [140,168], neutral point clamp converter [94], matrix converter [96], modular multilevel converter [105,134,163], interleaved boost converter [88,112,119], H-bridge converter [113], switched capacitor bidirectional converter [86,90,98], soft-switching DC-DC converter [117,127], zsource converter [169], quasi-resonant converter [77], and multi-phase DC-DC converter [134,159]. The objectives, advantages, and disadvantages of the aforesaid converters are highlighted in Table 5 . 
Table 4. Top-most 15 keywords from the selected 100 manuscripts between 2010 and 2021 .

\begin{tabular}{|c|c|c|c|c|c|c|c|c|c|c|c|c|c|}
\hline $\begin{array}{c}\text { Top } \\
\text { Keywords }\end{array}$ & 2010 & 2011 & 2012 & 2013 & 2014 & 2015 & 2016 & 2017 & 2018 & 2019 & 2020 & 2021 & Frequency \\
\hline $\begin{array}{c}\text { DC-DC } \\
\text { Converters }\end{array}$ & {$[86,98]$} & $\begin{array}{l}{[122,165,} \\
166,171]\end{array}$ & {$[81,103]$} & {$[85,88,138]$} & $\begin{array}{c}{[82,87,109} \\
116,154]\end{array}$ & $\begin{array}{c}{[84,92,97} \\
113,119,129]\end{array}$ & $\begin{array}{r}{[90,100,110} \\
112,114,126]\end{array}$ & $\begin{array}{c}89,93- \\
95,102,115 \\
118,131,134 \\
135,143,153] \\
\end{array}$ & $\begin{array}{l}{[99,101,106} \\
117,125,133]\end{array}$ & $\begin{array}{c}{[107,128,} \\
139,141,142, \\
144,148,152 \\
163]\end{array}$ & $\begin{array}{c}{[108,121} \\
130,132,140 \\
147,157]\end{array}$ & $\begin{array}{c}{[71,73-} \\
76,78,80,156 \\
170]\end{array}$ & 71 \\
\hline $\begin{array}{l}\text { Energy } \\
\text { Storage }\end{array}$ & [86] & $\begin{array}{c}{[159,165,} \\
166]\end{array}$ & {$[81,103]$} & [85] & $\begin{array}{c}{[82,87,116} \\
154]\end{array}$ & $\begin{array}{c}{[92,97,119} \\
129]\end{array}$ & $\begin{array}{c}{[90,100,110} \\
114,126]\end{array}$ & $\begin{array}{l}{[89,91,94,95,} \\
102,115,134, \\
135,143,153]\end{array}$ & $\begin{array}{c}{[96,99,104} \\
106,117,145]\end{array}$ & $\begin{array}{c}\text { [105,111, } \\
128,139,141 \\
148,152]\end{array}$ & $\begin{array}{c}{[108,121,} \\
127,132,140, \\
147,150]\end{array}$ & [71-80] & 60 \\
\hline $\begin{array}{c}\text { Electric } \\
\text { Vehicles }\end{array}$ & [86] & $\begin{array}{c}{[122,165,} \\
166]\end{array}$ & {$[81,103]$} & {$[85,88,138]$} & $\begin{array}{c}{[83,87,116} \\
154]\end{array}$ & $\begin{array}{c}{[84,92,97} \\
113,119,129]\end{array}$ & {$[100,110]$} & $\begin{array}{r}{[94,102,115} \\
118,135,153]\end{array}$ & {$[106,137]$} & $\begin{array}{c}111,124, \\
128,142,148, \\
163,164]\end{array}$ & $\begin{array}{c}108,121, \\
132,146,150 \\
158]\end{array}$ & {$[78,156,169]$} & 45 \\
\hline $\begin{array}{l}\text { Secondary } \\
\text { Batteries }\end{array}$ & 0 & {$[122,159]$} & {$[81,103]$} & 0 & [82] & $\begin{array}{c}{[92,113,119,} \\
129]\end{array}$ & $\begin{array}{c}{[100,114} \\
126]\end{array}$ & $\begin{array}{c}{[89,95,102,} \\
131,135]\end{array}$ & $\begin{array}{c}{[96,99,104} \\
106,117,133 \\
145]\end{array}$ & $\begin{array}{c}{[105,128,} \\
139,141,142, \\
148,163,164]\end{array}$ & $\begin{array}{l}{[127,140,} \\
151,158]\end{array}$ & $\begin{array}{c}{[71,76,80} \\
155]\end{array}$ & 42 \\
\hline $\begin{array}{c}\text { Energy } \\
\text { Manage- } \\
\text { ment }\end{array}$ & 0 & [159] & 0 & {$[85,88]$} & 0 & {$[92,119]$} & [126] & $\begin{array}{c}{[89,93,102} \\
115,131,134 \\
135]\end{array}$ & $\begin{array}{c}{[104,106,} \\
125]\end{array}$ & $\begin{array}{c}{[136,141} \\
148]\end{array}$ & {$[108,121]$} & $\begin{array}{c}{[73,76,80,} \\
123,169]\end{array}$ & 26 \\
\hline $\begin{array}{c}\text { Charging } \\
\text { (batteries) }\end{array}$ & 0 & 0 & 0 & 0 & [83] & {$[97,113]$} & [100] & $\begin{array}{c}{[91,94,131} \\
134,135]\end{array}$ & [145] & $\begin{array}{c}{[105,124} \\
128,136,148, \\
152,164]\end{array}$ & $\begin{array}{c}{[127,140,} \\
146]\end{array}$ & $\begin{array}{c}{[78,155,161} \\
170]\end{array}$ & 24 \\
\hline Controllers & [98] & $\begin{array}{c}122,165 \\
166]\end{array}$ & 0 & 0 & {$[87,116]$} & 0 & [126] & [89] & 0 & [148] & {$[121,140]$} & {$[78,155]$} & 13 \\
\hline $\begin{array}{c}\text { Electric } \\
\text { Power } \\
\text { Transmis- } \\
\text { sion } \\
\text { Networks }\end{array}$ & 0 & 0 & 0 & 0 & [109] & [97] & [114] & {$[91,131]$} & [96] & $\begin{array}{c}{[111,124} \\
136]\end{array}$ & 0 & {$[72,76,161]$} & 12 \\
\hline $\begin{array}{l}\text { Renewable } \\
\text { Energy } \\
\text { Resources }\end{array}$ & 0 & 0 & 0 & 0 & 0 & [97] & 0 & {$[91,131,143]$} & 0 & $\begin{array}{c}{[124,144,} \\
152]\end{array}$ & [130] & [161] & 10 \\
\hline $\begin{array}{c}\text { Energy } \\
\text { Efficiency }\end{array}$ & 0 & 0 & 0 & 0 & 0 & 0 & {$[110,126]$} & {$[89,153]$} & 0 & $\begin{array}{c}\text { [111,128, } \\
136]\end{array}$ & [150] & [73] & 9 \\
\hline $\begin{array}{c}\text { Power } \\
\text { Electronics }\end{array}$ & [98] & $\begin{array}{c}{[122,165} \\
166]\end{array}$ & {$[81,103]$} & 0 & [116] & 0 & 0 & [134] & 0 & 0 & 0 & [156] & 9 \\
\hline
\end{tabular}


Table 4. Cont.

\begin{tabular}{|c|c|c|c|c|c|c|c|c|c|c|c|c|c|}
\hline $\begin{array}{c}\text { Top } \\
\text { Keywords }\end{array}$ & 2010 & 2011 & 2012 & 2013 & 2014 & 2015 & 2016 & 2017 & 2018 & 2019 & 2020 & 2021 & Frequency \\
\hline Fuzzy Logic & 0 & 0 & 0 & 0 & 0 & [119] & 0 & 0 & 0 & [141] & {$[108,146]$} & {$[78,155,170]$} & 7 \\
\hline $\begin{array}{l}\text { Voltage } \\
\text { Control }\end{array}$ & 0 & 0 & 0 & 0 & [109] & [113] & 0 & 0 & {$[96,99]$} & [142] & [158] & [72] & 7 \\
\hline $\begin{array}{l}\text { Sliding } \\
\text { Mode } \\
\text { Control }\end{array}$ & 0 & 0 & 0 & 0 & 0 & 0 & 0 & $\begin{array}{c}{[89,95,115} \\
153]\end{array}$ & 0 & 0 & [121] & [155] & 6 \\
\hline $\begin{array}{l}\text { Energy } \\
\text { Transfer }\end{array}$ & 0 & 0 & 0 & 0 & 0 & 0 & 0 & [153] & [99] & [152] & [150] & 0 & 4 \\
\hline
\end{tabular}




\section{B Vosviewer}

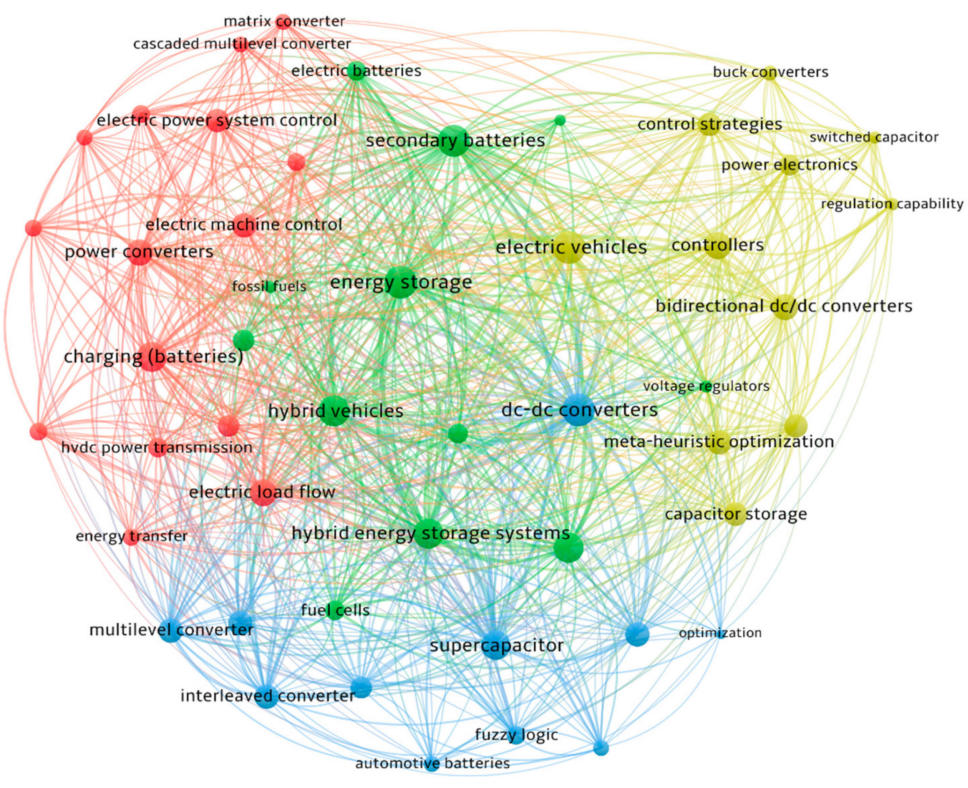

Figure 5. VOSviewer visualization for the analysis of co-occurrence keywords based on the Scopus database.

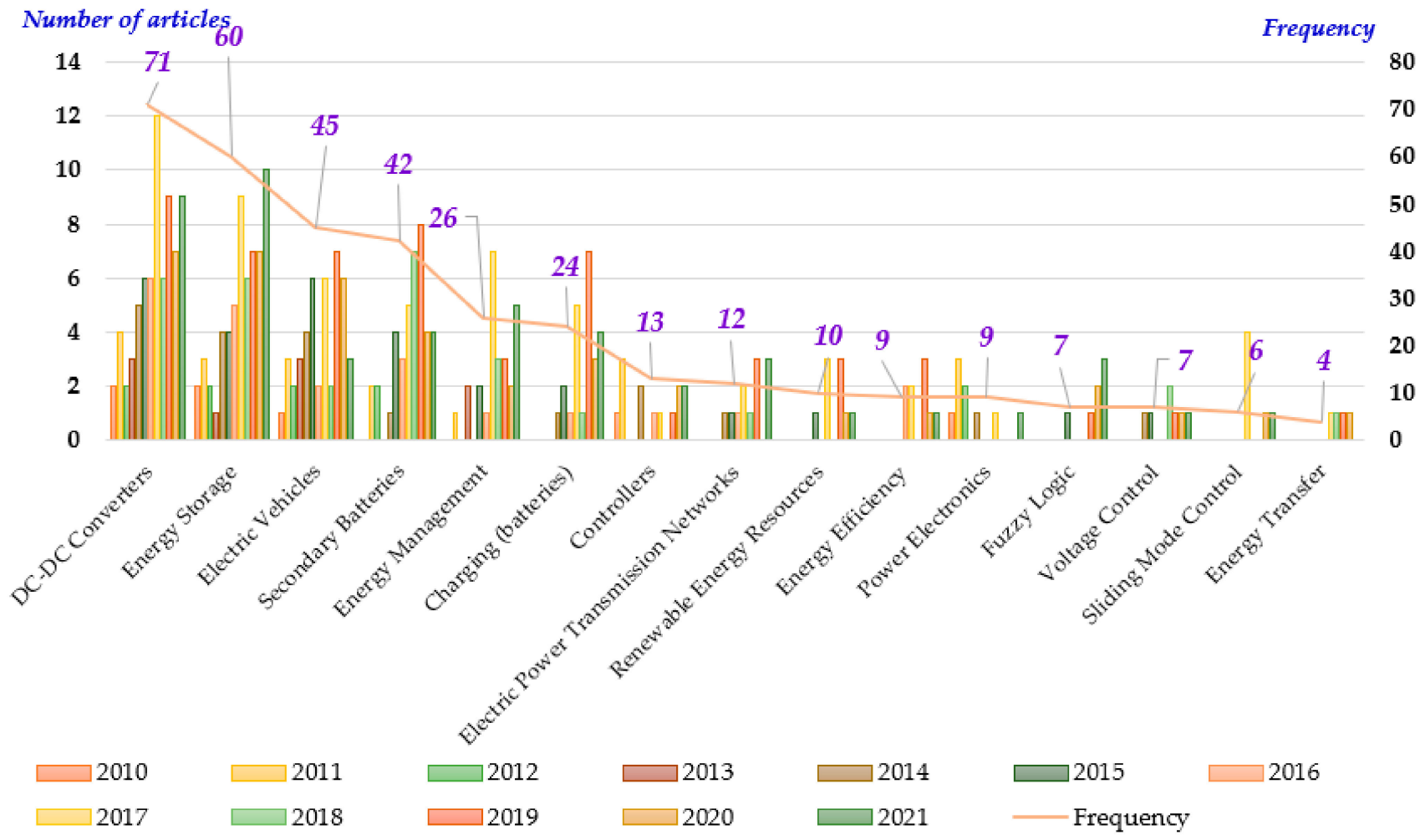

Figure 6. Distribution of topmost 15 keywords over the year 2010 to 2021. 
Table 5. Comparative study of different power electronics converters used in EVs.

\begin{tabular}{|c|c|c|c|c|}
\hline Ref. & $\begin{array}{c}\text { Type of } \\
\text { Converter }\end{array}$ & Target & Advantages & Disadvantages \\
\hline$[80,83,139]$ & $\begin{array}{l}\text { Cascaded } \\
\text { multilevel } \\
\text { converter }\end{array}$ & $\begin{array}{l}\text { - Achieve high-quality } \\
\text { output voltages and } \\
\text { input currents. }\end{array}$ & $\begin{array}{l}\text { - Ability to synthesize } \\
\text { more voltage levels. } \\
\text { Less harmonic distortion } \\
\text { due to the stepped level } \\
\text { voltage output. } \\
\text { - } \quad \text { Less noise generation. } \\
\text { Ease of operation at lower } \\
\text { switching frequencies. }\end{array}$ & $\begin{array}{l}\text { - Higher number of } \\
\text { power semiconductor } \\
\text { switches required. }\end{array}$ \\
\hline$[140,168]$ & $\begin{array}{l}\text { Buck-boost } \\
\text { converter }\end{array}$ & $\begin{array}{l}\text { Deliver a range of } \\
\text { output voltages, } \\
\text { ranging from much } \\
\text { larger than the input } \\
\text { voltage down to } \\
\text { almost zero. }\end{array}$ & $\begin{array}{ll}\text { - } & \text { Provides } \\
\text { efficient solutions. } \\
\text { - Smaller } \\
\text { external components. }\end{array}$ & $\begin{array}{l}\text { - Input current and } \\
\text { charging current of the } \\
\text { output capacitor is } \\
\text { discontinuous, hence, } \\
\text { large filter size and } \\
\text { more EMI issues. } \\
\text { Output is inverted, } \\
\text { which results in } \\
\text { complex sensing and } \\
\text { feedback circuit. }\end{array}$ \\
\hline [94] & $\begin{array}{l}\text { Neutral point } \\
\text { clamp converter }\end{array}$ & $\begin{array}{l}\text { Obtain multilevel } \\
\text { voltage waveform } \\
\text { through a split DC-link, } \\
\text { using multiple sources } \\
\text { and storage through a } \\
\text { single converter. }\end{array}$ & $\begin{array}{l}\text { Superior } \\
\text { - } \quad \text { Leaveform quality. } \\
\text { - } \quad \text { filtering requirements. } \\
\text { Superior blocking } \\
\text { voltage capability. }\end{array}$ & $\begin{array}{l}\text { Requires a greater } \\
\text { number of diodes for } \\
\text { increased levels. } \\
\text { Capacitor voltage } \\
\text { cannot be maintained } \\
\text { as per the selected } \\
\text { switching pattern. }\end{array}$ \\
\hline [96] & $\begin{array}{c}\text { Matrix } \\
\text { converter }\end{array}$ & $\begin{array}{l}\text { Achieve automatic } \\
\text { conversion of power } \\
\text { from AC to AC without } \\
\text { a DC-link capacitor. }\end{array}$ & $\begin{array}{l}\text { A steady AC input source } \\
\text { can be converted directly } \\
\text { into a variable voltage } \\
\text { and variable frequency } \\
\text { output with nine } \\
\text { bidirectional switches. }\end{array}$ & $\begin{array}{l}\text { Limited capacity to } \\
\text { maximize the } \\
\text { output voltage. } \\
\text { Input filter is needed to } \\
\text { reduce the high } \\
\text { switching } \\
\text { frequency harmonics. } \\
\text { Complex, less reliable, } \\
\text { and costly. }\end{array}$ \\
\hline$[105,134,163]$ & $\begin{array}{l}\text { Modular } \\
\text { multilevel } \\
\text { converter }\end{array}$ & $\begin{array}{l}\text { A wide range of } \\
\text { medium and } \\
\text { high-voltage applications. }\end{array}$ & $\begin{array}{l}\text { - Smaller output } \\
\text { harmonic content. } \\
\text { - } \quad \text { Adjustable power factor. } \\
\text { Flexible } \\
\text { control operation. } \\
\text { Weaker the voltage stress } \\
\text { of semiconductor switch } \\
\text { and electromagnetic } \\
\text { disturbances. }\end{array}$ & $\begin{array}{l}\text { - Requires a bulky and } \\
\text { costly isolated } \\
\text { transformer for } \\
\text { each cell. } \\
\text { - } \\
\text { Requires voltage sensor } \\
\text { for each cell. }\end{array}$ \\
\hline
\end{tabular}


Table 5. Cont.

\begin{tabular}{|c|c|c|c|c|}
\hline Ref. & $\begin{array}{c}\text { Type of } \\
\text { Converter }\end{array}$ & Target & Advantages & Disadvantages \\
\hline$[88,112,119]$ & $\begin{array}{c}\text { Interleaved } \\
\text { boost converter }\end{array}$ & $\begin{array}{l}\text { To increase the } \\
\text { efficiency } \\
\text { and reliability. }\end{array}$ & $\begin{array}{ll}\text { - } & \text { Low input current ripple. } \\
\text { - } & \text { High efficiency. } \\
\text { - } & \text { Faster transient response. } \\
\text { - } & \text { Reduced } \\
\text { - } & \text { electromagnetic emission. } \\
\text { Simple control. }\end{array}$ & $\begin{array}{ll}\text { - } & \text { High } \\
\text { - } & \text { High switching losses. } \\
\text { - } & \text { Sensitive to the duty } \\
& \text { cycle variation. }\end{array}$ \\
\hline
\end{tabular}

$\begin{array}{lll}\text { H113] } & \begin{array}{l}\text { H-bridge } \\ \text { converter }\end{array} & \begin{array}{l}\text { Allow DC motors to } \\ \text { run forwards } \\ \text { or backward. }\end{array}\end{array}$

- $\quad$ Lower winding costs.
- $\quad$ Proximity effect losses.
- $\quad$ The half-bridge converter is used in the application of a high voltage requirement.

- It offers slightly more voltage ripple than the half-bridge.

\begin{tabular}{|c|c|c|}
\hline$[86,90,98]$ & $\begin{array}{c}\text { Switched } \\
\text { capacitor } \\
\text { bidirectional }\end{array}$ & $\begin{array}{l}\text { - A high } \\
\text { step-up/step-down } \\
\text { voltage gain. }\end{array}$ \\
\hline
\end{tabular}

$[117,127]$

z-source

Softswitching DC-DC converter

- To minimize the intersection of their waveforms.
- A wide

voltage-gain range.

- Low-current ripple on the low-voltage side.

- Low voltage stresses across power switches.
- $\quad$ Fails to maintain better efficiency for a broad range of output voltages.

- Complex control and structure limit its application.
- $\quad$ Lower losses.

- Low EMI.

- Allows highfrequency operation.

- Additional clamping circuit

- $\quad$ is not required.
- Low fault tolerance capability.

- High current rating of gates.

- $\quad$ Large capacitor is required.
- Use the shoot-through (ST) state to boost the input voltage to higher. voltages to achieve high efficiency and high gain.
- $\quad$ Reduced cost and volume.

- High switching frequency.

- Low voltage stress on the devices.

- Good harmonic performance.
- Behave in a boost or buck operation only.

- $\quad$ The combined system of the DC-DC boost converter and the inverter has lower reliability.

- $\quad$ The main switching device of VSI and CSI are not interchangeable.
- Lower switching losses, especially when switching on the lowest valley point.

Quasi- - Minimizing converter resonant loss while optimizing converter

\section{multi targets.}

- $\quad$ Partial resonance with better EMI.

- High conversion ratio and efficiency.

- Low cost.
- $\quad$ The frequency increases as the load decreases.

- Complex integrated transformer.

- $\quad$ Expensive controller. 
Table 5. Cont.

\begin{tabular}{|c|c|c|c|c|}
\hline Ref. & $\begin{array}{c}\text { Type of } \\
\text { Converter }\end{array}$ & Target & Advantages & Disadvantages \\
\hline$[134,159]$ & $\begin{array}{l}\text { Multi-phase } \\
\text { DC-DC } \\
\text { converter }\end{array}$ & $\begin{array}{l}\text { Delivers continuous } \\
\text { input/output current } \\
\text { compared to } \\
\text { traditional converters. }\end{array}$ & $\begin{array}{l}\text { Higher efficiency from } \\
\text { lower transitional losses. } \\
\text { Lower output } \\
\text { ripple voltage. } \\
\text { Better } \\
\text { transient performance. } \\
\text { Lower } \\
\text { ripple-current-rating } \\
\text { requirements for the } \\
\text { input capacitor. }\end{array}$ & $\begin{array}{l}\text { Large number } \\
\text { of components. } \\
\text { - Switching converters } \\
\text { are prone to noise. } \\
\text { - Complex analysis } \\
\text { under steady-state and } \\
\text { transient conditions. } \\
\text { Difficult to achieve } \\
\text { proper synchronization. }\end{array}$ \\
\hline
\end{tabular}

In $[80,83,139]$, the cascaded multilevel converter is used to achieve high-quality output voltages and input currents. It has ease of operation at lower switching frequencies, less noise generation, and less harmonic distortion. However, this converter has a weakness of a higher number of power semiconductor switches. In [140,168], the buck-boost converter is employed to deliver a range of output voltages varying from much larger than the input voltage. It offers efficient solutions with smaller external components. Nevertheless, it needs a large filter size leading to electromagnetic interference (EMI) issues due to the discontinuous operation of the input current and charging current of the output capacitor. In [94], the neutral point clamp converter is used to achieve multilevel voltage waveform through a split DC-link, using multiple sources and storage through a single converter. Although this converter has improved waveform quality leading to reduced filtering requirements, it requires a greater number of diodes for increased levels. In [96], the matrix converter is utilized to achieve automatic conversion of power from AC to AC without DC-link capacitor. It provides a steady AC input source that can be converted directly into a variable voltage and variable frequency output. However, this converter is complex, less reliable, costly, and has limited capacity to produce the output voltage. In $[105,134,163]$, the modular multilevel converter is used for a wide range of medium and high-voltage applications. It has an adjustable power factor, flexible control operation, and smaller output harmonic content. Nonetheless, this converter requires a bulky and costly isolated transformer. In $[88,112,119]$, the interleaved boost converter is employed to achieve high efficiency and reliability. It offers numerous benefits, including faster transient response, high efficiency, low input current ripple, and reduced electromagnetic emission. Nonetheless, it possesses several disadvantages, such as high components count, high switching losses, and sensitivity to the duty cycle variation. In [113], the H-bridge converter allows DC motors to run forwards or backward. Although it has lower winding costs and proximity effect losses, it offers slightly more voltage ripple than the half-bridge. In $[86,90,98]$, the switched capacitor bidirectional converter is applied to attain a high step-up/step-down voltage gain. It has a wide voltage-gain range, low-current ripple on the low-voltage side, and low voltage stresses across power switches. However, it has some limitations, such as complex structure, control operation, and failure to maintain better efficiency for a broad range of output voltages. In $[117,127]$, the soft-switching DC-DC converter is used to minimize the intersection of their waveforms. It has lower losses, low EMI, and allows high-frequency operation. Nevertheless, it experiences a high current rating of gates and low fault tolerance capability. In [169], the z-source converter utilizes a shoot-through (ST) state to boost the input voltage to higher voltages to achieve high efficiency and high gain. This converter offers high switching frequency, good harmonic performance, low voltage stress, reduced cost, and volume. However, it has the drawbacks of lower reliability, vulnerability to EMI noises, and non-interchangeable voltage source inverter (VSI) and current source inverter (CSI) switching devices. In [77], a quasi-resonant 
converter is used to resemble a resonance action partially. This converter has a high conversion ratio, efficiency, and lower switching losses with better EMI. Nonetheless, it has shortcomings in terms of a complex integrated transformer and an expensive controller. In [134,159], a multi-phase DC-DC converter exhibits lower output ripple voltage, better transient performance, and lower ripple-current-rating requirements. Nevertheless, this converter suffers from higher components, synchronization, and sensitivity to duty cycle.

Table 6 presents the comparison of the technical features among various power electronics converters which are most frequently utilized in EV applications.

Table 6. Comparison of technical features of power electronics converters in EV applications.

\begin{tabular}{|c|c|c|c|c|c|c|}
\hline Type of Converter & $\begin{array}{c}\text { Current/Voltage } \\
\text { Ripple }\end{array}$ & $\begin{array}{l}\text { Switching } \\
\text { Frequency }\end{array}$ & $\begin{array}{l}\text { Complexity of } \\
\text { Control Circuit }\end{array}$ & $\begin{array}{l}\text { High Power } \\
\text { Conversion }\end{array}$ & $\begin{array}{c}\text { EMI } \\
\text { Suppression }\end{array}$ & Cost \\
\hline $\begin{array}{c}\text { Cascaded } \\
\text { multilevel converter }\end{array}$ & Complex & High & Moderate & Appropriate & Reduced & Low \\
\hline Buck-boost converter & Simple & High & Complex & Appropriate & Reduced & Medium \\
\hline $\begin{array}{l}\text { Neutral point } \\
\text { clamp converter }\end{array}$ & Moderate & Low & Moderate & Appropriate & Reduced & Low \\
\hline Matrix converter & Complex & High & Complex & Appropriate & Reduced & High \\
\hline $\begin{array}{l}\text { Modular } \\
\text { multilevel converter }\end{array}$ & Simple & High & Moderate & Appropriate & Needed & High \\
\hline $\begin{array}{c}\text { Interleaved } \\
\text { boost converter }\end{array}$ & Complex & Low & Complex & Appropriate & Reduced & Low \\
\hline H-bridge converter & Complex & High & Moderate & Appropriate & Needed & Low \\
\hline $\begin{array}{l}\text { Switched capacitor } \\
\text { bidirectional converter }\end{array}$ & Moderate & High & Moderate & Appropriate & Needed & Medium \\
\hline $\begin{array}{l}\text { Soft-switching } \\
\text { DC-DC converter }\end{array}$ & Complex & Low & Complex & Appropriate & Reduced & Medium \\
\hline Z-source converter & Simple & High & Complex & Appropriate & Needed & Medium \\
\hline $\begin{array}{c}\text { Quasi-resonant } \\
\text { converter }\end{array}$ & Simple & High & Moderate & Appropriate & Reduced & Low \\
\hline $\begin{array}{c}\text { Multi-phase } \\
\text { DC-DC converter }\end{array}$ & Complex & Low & Complex & Appropriate & Needed & High \\
\hline
\end{tabular}

3.5. Analysis of Energy Storage Connected Power Electronic Converter for EVs in Most Relevant 100 Papers

The selection of proper energy storage type or the combination of different storage devices plays a crucial role in EV applications. Figure 7 represents the different types of energy storage utilized in this most relevant selected 100 manuscripts. It is found that hybrid energy storage system (HESS) is the most widely used storage type in the selected database, with the figure of $66 \%$ followed by conventional battery storage type $(23 \%)$. Fuel cell storage and supercapacitor storage are the less utilized energy storage type in this selected database with the figure of $6 \%$ and $4 \%$, respectively. Flywheel energy storage is the lowest significant storage type with $1 \%$. According to selected papers, most of the researchers prefer hybrid energy storage in $\mathrm{EV}$ applications to ensure greater performance. 


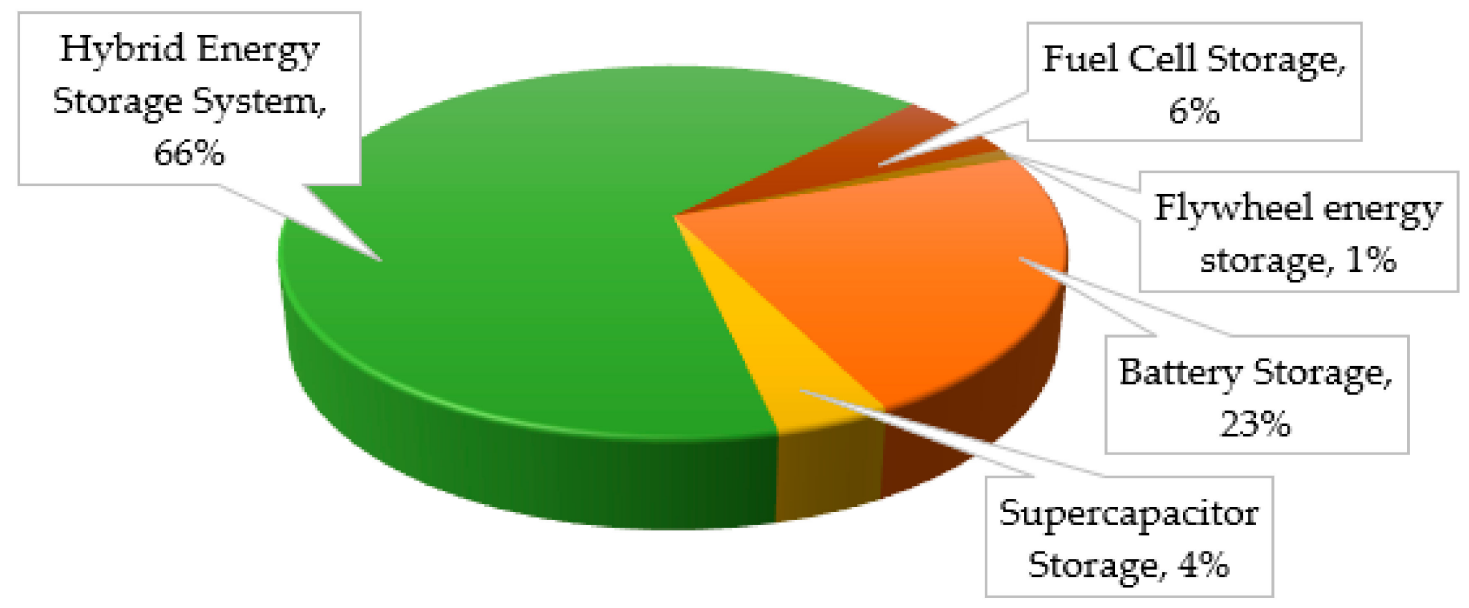

Battery Storage

⿴囗十 Hybrid Energy Storage System

Glywheel energy storage
Supercapacitor Storage

- Fuel Cell Storage

Figure 7. Classification of energy storage types from the selected database.

\subsection{Role of AI to Power Electronics Converter Technology Integrated Energy Storage Management}

Some recent works based on artificial neural network (ANN), fuzzy logic control, adaptive neuro-fuzzy inference system (ANFIS), and optimization techniques based on genetic algorithm (GA) and particle swarm optimization (PSO) are used in power electronics converter technology integrated energy storage management. Table 7 represents the various types of AI algorithms that are widely utilized in EVs along with their key findings and challenges. The following bullet points represent the benefit of the AI method in different EV applications:

- Yilmaz et al. [172] proposed an ANN-based half-bridge LLC resonant converter for EV technology. The presented technology delivered satisfactory outcomes with regard to long battery life and high efficiency resulting in better mileage for EVs. Mean square error (MSE) was considered to evaluate the performance of the ANN-based converter technology. The delivered results indicate that ANN-based technique achieved high efficiency with $96.2 \%$ with ripple voltage being $0.5 \mathrm{~V}$ and a total harmonic distortion (THD) of 5\%, respectively. While Teja et al. [173] presented A-based framework towards executing better power flow under two operational processes: the energy regeneration stage and the dual-source powering stage. The outcomes suggested high accuracy of the ANN model compared with the proportional-integral (PI) control technique with regards to fast response time and low harmonics.

- Ferreira et al. [174] introduced an integrated fuzzy logic control and power electronics converter circuits towards achieving high-performance efficiency in EV technology. Several energy storage systems such as battery storage, supercapacitor, and fuel cell were utilized where battery storage controls the input current while supercapacitor regulates DC-link voltage. The power flow control between the input sources and load was controlled with fuzzy interface. In other work, Adam et al. [175] presented a fuzzy logic control with converter topology towards achieving control for battery operation based on charging and discharging. The presented technique delivered adequate results regarding high battery capacity and long lifespan. Furthermore, the simulation outcome delivered high stability with output voltage parallel to high efficiency.

- The adaptive neuro-fuzzy inference system (ANFIS) is regarded as the integration of fuzzy systems and neural networks. The ANFIS depicts fast response and delivers results with high accuracy compared with traditional fuzzy based technique. Reddy and Sudhakar [176] developed a ANFIS controller for EV technology comprising of 
Fuel Cells, boost converter, three-phase inverter and motor respectively. The ANFIS technology was employed towards extracting maximum energy from the fuel cells. Additionally, the ANFIS operation demonstrated high superiority compared with conventional fuzzy logic control in terms of increased DC-link power and low average time to attain maximum power point.

- Some of the optimization techniques employed with power converters for EV technology relate to GA and PSO, respectively. For instance, Nguyen et al. [177] presented optimized GA-based dual active bridge (DAB) bidirectional converters for EV application. The main aim of the optimization framework was to maximize efficiency and minimize converter loss with low harmonics. The optimization framework varies several key parameters of transformer such as leakage inductance, peak flux density, voltage conversion ratio, and switching frequency. While Prithivi and Sathyapriya [178] developed improved the performance efficiency of fuzzy control technique with PSO. The achievement of the optimal value of switching angle was completed by eliminating current harmonics.

Table 7. AI methods in EV converters highlighting objectives, key findings, benefits, and shortcomings.

\begin{tabular}{|c|c|c|c|c|c|}
\hline $\begin{array}{c}\text { AI } \\
\text { Methods }\end{array}$ & Objectives & Key Findings & Benefits & Shortcomings & Refs. \\
\hline ANN & $\begin{array}{l}\text { Maximize power } \\
\text { factor, reduce } \\
\text { current harmonics. }\end{array}$ & $\begin{array}{c}\text { Achieved high } \\
\text { efficiency, low } \\
\text { harmonics, and THD. }\end{array}$ & $\begin{array}{l}\text { Demonstrates high } \\
\text { robustness } \\
\text { and accuracy. }\end{array}$ & $\begin{array}{l}\text { Requires high } \\
\text { computational } \\
\text { processor and } \\
\text { expensive } \\
\text { processor devices. }\end{array}$ & Yilmaz et al. [172] \\
\hline Fuzzy & $\begin{array}{c}\text { Maximize } \\
\text { efficiency and } \\
\text { develops } \\
\text { interfacing among } \\
\text { battery system, } \\
\text { generator, and } \\
\text { voltage } \\
\text { DC-link bus. }\end{array}$ & $\begin{array}{l}\text { Depicts appropriate } \\
\text { synchronization } \\
\text { between various } \\
\text { battery sources } \\
\text { and load. }\end{array}$ & $\begin{array}{l}\text { Presents high } \\
\text { robustness, } \\
\text { flexibility, and } \\
\text { fast response. }\end{array}$ & $\begin{array}{c}\text { Requires } \\
\text { appropriate } \\
\text { expertise towards } \\
\text { designing } \\
\text { the controller. }\end{array}$ & Ferreira et al. [174] \\
\hline ANFIS & $\begin{array}{l}\text { Maximize power } \\
\text { point tracking. }\end{array}$ & $\begin{array}{l}\text { Increase in DC link } \\
\text { power and reduction } \\
\text { in time required to } \\
\text { achieve maximum } \\
\text { power point. }\end{array}$ & $\begin{array}{l}\text { Highly effective } \\
\text { towards tracking. }\end{array}$ & $\begin{array}{c}\text { High } \\
\text { computational cost } \\
\text { with complex } \\
\text { structure and } \\
\text { gradient learning. }\end{array}$ & $\begin{array}{c}\text { Reddy and } \\
\text { Sudhakar [176] }\end{array}$ \\
\hline GA & $\begin{array}{l}\text { Maximization of } \\
\text { system efficiency. }\end{array}$ & $\begin{array}{l}\text { No requirement of } \\
\text { external inductors. }\end{array}$ & $\begin{array}{l}\quad \text { Requires less } \\
\text { information and fast } \\
\text { computational ability. }\end{array}$ & $\begin{array}{l}\text { Time consuming } \\
\text { and } \\
\text { unguided mutation. }\end{array}$ & Nguyen et al. [177] \\
\hline $\mathrm{PSO}$ & $\begin{array}{l}\text { Optimize energy } \\
\text { consumption. }\end{array}$ & $\begin{array}{l}\text { Reduction of effect of } \\
\text { control strategy. }\end{array}$ & $\begin{array}{l}\text { High robustness, } \\
\text { effectiveness, and } \\
\text { simple } \\
\text { implementation. }\end{array}$ & $\begin{array}{c}\text { Easy to fall into } \\
\text { local optimum and } \\
\text { low } \\
\text { convergence rate. }\end{array}$ & $\begin{array}{c}\text { Prithivi and } \\
\text { Sathyapriya [178] }\end{array}$ \\
\hline
\end{tabular}

\subsection{Analysis of Average Citation Per Year, Contributions, and Research Gaps}

Table 8 shows the top ten manuscripts with the average citation per year (ACY) in the recent five years. The manuscript by Cao et al. [81] has the highest ACY of 95.6 and is ranked 1st in total citations, followed by the second paper by Song et al., which has an ACY of 31.6. As the researchers' fields of interest change over time, the average citation per year (ACY) rank differs from the overall citation rank. With the ACY of 16, Kasimalla et al. [104] produce the last article in the table, whose citation rank is 24th. According to Table 4, the most frequent limitations are cost and relatively short lifetime for $\mathrm{LiFePO} 4$ battery, and the key weak point of FCs is their dynamic limitation. The fundamental disadvantage of the 
traditional technique is that non-linear properties are ignored, resulting in poor controller performance. The main limitation of the mentioned strategy is the use of a single DC bus and dynamic charging power limitation through G2V services; the inductive charging is the valid solution only for a short period, the mentioned control strategy cannot analyze the average positive and negative needs, and the hydrogen infrastructure, performance, reliability, and robustness are the future difficulties for FC systems. To overcome the issues mentioned above, further in-depth investigation is required. The future recommendations will be discussed in the following sections.

Cao et al. [81] applied a DC-DC converter connected with battery and supercapacitorbased hybrid energy storage system (HESS) in plug-in hybrid EVs and hybrid EVs. This scheme can utilize up to $75 \%$ of the ultracapacitor energy and generate a relatively constant battery load profile, leading to improved battery lifespan. However, the sizing of the DC-DC converter and the choice of the supercapacitor needs further exploration to reduce the system cost. Song et al. [82] employed a unidirectional DC-DC converter integrating a semi-active battery and ultracapacitor-based hybrid energy storage system in EV applications. This topology can minimize the battery capacity loss and system cost simultaneously. Although this approach is applicable for wide working temperatures, it has issues regarding cost and the short lifetime of LiFePO4 battery. Zheng et al. [83] used a hybrid cascaded multilevel converter for battery energy management and motor drives. This converter has lower harmonics and DV-DT, can efficiently utilize battery energy, and avoids the imbalance of SOC and voltage. Nahavandi et al. [84] designed a non-isolated multi-input multi-output DC-DC boost converter for EV applications using hybridized alternative energy sources. This converter has lower voltage harmonics leading to decrease torque ripple of the electric motor in EVs. El Fadil et al. [87] employed hybrid energy storage, including fuel cell and super-capacitor integrated with boost converter and boost-buck converter. A nonlinear control system is developed to track the supercapacitor current to its reference and regulate voltage regulation. Song et al. [89] introduced a fifth-order averaged model-based sliding-mode controller for a fully active hybrid energy storage system that employs two bi-directional DC-DC converters to decouple supercapacitor and battery pack from the DC bus. This converter can track DC bus voltage and the reference current of battery/SC under various load conditions. Akar et al. [90] proposed a new bidirectional non-isolated multi-input converter consisting of energy storage systems with various electrical properties in EVs. The proposed configuration has reduced components count, allowing a single switch connected with the converter's inductors. Experimental investigation indicates the prototype converter efficiency is above 93\%. Rubino et al. [91] emphasized the significance of charging infrastructure in large-scale diffusion of EV, highlighting inductive and conductive charging methods, innovative power electronics architectures, battery management, and integration of EV with distributed energy sources. The authors outlined several limitations such as slow charging, high dimension, oversizing, and complex hardware. Varajao et al. [96] proposed an improved control scheme for the high-frequency link matrix converter to achieve a controllable power factor for EV applications. This converter can generate high power quality grid currents as well as perform voltage and current regulation efficiently. Besides, this topology has lower weight and circuit volume and ensures a longer service life battery storage system. Kasimalla et al. [104] conducted a thorough investigation on the effectiveness of fuel cell EV (FCEV) based energy management systems connected with batteries and ultracapacitors. The various braking control schemes together with energy allocation, energy recovery regeneration, and experimental investigation are carried out to examine the hydrogen energy utilization. However, further attention is necessary on hydrogen infrastructure, performance, reliability, and robustness along with the FC system concerning size, cost weight, durability, and thermal management. 
Table 8. "Average citation per year (ACY)" of top 10 articles and their schemes, contributions, and research gaps.

\begin{tabular}{|c|c|c|c|c|c|c|c|c|c|}
\hline Rank & Ref. & Authors & $\mathrm{ACY}$ & $\begin{array}{l}\text { Rank } \\
\text { (Based on } \\
\text { Citation) }\end{array}$ & $\begin{array}{l}\text { Author } \\
\text { Keywords }\end{array}$ & Converter Type & Topology/Scheme & Contributions & $\begin{array}{l}\text { Research Gaps/Future } \\
\text { Directions }\end{array}$ \\
\hline 1 & [81] & Cao et al. & 95.6 & 1 & $\begin{array}{l}\text { Battery; Control; } \\
\text { DC-DC converters; } \\
\text { EV; ES; HEV; } \\
\text { PHEV; PE; } \\
\text { propulsion } \\
\text { systems; UC }\end{array}$ & DC-DC converter & $\begin{array}{l}\text { A novel battery and SC-based } \\
\text { hybrid energy storage system } \\
\text { (HESS) for electric drive cars, } \\
\text { such as plug-in hybrid electric } \\
\text { vehicles and hybrid } \\
\text { electric vehicles. }\end{array}$ & $\begin{array}{l}\text { Smaller size DC-DC } \\
\text { converter can transfer } \\
\text { energy utilizing up to } 75 \% \\
\text { of the } \\
\text { ultracapacitor energy. } \\
\text { A relatively constant load } \\
\text { profile is generated that } \\
\text { improves the battery life. }\end{array}$ & $\begin{array}{l}\text { - System efficiency in the } \\
\text { high voltage condition was } \\
\text { not analyzed. } \\
\text { The sizing of the DC-DC } \\
\text { converter and the selection } \\
\text { of the SC needs to figure } \\
\text { out to reduce the } \\
\text { system cost. }\end{array}$ \\
\hline 2 & {$[82]$} & Song et al. & 31.6 & 2 & $\begin{array}{l}\text { EV; HESS; } \\
\text { LiFePO4 battery } \\
\text { degradation; } \\
\text { multi-objective } \\
\text { optimization }\end{array}$ & Unidirectional DC-DC converter & $\begin{array}{l}\text { A semi-active battery and } \\
\text { ultracapacitor based hybrid } \\
\text { energy storage system for electric } \\
\text { vehicle applications. }\end{array}$ & $\begin{array}{l}\text { - The battery capacity loss } \\
\text { and system cost } \\
\text { are reduced. } \\
\text { The battery degradation is } \\
\text { decreased quickly with the } \\
\text { initial rise in } \\
\text { supercapacitor use. }\end{array}$ & $\begin{array}{l}\text { Cost and relatively short } \\
\text { lifetime are the main issue } \\
\text { of } \mathrm{LiFePO}_{4} \text { battery. } \\
\text { As the outcome of this } \\
\text { hybrid approach is } \\
\text { satisfactory for wide } \\
\text { working temperature, } \\
\text { hence, this model can be } \\
\text { utilized in future real } \\
\text { time applications. }\end{array}$ \\
\hline 3 & [83] & Zheng et al. & 27.6 & 3 & $\begin{array}{l}\text { BC; charging and } \\
\text { discharging; EV; } \\
\text { hybrid cascaded } \\
\text { multilevel } \\
\text { converter; } \\
\text { voltage balance }\end{array}$ & $\begin{array}{l}\text { Hybrid cascaded } \\
\text { multilevel converter }\end{array}$ & $\begin{array}{l}\text { A hybrid cascaded multilevel } \\
\text { converter is proposed, including } \\
\text { battery energy management and } \\
\text { motor drives. }\end{array}$ & $\begin{array}{l}\text { The type of converter has } \\
\text { lower harmonics and } \\
\text { dv/dt. Efficient utilization } \\
\text { of battery energy. } \\
\text { SOC and voltage } \\
\text { imbalance can be avoided. }\end{array}$ & $\begin{array}{l}\text { As the cascaded model has } \\
\text { better fault-tolerant ability } \\
\text { and no drawback on the } \\
\text { number of cascaded cells, it } \\
\text { can be utilized in the } \\
\text { real-time application. }\end{array}$ \\
\hline 4 & [84] & $\begin{array}{l}\text { Nahavandi } \\
\text { et al. }\end{array}$ & 26.8 & 4 & $\begin{array}{l}\text { DC-DC converters; } \\
\text { EV; hybrid power } \\
\text { system; MIMO; } \\
\text { small-signal } \\
\text { modeling; } \\
\text { state-space } \\
\text { averaging }\end{array}$ & $\begin{array}{l}\text { Non-isolated multi-input } \\
\text { multioutput DC-DC } \\
\text { boost converter }\end{array}$ & $\begin{array}{l}\text { A new non-isolated multi-input } \\
\text { multi-output DC-DC boost } \\
\text { converter for EV applications by } \\
\text { hybridizing alternative } \\
\text { energy sources. }\end{array}$ & $\begin{array}{l}\text { This converter can produce } \\
\text { several outputs with } \\
\text { different voltage levels. } \\
\text { Multilevel inverter reduces } \\
\text { the voltage harmonics } \\
\text { leading to decrease torque } \\
\text { ripple of electric motor } \\
\text { in EVs. }\end{array}$ & $\begin{array}{l}\text { As the proposed converter } \\
\text { was experimentally } \\
\text { verified, this strategy can } \\
\text { be utilized in practical } \\
\text { EV applications. }\end{array}$ \\
\hline
\end{tabular}


Table 8. Cont.

\begin{tabular}{|c|c|c|c|c|c|c|c|c|c|}
\hline Rank & Ref. & Authors & $\mathrm{ACY}$ & $\begin{array}{l}\text { Rank } \\
\text { (Based on } \\
\text { Citation) }\end{array}$ & $\begin{array}{l}\text { Author } \\
\text { Keywords }\end{array}$ & Converter Type & Topology/Scheme & Contributions & $\begin{array}{l}\text { Research Gaps/Future } \\
\text { Directions }\end{array}$ \\
\hline 5 & [87] & $\begin{array}{l}\text { El Fadil } \\
\text { et al. }\end{array}$ & 22.4 & 7 & $\begin{array}{l}\text { DC-DC power } \\
\text { converters; EV; FC; } \\
\text { nonlinear } \\
\text { control; SC }\end{array}$ & Bidirectional DC-DC converter & $\begin{array}{l}\text { A boost converter and a } \\
\text { boost-buck converter connected } \\
\text { with fuel cell and SC serving as } \\
\text { main power sources and } \\
\text { auxiliary power } \\
\text { source, respectively. }\end{array}$ & $\begin{array}{l}\text { A nonlinear control system } \\
\text { can track the SC } \\
\text { current accurately. } \\
\text { Can control DC } \\
\text { voltage regulation. }\end{array}$ & $\begin{array}{l}\text { - One of the key weak points } \\
\text { of FCs is their } \\
\text { dynamic limitation. } \\
\text { As the outcome of this } \\
\text { manuscript is batter, hence, } \\
\text { this strategy can be utilized } \\
\text { in practical application. }\end{array}$ \\
\hline 6 & [89] & Song et al. & 21.6 & 9 & $\begin{array}{l}\text { EV; HESS; } \\
\text { Lyapunov } \\
\text { function-based } \\
\text { controller; } \\
\text { sliding-mode } \\
\text { controller; SC }\end{array}$ & $\begin{array}{l}\text { Two bi-directional } \\
\text { DC-DC converters }\end{array}$ & $\begin{array}{l}\text { A sliding-mode controller } \\
\text { connected with two } \\
\text { bi-directional DC-DC converters } \\
\text { to decouple SC and battery pack } \\
\text { from the DC bus. }\end{array}$ & $\begin{array}{l}\text { - This converter can track } \\
\text { DC bus voltage and the } \\
\text { reference current of } \\
\text { battery/SC under various } \\
\text { load conditions. }\end{array}$ & $\begin{array}{l}\text { The fundamental } \\
\text { disadvantage of the } \\
\text { traditional technique is that } \\
\text { non-linear properties are } \\
\text { ignored, resulting in poor } \\
\text { controller performance. }\end{array}$ \\
\hline 7 & [90] & Akar et al. & 21.6 & 10 & $\begin{array}{l}\text { Batteries; } \\
\text { bidirectional; } \\
\text { HESS; } \\
\text { multi-input } \\
\text { converter; UC }\end{array}$ & $\begin{array}{l}\text { Bidirectional non-isolated } \\
\text { multi-input converter }\end{array}$ & $\begin{array}{l}\text { A new bidirectional non-isolated } \\
\text { multi-input converter consisting } \\
\text { of energy storage systems with } \\
\text { various electrical properties. }\end{array}$ & $\begin{array}{l}\text { The converter requires a } \\
\text { single switch connected } \\
\text { with inductors of the } \\
\text { converter leading to } \\
\text { reduced components count. } \\
\text { The prototype converter } \\
\text { efficiency is greater than } \\
\text { 93\% in both } \\
\text { operation modes. }\end{array}$ & $\begin{array}{l}\text { For future work, a } \\
\text { full-scale battery/UC } \\
\text { hybrid system can be built } \\
\text { using proposed converter. }\end{array}$ \\
\hline
\end{tabular}


Table 8. Cont.

\begin{tabular}{|c|c|c|c|c|c|c|c|c|c|}
\hline Rank & Ref. & Authors & $\mathrm{ACY}$ & $\begin{array}{l}\text { Rank } \\
\text { (Based on } \\
\text { Citation) }\end{array}$ & $\begin{array}{l}\text { Author } \\
\text { Keywords }\end{array}$ & Converter Type & Topology/Scheme & Contributions & $\begin{array}{l}\text { Research Gaps/Future } \\
\text { Directions }\end{array}$ \\
\hline 8 & [91] & Rubino et al. & 20.8 & 11 & $\begin{array}{l}\text { ESS; PEV charging } \\
\text { infrastructure; } \\
\text { PEV; PEC; SG }\end{array}$ & - & $\begin{array}{l}\text { A complete outlook for } \\
\text { innovative charging } \\
\text { infrastructures in } \\
\text { EV applications. }\end{array}$ & $\begin{array}{l}\text { Comparative analysis of } \\
\text { inductive and conductive } \\
\text { charging methods, } \\
\text { including scalability, } \\
\text { efficiency, and charging } \\
\text { time/power, are presented. } \\
\text { The off-board DC } \\
\text { fast-charging architectures } \\
\text { integrated and renewable } \\
\text { energy sources and energy } \\
\text { storage systems with the } \\
\text { main grid are discussed. }\end{array}$ & $\begin{array}{l}\text { - The main limitation of the } \\
\text { mentioned strategy is the } \\
\text { use of a single DC bus. } \\
\text { Dynamic charging power } \\
\text { limitation through } \\
\text { G2V services. } \\
\text { The inductive charging is } \\
\text { the valid solution only for } \\
\text { a short period. }\end{array}$ \\
\hline
\end{tabular}

AC-DC power

conversion; $\mathrm{BC}$

ES; matrix

$9 \quad[96] \begin{aligned} & \text { Varajao } \\ & \text { et al. }\end{aligned} \quad 16.75 \quad 16$

High-frequency link matrix

carbide; soft

switching; space

converter

vector pulse

width modulation
A novel modulation and control algorithms have been developed

for the high-frequency link matrix converter.
- This converter can generate grid currents with high power quality.

- $\quad$ The converter can perform voltage and current regulation efficiently.

- The total harmonic

distortion (THD) is 3.44\% and $2.58 \%$ in inverter mode and charge mode, respectively.
- $\quad$ As the outcome of this manuscript is excellent hence, this strategy can be utilized in real-world applications.
- $\quad$ The various braking control strategies to control strategies to improve hydrogen

This survey focuses on sever energy management schemes based on FCEV in conjunction with two secondary energy storage systems, such as batterie and SCs to create a

high-performance energy

storage system. battery; BDC; EM;

energy; UC (natteries explored.

- The experimental investigation, energy utilization, allocation plans, recovery of regeneration
- The hydrogen infrastructure, performance, reliability, and robustness are the future difficulties for FC systems.

- Need further studies of FC system with regard to size, cost weight, durability, thermal management. 


\subsection{Research Category in the Most Relevant 100 Papers}

Table 9 and Figure 8 demonstrate the research classification of the selected most relevant manuscripts. The relation among study type, year ranges, and citation ranges is also demonstrated. The category of modeling, simulation, and performance evaluation has the most papers $(57 \%)$, followed by problem formulation (13\%) and state-of-the-art and technical overview (9\%). With eight manuscripts each, review (systematic and nonsystematic) and survey, meta-analysis, and case study are in the fourth place together, where the citation range is $1-55$ and $0-133$, respectively. The observational overview has the lowest number of papers (5\%) with a citation range (15-81). The majority of papers from 2010 to 2021 are original research work (modeling, simulation, and performance evaluation) and problem formulation categories. In recent times, researchers are mostly focusing on the power electronics converter for the smooth power transition from energy storage to electric vehicle applications.

Table 9. Research types and citation range of the most relevant 100 publications based on the Scopus database.

\begin{tabular}{ccc}
\hline Types of Study & Year Range & Citation Range \\
\hline $\begin{array}{c}\text { Original research work (modeling, experimental } \\
\text { work, and performance evaluation) }\end{array}$ & $2010-2021$ & $0-809$ \\
\hline Problem formulation and simulation analysis & $2014-2021$ & $3-193$ \\
\hline State of art and technical overview & $2012-2020$ & $0-109$ \\
\hline Review (Systematic and Nonsystematic) & $2011-2021$ & $1-55$ \\
\hline Survey, Meta-analysis, and Case study & $2011-2021$ & $0-133$ \\
\hline Observational overview & $2013-2019$ & $15-81$ \\
\hline
\end{tabular}

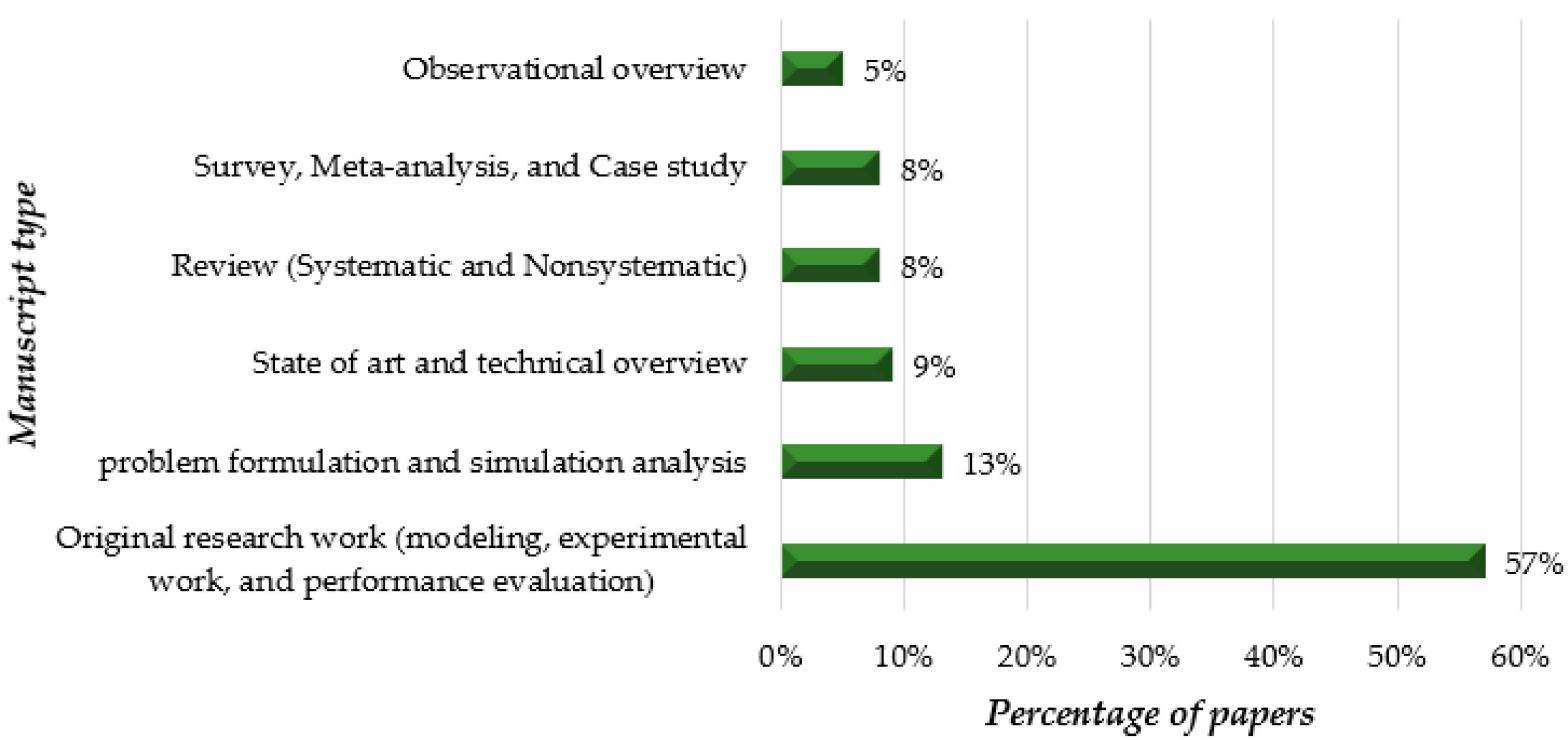

Figure 8. The percentage of manuscript types in the relevant top 100 cited papers.

\subsection{Analytical Evaluation of Journals and Publishers}

According to Figure 9, the selected most relevant 100 manuscripts were published under 17 different publishers. From Figure 9, future researchers will obtain a clear idea about the publisher, who frequently publishes the manuscripts related to energy storage management in EV applications. With 38 articles, the Institute of Electrical and Electronics Engineers published the highest number of publications from the selected 100 articles, where the maximum of them are original research work. Elsevier takes the second place 
with $19 \%$ of publications, MDPI AG with $10 \%$ and IET (5\%). The rest of the publishers published less than $5 \%$ of the selected manuscripts. The rest of the publishers are John Wiley \& Sons, Inc. (4\%), Korean Institute of Power Electronics (3\%), Inderscience Publishers (3\%), Korea Science (2\%), Taylor and Francis Inc. (2\%), Springer (2\%), Emerald Group Publishing Ltd. (1\%), Hindawi Publishing Corporation (1\%), SAGE Publications Ltd. (1\%), De Gruyter Open Ltd. (1\%), World Academy of Research in Science and Engineering (1\%), Research India Publications (1\%), and Tamkang University (1\%). Currently, scholars are focusing on the development of new energy storage management strategies and models in EV applications to reduce carbon emission, which has a lower environmental impact. Models based on EMSs for EV applications are investigated in Refs. [89,93,102,115,131,134,135].

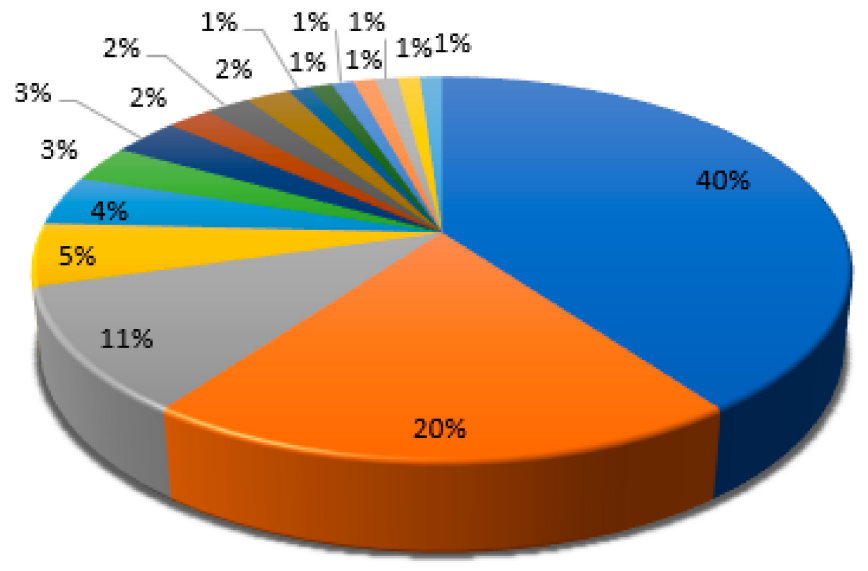

国IEEE $(38 \%)$

GMDP AG (10\%)

GJohn Wiley \& Sons, Inc. (4\%)

Inderscience Publishers (3\%)

国aylor and Francis Inc. (2\%)

Emerald Group Publishing Ltd. (1\%)

aSAGE Publications Ltd (1\%)

W World Academy of Research in Science and Engineering (1\%)

G Tamkang University (1\%)
国Elsevier (19\%)

IET $(5 \%)$

Gorean Institute of Power Electronics (3\%)

国Korea Science $(2 \%)$

G Springer $(2 \%)$

GHindawi Publishing Corporation (1\%)

ADe Gruyter Open Ltd (1\%)

Research India Publications ( $1 \%$ )

Figure 9. Distribution of 100 manuscripts over the different publishers.

The number of papers published in various journals, as well as their impact factor, is shown in Figure 10. The selected most relevant 100 manuscripts were published in 43 different journals. The top five journals with the highest number of publications have published $42 \%$ of the 100 most relevant manuscripts, with impact factors ranging from 1.069 to 14.982. The "IEEE Transactions on Power Electronics" journal published the highest number of papers (11), followed by "Energies" with ten manuscripts. "Applied Energy" and "IEEE Transactions on Industrial Electronics" both published eight and seven papers, respectively. Followed by "IEEE Transactions on Vehicular Technology" journal with the figure of six. "IET Power Electronics" and "Journal of Power Electronics" both published four articles each. "Energy Conversion and Management" and "International Journal of Energy Research" taken seventh place by publishing three articles each. The rest of the journals published less than three manuscripts from the selected database, and few of them are IEEE Access, IEEE Journal Of Emerging and Selected Topics In Power Electronics, IEEE 
Transactions On Applied Superconductivity, IEEE Transactions On Industry Applications, IEEE Transactions On Smart Grid, IEEE Transactions On Transportation Electrification, International Journal Of Power Electronics, Journal Of Electrical Engineering And Technology, Journal Of Power Sources, and Renewable and Sustainable Energy Reviews. Based on the journal citations report 2020, the selected 100 manuscripts published in the journal impact factor ranges from 1.069 to 14.982. "Renewable and Sustainable Energy Reviews" journal contain the highest IF of 14.982 , while the number of manuscripts is only $2 \%$. On the other hand, the "Journal of Electrical Engineering and Technology" journal with the same frequency of publication contains the lowest IF of 1.069.

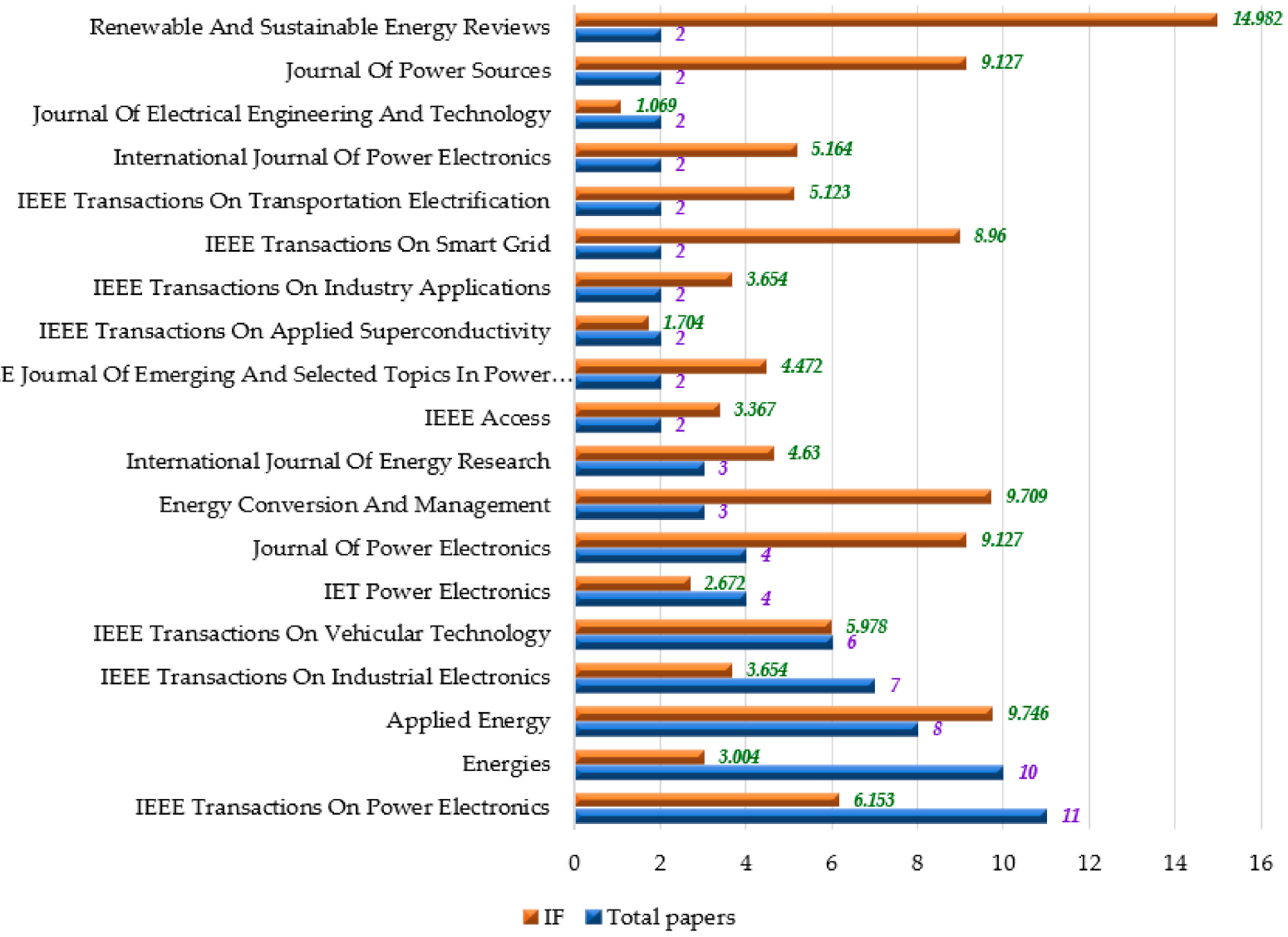

Figure 10. Distribution of manuscripts for Journal evaluation and Journal of impact factor.

\subsection{Document Authorship and Collaboration Analysis}

Table 10 depicts the research profiles of the prominent authors who have contributed three or more articles in the selected 100 manuscripts. From the most relevant 100 articles, ten authors produced more than three articles. Amjadi, Zahra of the Florida Polytechnic University, United States, is the leading author with six publications as a first author. Williamson, Sheldon S., and $\mathrm{Xu}$, Jun published five manuscripts each from the Ontario Tech University, Canada and State Key Laboratory for Manufacturing Systems Engineering, China, respectively. This is followed by Binggang Cao, Clemente Capasso, Ottorino Veneri, and Bin Wang, who each published four articles from the Xi'an Jiaotong University, China and Istituto Motori, Italy, respectively. The rest of the authors published three articles each. In terms of citations, Josep M. Guerrero from the Aalborg University, Denmark takes the first place with 55862 citations and an h-index of 104. This is followed by Williamson, Sheldon S., and Cao, Binggang, with citations of 5635 and 3374, respectively. Among the topmost ten authors, four of them are from China. Figure 11 presents the co-authorship and collaboration analysis using the VOSviewer software tool. It is observed that the highest number of authors participated from China, followed by India. 
Table 10. Topmost 10 prominent authors profiles based on most relevant 100 manuscripts.

\begin{tabular}{|c|c|c|c|c|c|c|c|}
\hline Rank & Author Name & Present Affiliation & $\begin{array}{l}\text { Country } \\
\text { Name }\end{array}$ & $\begin{array}{l}\text { Number of } \\
\text { Articles }\end{array}$ & $\begin{array}{l}\text { Total Number } \\
\text { of Citations }\end{array}$ & h-Index & Authors Position \\
\hline 1 & Amjadi, Zahra & $\begin{array}{c}\text { Florida } \\
\text { Polytechnic University }\end{array}$ & United States & 6 & 457 & 7 & First author $=6$ \\
\hline 2 & $\begin{array}{l}\text { Williamson, } \\
\text { Sheldon S. }\end{array}$ & $\begin{array}{c}\text { Ontario } \\
\text { Tech University }\end{array}$ & Canada & 5 & 5635 & 36 & Senior author $=5$ \\
\hline 3 & $\mathrm{Xu}$, Jun & $\begin{array}{l}\text { State Key Laboratory } \\
\text { for Manufacturing } \\
\text { Systems Engineering }\end{array}$ & China & 5 & 1945 & 21 & Co-author $=5$ \\
\hline 4 & Cao, Binggang & $\begin{array}{c}\text { Xi'an } \\
\text { Jiaotong University }\end{array}$ & China & 4 & 3374 & 28 & $\begin{array}{c}\text { Senior author }=2 \\
\text { Co-author }=2\end{array}$ \\
\hline 5 & Capasso, Clemente & Istituto Motori & Italy & 4 & 619 & 12 & $\begin{array}{c}\text { First author }=2 \\
\text { Co-author }=2\end{array}$ \\
\hline 6 & Veneri, Ottorino & Istituto Motori & Italy & 4 & 1233 & 21 & $\begin{array}{c}\text { First author }=1 \\
\text { Senior author }=3\end{array}$ \\
\hline 7 & Wang, Bin & $\begin{array}{l}\text { State Key Laboratory } \\
\text { for Manufacturing } \\
\text { linebreak } \\
\text { Systems Engineering }\end{array}$ & China & 4 & 571 & 11 & First author $=4$ \\
\hline 8 & Guerrero, Josep M. & Aalborg University & Denmark & 3 & 55862 & 104 & $\begin{array}{c}\text { Co-author }=1 \\
\text { Senior author }=2\end{array}$ \\
\hline 9 & Song, Ziyou & $\begin{array}{l}\text { University } \\
\text { of Michigan }\end{array}$ & United States & 3 & 1970 & 22 & $\begin{array}{c}\text { First author }=2 \\
\text { Co-author }=1\end{array}$ \\
\hline 10 & Yin, Chengliang & $\begin{array}{c}\text { Shanghai } \\
\text { Jiao Tong University }\end{array}$ & China & 3 & 1821 & 20 & $\begin{array}{c}\text { Co-author }=2 \\
\text { Senior author }=1\end{array}$ \\
\hline
\end{tabular}

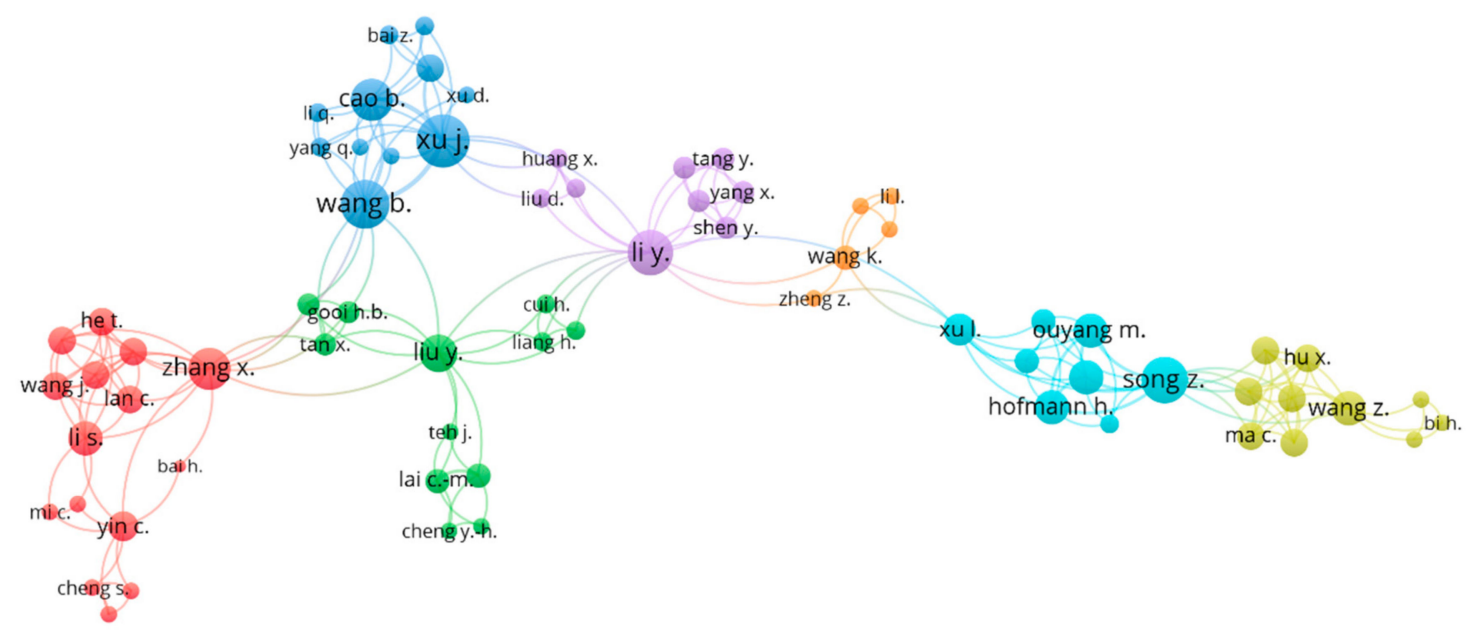

Figure 11. VOSviewer illustration for co-authorship analysis using Scopus database.

Research interests vary from author to author. Zahra Amjadi from Florida Polytechnic University, Lakeland, United States, primarily focuses on power electronics, electric vehicles, hybrid electric vehicles, transportation electrification, and electric energy storage systems. She recently published couple of research papers on energy storage management in EV applications [98,103,116,122,165,166]. Her most recent two journals are "Digital Control of a Bidirectional DC-DC Switched-Capacitor Converter for Hybrid Electric Vehicle Energy Storage System Applications" [116] and "Prototype Design and Controller Implementation 
for a Battery-Ultracapacitor Hybrid Electric Vehicle Energy Storage System" [122]. Sheldon S. Williamson from Ontario Tech University, Oshawa, Canada, is currently working on the following fields: batteries and charging, electric machines, electric vehicles, energy harvesting, energy storage systems, motor drives, power electronics, renewable energy, and transportation electrification $[98,103,116,122,165,166]$. His most recent two articles are "Modeling of Average Current in Non-Ideal Buck and Synchronous Buck Converters for Low Power Application" [122] and "Technological Perspective of Cyber-Secure Smart Inverters Used in the Power Distribution System: State of the Art Review" [179]. Jun Xu from State Key Laboratory for Manufacturing Systems Engineering, Xi'an, China, takes third place in selected most relevant 100 manuscripts. His primary research interests are inductive power transmission, charging (batteries), electric vehicles, high voltage direct current systems, converters, static synchronous compensators, space vector modulation, and inverter $[92,95,115,180,181]$. "A Relative State of Health Estimation Method Based on Wavelet Analysis for Lithium-Ion Battery Cells" [182] and "A Hybrid Self-Heating Method for Batteries Used at Low Temperature" [183] are the articles recently generated by Jun $\mathrm{Xu}$.

\subsection{Country Analysis in the Most Relevant 100 Papers}

Figures 12 and 13 present the graphical representation of the top ten countries and cooccurrence countries using VOSviewer that dominate the power converter integrated ESM in EV applications, respectively. China has taken first place by publishing 33 manuscripts followed by India, with 17 manuscripts. The United States is in the third position with 14 articles. With one publication, South Korea is placed in the last position based on our selected database. Figure 13 represents the co-occurrence network among all the countries, who published the selected 100 articles. Moreover, Figure 13 illustrates that China has the highest number of links with other countries, followed by Malaysia and the United States. Portugal and Germany have the lowest number of collaboration networks from the selected database. "National Natural Science Foundation of China" is the sponsor of the highest number of manuscripts from the selected most relevant 100 articles followed by "European Regional Development Fund" and "European Regional Development Fund" that is placed at second position with three manuscripts each.

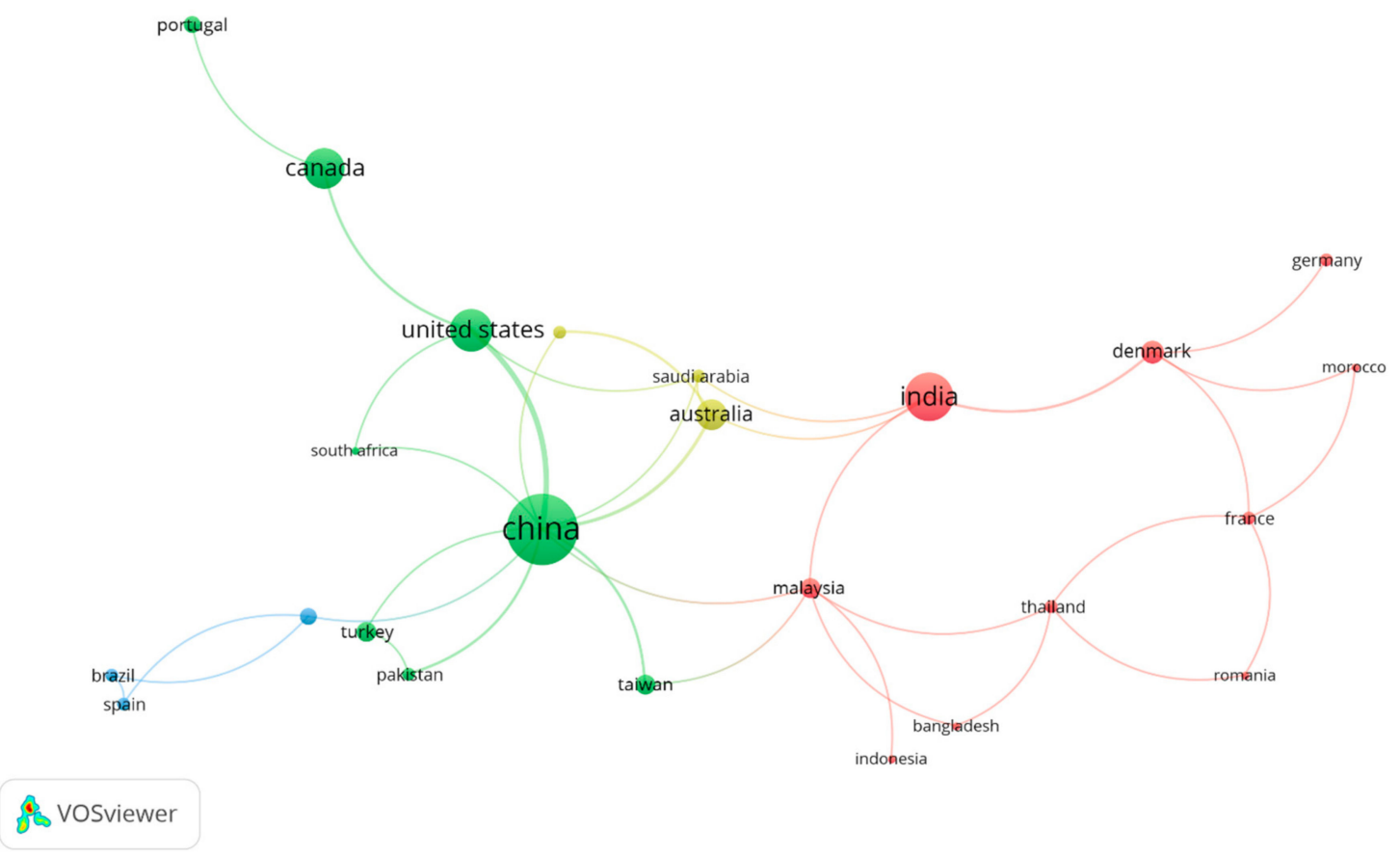

Figure 12. Graphical representation of top ten countries that dominate the EMSs for EV application. 


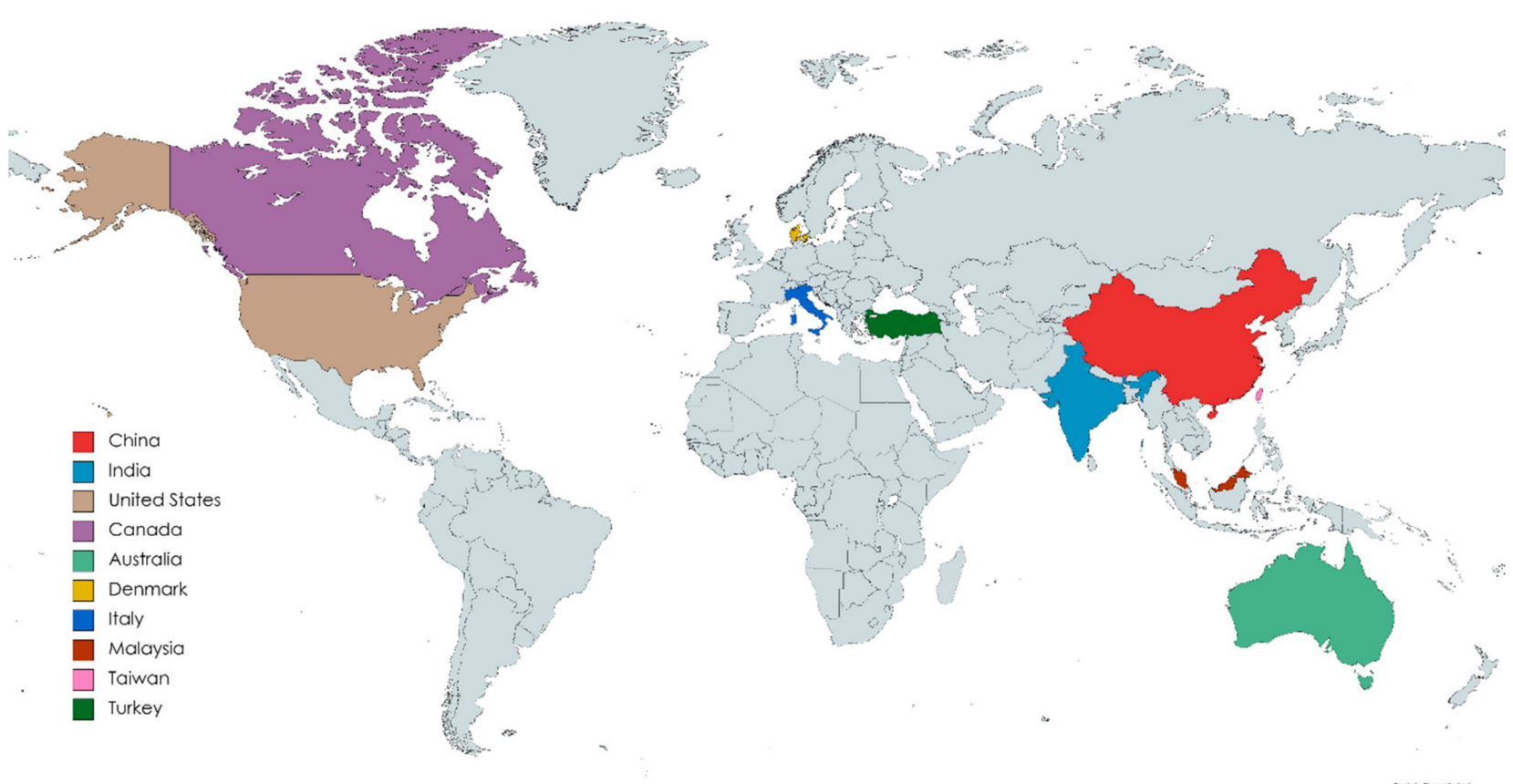

Figure 13. Co-occurrence country analysis by VOSviewer from the Scopus database.

\section{Issues and Challenges of EV Converters}

Even though various efforts have been initiated to develop state-of-the-art converter technologies with regards to design, operation control, and optimization, numerous key issues should be addressed towards enhancing the converter performance accuracy. Table 11 represents the key challenges with different EV application converters along with their impacts and possible suggestions/solutions. To address this, the key issues and challenges have been proposed as:

Table 11. Key challenges and possible solutions of EV converter technology.

\begin{tabular}{|c|c|c|c|c|}
\hline No. & Issues and Challenges & Causes & Impacts & Remedies/Suggestions \\
\hline 1 & $\begin{array}{l}\text { EV Converter Topology } \\
\text { and Design Criteria }\end{array}$ & $\begin{array}{l}\text {-Lack of an appropriate } \\
\text { design and configuration. } \\
\text {-Application of filter } \\
\text { circuits increases the } \\
\text { converter cost. }\end{array}$ & $\begin{array}{l}\text { Presence of unwanted } \\
\text { harmonics, voltage stress, } \\
\text { improper synchronization, } \\
\text { and switching loss. }\end{array}$ & $\begin{array}{c}\text { GA / PSO } \\
\text { optimization-based } \\
\text { bidirectional converters for } \\
\text { EVs can be employed to } \\
\text { enhance efficiency with low } \\
\text { output current ripple and } \\
\text { converter loss. }\end{array}$ \\
\hline 2 & $\begin{array}{l}\text { Traditional Controller } \\
\text { Implementation Issues }\end{array}$ & $\begin{array}{l}\text { Slow response rate to } \\
\text { sudden disturbances and } \\
\text { highly sensitive to } \\
\text { controller gains. }\end{array}$ & $\begin{array}{l}\text { Delivers unsatisfactory } \\
\text { outcomes for highly } \\
\text { non-linear and } \\
\text { time-varying systems. }\end{array}$ & $\begin{array}{c}\text { Various AI controller } \\
\text { schemes can be employed } \\
\text { towards controlling DC-link } \\
\text { voltage and load current and } \\
\text { achieving better } \\
\text { power management. }\end{array}$ \\
\hline 3 & $\begin{array}{l}\text { Intelligent EV Controller } \\
\text { Complexities Issues }\end{array}$ & $\begin{array}{l}\text { Requirement of large } \\
\text { volume of data and } \\
\text { human expertise. }\end{array}$ & $\begin{array}{l}\text { Presents inadequate stability } \\
\text { control and } \\
\text { dynamic response. }\end{array}$ & $\begin{array}{l}\text {-Appropriate selection of } \\
\text { suitable hyperparameters. } \\
\text {-Utilization of high } \\
\text { computational processors. }\end{array}$ \\
\hline
\end{tabular}


Table 11. Cont.

\begin{tabular}{|c|c|c|c|c|}
\hline No. & Issues and Challenges & Causes & Impacts & Remedies/Suggestions \\
\hline 4 & $\begin{array}{l}\text { Optimization } \\
\text { Framework Issues }\end{array}$ & $\begin{array}{c}\text { Suitable parameters } \\
\text { selection and proper } \\
\text { optimization technique. }\end{array}$ & $\begin{array}{l}\text { Increase system complexity } \\
\text { and computational cost. }\end{array}$ & $\begin{array}{l}\text { Application of advanced } \\
\text { optimization framework } \\
\text { with low complexity and } \\
\text { high effectiveness } \\
\text { is required. }\end{array}$ \\
\hline 5 & $\begin{array}{c}\text { Development of } \\
\text { Multi-Objective Function }\end{array}$ & $\begin{array}{l}\text { Quite tedious and } \\
\text { challenging to formulate } \\
\text { multi-objective function }\end{array}$ & $\begin{array}{l}\text { Less possibility to achieve } \\
\text { optimal results with ripple } \\
\text { current and number of } \\
\text { components simultaneously. }\end{array}$ & $\begin{array}{l}\text { More realistic parameters, } \\
\text { including design, cost, } \\
\text { energy loss, and related } \\
\text { constraints need to } \\
\text { be considered. }\end{array}$ \\
\hline 6 & $\begin{array}{c}\text { Application of } \\
\text { Metaheuristic Techniques }\end{array}$ & $\begin{array}{l}\text { Complex mathematical } \\
\text { computations with several } \\
\text { hyperparameter adjustments. }\end{array}$ & $\begin{array}{l}\text { Acquisition of unsatisfactory } \\
\text { outcomes with regard to } \\
\text { convertor loss and } \\
\text { components } \\
\text { cost respectively. }\end{array}$ & $\begin{array}{l}\text { The computational } \\
\text { complexity can be reduced } \\
\text { with suitable selection } \\
\text { of hyperparameters. }\end{array}$ \\
\hline 7 & $\begin{array}{l}\text { Execution of Intelligent } \\
\text { Controller Schemes }\end{array}$ & $\begin{array}{l}\text { Requirement of suitable } \\
\text { input features, network } \\
\text { configuration and } \\
\text { hyperparameter tuning. }\end{array}$ & $\begin{array}{l}\text { Loss of time and } \\
\text { human energy. }\end{array}$ & $\begin{array}{l}\text { Further studies are required } \\
\text { to reduce the data training } \\
\text { operation and } \\
\text { hyperparameter settings of } \\
\text { intelligent controllers. }\end{array}$ \\
\hline 8 & $\begin{array}{l}\text { Battery Storage } \\
\text { Integration Issues }\end{array}$ & $\begin{array}{l}\text { Improper converter } \\
\text { operation, complex control, } \\
\text { and modulation. }\end{array}$ & $\begin{array}{l}\text { Heat dissipation, battery } \\
\text { over-charging/ } \\
\text { over-discharging issues. }\end{array}$ & $\begin{array}{l}\text { Appropriate selection of } \\
\text { components and } \\
\text { converter levels. }\end{array}$ \\
\hline
\end{tabular}

\subsection{EV Converter Topology and Design Criteria}

In Refs. $[73,81,95,96,107,117,139,160]$, numerous authors have demonstrated various converter topologies and their operational features for EV application. The performance of EVs can be enhanced by employing an appropriate converter design. Undesirable outcomes can occur during the inappropriate selection of converter topology for the desired requirement. The inclusion of a large number of switching components may result in more weight to the converter. Furthermore, the application of filter circuits could increase the converter cost. Additional to this, various other converter operational issues such as the presence of unwanted harmonics, voltage stress, improper synchronization, and switching loss may deteriorate the performance and accuracy. Henceforth, an effective converter topology for EV application needs further investigation.

\subsection{Traditional Controller Implementation Issues}

In Ref. [131], the author explored the characteristics features of the conventional controller in EV applications. The traditional controller such as PI controller and PID controller has been utilized in EV applications. The PI controller depicts simple topology and execution, delivers satisfactory outcomes but shows some disadvantages such as slow response rate to sudden disturbances and highly sensitive to controller gains. While PID controller demonstrates better feasibility and easy implementation, it delivers unsatisfactory outcomes for highly non-linear and time-varying systems. Furthermore, the transient response of the PID controller with time-delayed circuits is sluggish.

\subsection{Intelligent EV Controller Complexities Issues}

In Refs. $[75,89,126,148,160]$, the authors discussed various aspects of the intelligent EV controller design and operational features. Various controllers in EV application such as fuzzy controller, ANN controller, SM controller, etc. With regards to a fuzzy controller and ANN controller, satisfactory results have been achieved with efficient and better control of converters. The fuzzy controller is effective, robust, flexible, and delivers a better transient 
response, and is therefore utilized in the EV control application. However, fuzzy controllers have various weaknesses, such as the requirement of a large volume of data, regular upgradation of rules, and human expertise. On the other hand, the ANN controller delivers effective results, requires no mathematical model, and operates satisfactorily under various loading conditions. Nonetheless, ANN requires a large volume of training datasets to train the ANN model and high computational processors. The sliding mode (SM) control technique delivers stability, reliability, and robustness but suffers from fluctuations in switching frequency and requires appropriate suitable parameter selection.

\subsection{Optimization Framework Issues}

In Refs. $[73,82,160]$, various authors discussed the application of optimization algorithms for optimal outcomes in terms of performance enhancement and design optimization. The applicability of various optimization frameworks in EV applications significantly contributes to achieving optimized design, performance, and accuracy. Nonetheless, the inclusion of the optimization techniques has resulted in some negative impacts in terms of suitable parameter selection. For instance, genetic algorithm (GA) is easy to implement and performs satisfactorily with parallel searching ability, but requires large computational time due to slow convergence, whereas particle swarm optimization (PSO) presents easy implementation, requires few hyperparameter adjustments, and shows fast convergence to achieve optimal results. Nevertheless, the premature convergence and assigning appropriate values for the design parameters in PSO delivers undesirable outcomes. Henceforth, satisfactory results with optimization techniques in EV application can be achieved with proper metaheuristic optimization techniques.

\subsection{Development of Multi-Objective Function}

The development of MOF in EV application is a critical step for designing the optimization technique due to the formulation of the objective function with several variables and constraints. In a converter circuit, the optimal performance can be achieved with the optimization of various parameters such as the number of components, switching losses, current, and voltage. To accomplish the task, the construction of MOF is required. However, the acquisition of two optimal solutions simultaneously is quite tricky and could result in delivering unsatisfactory outcomes. Henceforth, further studies need to be conducted for exploring the construction of MOF and its related constraints.

\subsection{Application of Metaheuristic Techniques}

The implementation of metaheuristic techniques to control EV converters may deliver undesirable outcomes due to complex mathematical computations with several hyperparameter adjustments. The training time in metaheuristic optimization techniques is long and requires complex mathematical expressions. Furthermore, the performance accuracy among various optimization schemes by utilizing a single convergence curve is difficult. Additionally, the hybridization of optimization may lead to unsatisfactory outcomes in a case when insufficient data is provided along with other procedures such as data preprocessing, suitable selection of hyperparameters not performed appropriately. Therefore, further investigation is required to address the complexity of optimization techniques.

\subsection{Execution of Intelligent Controller Schemes}

The performance accuracy of intelligent controllers depends on various factors such as appropriate selection of input data, model configuration, and selection of suitable hyperparameters. The performance accuracy of the converter is increased by accurately optimizing the hyperparameters. In some work, the membership function and hyperparameter adjustment were not performed with fuzzy and ANN controllers $[98,119]$. Additionally, the implementation of the hit and trial method for selecting the hyperparameters for intelligent controllers requires significant time and human energy. Henceforth, the issues related to intelligent controller schemes' design and execution need further exploration. 


\subsection{Battery Storage Integration Issues}

The energy storage system comprises several battery technologies such as the lithiumion battery, supercapacitor, and fuel cell, respectively [184]. Even though the lithium-ion battery has been extensively utilized in several applications, nevertheless, the battery displays low robustness and suffers from aging [185]. While supercapacitor technology presents benefits such as fast charging time and long life cycle, however, the supercapacitor is not suitable for long-term usage [186]. On the other hand, fuel cell technology shows zero carbon emission but demonstrates complexity to store at ambient room temperature and pressure [187]. Commonly, energy storage technologies suffer from issues such as aging, charging/discharging, uncertainties, limited availability, and high costs [188]. Furthermore, the integration of energy storage system with power convertor circuitry indicates some critical issues. For instance, when the energy storage system is integrated with two-level full-bridge converters topology, it may distort output waveform due to the operation of converter topology as a buck converter. Additionally, when energy storage technology is integrated with a three-level neutral-point clamped (NPC) converter, it may suffer from complex control. However, the battery integration issue with power converters can be suitably resolved with appropriate selection of components and converter levels, respectively [189].

\section{Future Research Opportunities for EV Converters}

The manuscript provides several effective and fruitful suggestions for improving PEC in EV applications which are mentioned below.

- Usually, the converter circuits present a high magnitude of switching and power loss in passive components. At present, various wide bandgap (WBG) material compositions such as silicon carbide $(\mathrm{SiC})$ and gallium nitride $(\mathrm{GaN})$ are utilized in EV converter development due to their capability for handling high voltage and current and delivering high power density while dissipating low heat. Nonetheless, the material is non-reliable and expensive. Thus, the implementation of these sophisticated materials for EV applications should be given more priority in future research.

- Apart from the usage of wide bandgap (WBG) materials such as $\mathrm{SiC}$ and $\mathrm{GaN}, \mathrm{a}$ significant amount of emphasis is currently given on the ultra-wide bandgap (UWBG) materials like $\mathrm{Al}(\mathrm{Ga}) \mathrm{N}$ and $\mathrm{Ga}_{2} \mathrm{O}_{3}$ since they have even higher power density and can be applied in high power applications. Although fabrication of UWBG materials is still in its early stage, they have the potential to be applied as switches of DC-DC converters that can bring many advantageous aspects for EV applications. Therefore, further research study should be carried out towards selecting appropriate material composition for developing suitable converter for EV application with better reliability, low cost, and high switching frequency.

- The schemes of the various converter topologies face various issues related to high harmonics in output current, low current and voltage stress, and low impedance, respectively. Henceforth, further investigation is needed for improving the electrical design features to achieve high frequency and low converter loss. Furthermore, studies related to mechanical design optimization should be conducted to obtain better reliability and accuracy.

- The importance of external design schemes is as important as the internal design features of converters. Improving the internal electric design features are not alone enough to bring expected results and improvement. Besides, changing the internal electric design is often brings unwanted complexity and is extremely time-consuming and expensive. In this regard, significant numbers of studies are conducted to develop various passive and active power filters that can improve the performance of converters externally. The major advantage of these power filters is that they can be easily built with cheap power electronic components and can drastically reduce high harmonics, disturbances, and noises in the output signals of the converters. Thus, 
similar importance should be offered in researching the developments of power filters for converters in EV applications.

- The employment of multilevel multi-phase bidirectional converters in EV technology has seen a significant rise due to their various aspects such as low current stress, easy control mechanism, and high-performance efficiency. However, further research work should be performed to investigate the requirements of additional components and complex analysis under steady-state and transient conditions. Furthermore, the converters present high sensitivity in the duty cycle towards variable loading conditions. Therefore, it is suggested to concentrate on developing an integrated design framework to achieve better scalability and fidelity, respectively.

- The application of an intelligent control mechanism is utilized in controlling DC link voltage with better power management, fast-tracking capability, and high-performance efficiency. Nevertheless, they suffer from various shortcomings, such as a complex training process, which requires large training time and an appropriate selection of hyperparameters. Henceforth, future study is needed to address various issues with intelligent control schemes.

- $\quad$ Even though various metaheuristic optimization techniques are benefitted by minimizing the components, converter loss, and cost, their implementation in EV application is limited. To date, two optimization techniques, i.e., GA and PSO, have been utilized for optimizing design and cost. Therefore, it is recommended to implement other advanced and hybridized optimization techniques in EV applications.

- Parameter specification of the electric power components applied in the converters is one of the most effective ways of ensuring optimized performance. Selecting and deciding these parameters manually is both difficult and time-consuming. Besides, they often do not bring expected results in terms of performance. For this purpose, advanced optimization techniques and machine learning techniques can be applied to select the parameters of the power electronic components in converters and accurately predict the performances of the converters. Hence, future research needs to be more focused on developing such techniques for converters in EV applications.

- Machine learning techniques are currently under vast study for predicting and analyzing various types of faults in power converters, such as short circuit and open circuit faults. Accurate prediction of these faults is highly important since it can protect the converters from physical damage and prevent other hazardous incidences. As a result, the development of machine learning techniques is of high importance in terms of the implementation of power converters in EV applications.

The aforementioned suggestions could pave the way to develop and execute stateof-the-art converters in EV applications. Furthermore, this review work will encourage the researchers and automotive industries to conduct future research work on developing intelligent converters in the automotive field with better design configuration and operational capabilities. Overall, this review helps to achieve a pathway for future sustainable EV expansions.

\section{Conclusions}

The integration of energy storage management and power electronic converter improves the overall performance of EVs technology regarding EVs internal structure development, motor speed and torque regulation, voltage compensation, voltage boost, and power flow control. The focal point of this study is to come up with an academic research culture overview and highlight the characteristics and development of power electronic converter integrated energy storage management technology in EV applications through top-notch research articles. The key findings of this review are summarized below.

- This work explores the most related 100 research works published in the last ten years on ESSs integrated converter technology in EV applications from the database of Scopus by assessing the current trends, operation, applications, and problems. 
- Several exclusive sections are created based on the most popular keywords, top highly cited research works, and top contributed authors. Additional insights are provided on the research articles by allocating them in terms of the year, citation, publishers, journal impact factor, and nations.

- It was investigated that the highest cited paper belongs to Cao et al. published in IEEE Transactions on Power Electronics 2012 from the USA with 809 citations.

- The most prominent author was Zahra Amjadi, with a total of six publications as the first author and constituting 457 citations with $7 \mathrm{~h}$-index. While the second position was secured by Sheldon S. Williamson with 5635 citations in five publications

- China secured the first position in terms of country analysis with 33 publications followed by India with 17 and USA with 14 respectively.

- An in-depth investigation is carried out to examine the emerging power electronic converter topologies, targets, advantages, disadvantages, schemes, contributions, and research gaps. Several key challenges and feasible solutions are discussed, focusing on the converter design, controller operation, intelligent controllers design, and metaheuristic techniques.

- Comprehensive and insightful remarks for future research opportunities are delivered towards the advancement of EVs.

Finally, it is desired that the shared research works concepts, deliberations, and invaluable statistics on converter and energy storage management technology in EV applications will facilitate magnifying EV implementation and contribute to the automobile industry on the road to achieving sustainable development goals (SDGs) and decarbonization.

Author Contributions: Conceptualization, M.S.H.L.; methodology, M.S.H.L. and M.S.M.; formal analysis, M.S.H.L. and M.S.M.; investigation, M.S.H.L. and M.S.M.; resources, M.S.H.L.; data curation, M.S.M.; writing—original draft preparation, M.S.H.L., M.S.M., S.A., S.T.M. and K.H.; writingreview and editing, M.S.H.L., R.M.E., A.A.M., M.A.A.M.Z. and A.H.; supervision, M.S.H.L.; project administration, M.S.H.L.; funding acquisition, M.S.H.L. All authors have read and agreed to the published version of the manuscript.

Funding: This research received no external funding.

Conflicts of Interest: Declare conflicts of interest.

$\begin{array}{ll}\text { Abbreviations } & \\ \text { ACY } & \text { Average citation per year } \\ \text { AI } & \text { Artificial Intelligence } \\ \text { ANN } & \text { Artificial neural network } \\ \text { ANFIS } & \text { Adaptive neuro-fuzzy inference system } \\ \text { BDC } & \text { Bidirectional Converter } \\ \text { BMS } & \text { Battery Management Systems } \\ \text { BP } & \text { Battery Pack } \\ \text { BS } & \text { Battery Storage } \\ \text { CMV } & \text { Common-mode voltage } \\ \text { CS } & \text { Capacitor Storage } \\ \text { CSI } & \text { Current source inverter } \\ \text { EB } & \text { Electric Batteries } \\ \text { EE } & \text { Energy Efficiency } \\ \text { EES } & \text { Electric Energy Storage } \\ \text { EI } & \text { Electric Inverters } \\ \text { ELF } & \text { Electric Load Flow } \\ \text { EM } & \text { Energy Management } \\ \text { EMC } & \text { Electric Machine Control } \\ \text { EMI } & \text { Electromagnetic interference } \\ \text { EMS } & \text { Energy Management Systems } \\ \text { EPSC } & \text { Electric Power System Control } \\ & \end{array}$




\begin{tabular}{|c|c|}
\hline EPTN & Electric Power Transmission Networks \\
\hline ES & Energy Storage \\
\hline ESM & Energy storage management \\
\hline ESR & Externally Supported Roof \\
\hline EV & Electric Vehicles \\
\hline FC & Fuel Cells \\
\hline FCEV & Fuel cell EV \\
\hline FL & Fuzzy Logic \\
\hline FS & Fuel Storage \\
\hline FSEV & Formula student electric vehicle \\
\hline GA & Genetic algorithm \\
\hline $\mathrm{GaN}$ & Gallium nitride \\
\hline G2V & Grid to vehicle \\
\hline HESS & Hybrid Energy Storage Systems \\
\hline $\mathrm{HEV}$ & Hybrid Electric Vehicle \\
\hline HV & Hybrid Vehicles \\
\hline HVDCPT & HVDC Power Transmission \\
\hline IF & Impact factor \\
\hline LVP & Land Vehicle Propulsion \\
\hline MIMO & Multiple-input-multiple-output \\
\hline MMC & modular multilevel converter \\
\hline PC & Power Converters \\
\hline PE & Power Electronics \\
\hline PEC & Power Electronics Converter \\
\hline PHEV & Plug-in Hybrid Vehicles \\
\hline PI & Proportional-Integral \\
\hline PL & Power loss \\
\hline PSO & Particle swarm optimization \\
\hline $\mathrm{RB}$ & Regenerative Braking \\
\hline RES & Renewable Energy Resources \\
\hline SB & Secondary Batteries \\
\hline SC & Supercapacitor \\
\hline $\mathrm{SiC}$ & Silicon carbide \\
\hline SM & Storage Management \\
\hline $\mathrm{SOC}$ & State of charge \\
\hline SOE & State of energy \\
\hline SRM & switched reluctance motor \\
\hline ST & Shoot-through \\
\hline THD & Total harmonic distortion \\
\hline $\mathrm{TM}$ & Traction Motors \\
\hline UC & Ultracapacitors \\
\hline UWBG & Ultra-wide bandgap \\
\hline VA & Vehicle Applications \\
\hline $\mathrm{VC}$ & Voltage Control \\
\hline VSI & Voltage source inverter \\
\hline V2G & Vehicle-to-grid \\
\hline WBG & Wide bandgap \\
\hline
\end{tabular}

\section{References}

1. Fritz, M.; Plötz, P.; Funke, S.A. The Impact of Ambitious Fuel Economy Standards on the Market Uptake of Electric Vehicles and Specific $\mathrm{CO}_{2}$ Emissions. Energy Policy 2019, 135, 111006. [CrossRef]

2. Singh, S.; Jain, S.; Ps, V.; Tiwari, A.K.; Nouni, M.R.; Pandey, J.K.; Goel, S. Hydrogen: A Sustainable Fuel for Future of the Transport Sector. Renew. Sustain. Energy Rev. 2015, 51, 623-633. [CrossRef]

3. Xing, L.-N.; Xu, H.-L.; Kardan Sani, A.; Hossain, A.; Muyeen, S.M.; Mcgordon, A.; Monteiro, V. Techno-Economic and Environmental Assessment of the Hybrid Energy System Considering Electric and Thermal Loads. Electronics 2021, $10,3136$. [CrossRef] 
4. Yao, Z.; Wang, Y.; Liu, B.; Zhao, B.; Jiang, Y. Fuel Consumption and Transportation Emissions Evaluation of Mixed Traffic Flow with Connected Automated Vehicles and Human-Driven Vehicles on Expressway. Energy 2021, 230, 120766. [CrossRef]

5. Ehsani, M.; Singh, K.V.; Bansal, H.O.; Mehrjardi, R.T. State of the Art and Trends in Electric and Hybrid Electric Vehicles. Proc. IEEE 2021, 109, 967-984. [CrossRef]

6. Quraan, M.; Yeo, T.; Tricoli, P. Design and Control of Modular Multilevel Converters for Battery Electric Vehicles. IEEE Trans. Power Electron. 2016, 31, 507-517. [CrossRef]

7. Ding, N.; Prasad, K.; Lie, T.T. The Electric Vehicle: A Review. Int. J. Electr. Hybrid Veh. 2017, 9, 49-66. [CrossRef]

8. How, D.N.T.; Hannan, M.A.; Lipu, M.S.H.; Sahari, K.S.M.; Ker, P.J.; Muttaqi, K.M. State-of-Charge Estimation of Li-Ion Battery in Electric Vehicles: A Deep Neural Network Approach. IEEE Trans. Ind. Appl. 2020, 56, 5565-5574. [CrossRef]

9. Beraki, M.W.; Trovao, J.P.F.; Perdigao, M.S.; Dubois, M.R. Variable Inductor Based Bidirectional DC-DC Converter for Electric Vehicles. IEEE Trans. Veh. Technol. 2017, 66, 8764-8772. [CrossRef]

10. Chan, C.C.; Chau, K.T. An Overview of Power Electronics in Electric Vehicles. IEEE Trans. Ind. Electron. 1997, 44, 3-13. [CrossRef]

11. Khan, M.A.; Ahmed, A.; Husain, I.; Sozer, Y.; Badawy, M. Performance Analysis of Bidirectional DC-DC Converters for Electric Vehicles. IEEE Trans. Ind. Appl. 2015, 51, 3442-3452. [CrossRef]

12. Chen, H.; Kim, H.; Erickson, R.; Maksimovic, D. Electrified Automotive Powertrain Architecture Using Composite DC-DC Converters. IEEE Trans. Power Electron. 2017, 32, 98-116. [CrossRef]

13. Sun, Q.; Wu, J.; Gan, C.; Si, J.; Guo, J.; Hu, Y. Cascaded Multiport Converter for SRM-Based Hybrid Electrical Vehicle Applications. IEEE Trans. Power Electron. 2019, 34, 11940-11951. [CrossRef]

14. Hannan, M.A.; Lipu, M.S.H.; Ker, P.J.; Begum, R.A.; Agelidis, V.G.; Blaabjerg, F. Power Electronics Contribution to Renewable Energy Conversion Addressing Emission Reduction: Applications, Issues, and Recommendations. Appl. Energy 2019, $251,113404$. [CrossRef]

15. Miri, I.; Fotouhi, A.; Ewin, N. Electric Vehicle Energy Consumption Modelling and Estimation-A Case Study. Int. J. Energy Res. 2021, 45, 501-520. [CrossRef]

16. Javorski Eckert, J.; Corrêa de Alkmin e Silva, L.; Mazzariol Santiciolli, F.; dos Santos Costa, E.; Corrêa, F.C.; Giuseppe Dedini, F. Energy Storage and Control Optimization for an Electric Vehicle. Int. J. Energy Res. 2018, 42, 3506-3523. [CrossRef]

17. Khan, M.M.H.; Hossain, A.; Ullah, A.; Lipu, M.S.H.; Shahnewaz Siddiquee, S.M.; Alam, M.S.; Jamal, T.; Ahmed, H. Integration of Large-Scale Electric Vehicles into Utility Grid: An Efficient Approach for Impact Analysis and Power Quality Assessment. Sustainability 2021, 13, 10943. [CrossRef]

18. Zhai, L.; Hu, G.; Lv, M.; Zhang, T.; Hou, R. Comparison of Two Design Methods of EMI Filter for High Voltage Power Supply in DC-DC Converter of Electric Vehicle. IEEE Access 2020, 8, 66564-66577. [CrossRef]

19. Gomez Navarro, F.J.; Yebra, L.J.; Gomez Medina, F.J.; Gimenez-Fernandez, A. DC-DC Linearized Converter Model for Faster Simulation of Lightweight Urban Electric Vehicles. IEEE Access 2020, 8, 85380-85394. [CrossRef]

20. Elsayad, N.; Moradisizkoohi, H.; Mohammed, O.A. A New Hybrid Structure of a Bidirectional DC-DC Converter with High Conversion Ratios for Electric Vehicles. IEEE Trans. Veh. Technol. 2020, 69, 194-206. [CrossRef]

21. Hannan, M.A.; Lipu, M.S.H.; Hussain, A.; Mohamed, A. A Review of Lithium-Ion Battery State of Charge Estimation and Management System in Electric Vehicle Applications: Challenges and Recommendations. Renew. Sustain. Energy Rev. 2017, 78, 834-854. [CrossRef]

22. Lei, J.; Zhou, B.; Wei, J.; Bian, J.; Zhu, Y.; Yu, J.; Yang, Y. Predictive Power Control of Matrix Converter with Active Damping Function. IEEE Trans. Ind. Electron. 2016, 63, 4550-4559. [CrossRef]

23. Thirumalaivasan, R.; Xu, Y.; Janaki, M. Power Control with Z-Source Converter Based Unified Power Flow Controller. IEEE Trans. Power Electron. 2017, 32, 9413-9423. [CrossRef]

24. Pedroso, M.D.; Nascimento, C.B.; Tusset, A.M.; Kaster, M.D.S. Performance Comparison between Nonlinear and Linear Controllers with Weighted Adaptive Control Applied to a Buck Converter Using Poles Placement Design. In Proceedings of the IEEE International Symposium on Industrial Electronics, Taipei, China, 28-31 May 2013; pp. 1-6.

25. Bhat, V.S.; Kumar, V.; Dayanand, N.; Shettigar, A.; Nikhitha, J. Comparative Study of PID Control Algorithms for an Electric Vehicle. In AIP Conference Proceedings; American Institute of Physics Inc.: Melville, NY, USA, 2020; Volume 2236, pp. 1-6.

26. Babaei, F.; Lashkari, Z.B.; Safari, A.; Farrokhifar, M.; Salehi, J. Salp Swarm Algorithm-Based Fractional-Order PID Controller for LFC Systems in the Presence of Delayed EV Aggregators. IET Electr. Syst. Transp. 2020, 10, 259-267. [CrossRef]

27. Hannan, M.A.; Ali, J.A.; Ker, P.J.; Mohamed, A.; Lipu, M.S.H.; Hussain, A. Switching Techniques and Intelligent Controllers for Induction Motor Drive: Issues and Recommendations. IEEE Access 2018, 6, 47489-47510. [CrossRef]

28. Parra, A.; Zubizarreta, A.; Pérez, J.; Dendaluce, M. Intelligent Torque Vectoring Approach for Electric Vehicles with Per-Wheel Motors. Complexity 2018, 2018, 7030184. [CrossRef]

29. Hassan, S.; Kamal, T.; Riaz, M.; Shah, S.; Ali, H.; Riaz, M.; Sarmad, M.; Zahoor, A.; Khan, M.; Miqueleiz, J. Intelligent Control of Wind-Assisted PHEVs Smart Charging Station. Energies 2019, 12, 909. [CrossRef]

30. Pérez-Pimentel, Y.; Osuna-Galán, I.; Avilés-Cruz, C.; Villegas-Cortez, J. Power Supply Management for an Electric Vehicle Using Fuzzy Logic. Appl. Comput. Intell. Soft Comput. 2018, 2018, 2846748. [CrossRef]

31. Yao, E.; Liu, T.; Lu, T.; Yang, Y. Optimization of Electric Vehicle Scheduling with Multiple Vehicle Types in Public Transport. Sustain. Cities Soc. 2020, 52, 101862. [CrossRef] 
32. Shen, Z.J.M.; Feng, B.; Mao, C.; Ran, L. Optimization Models for Electric Vehicle Service Operations: A Literature Review. Transp. Res. Part B Methodol. 2019, 128, 462-477. [CrossRef]

33. Ansari, S.; Ayob, A.; Lipu, M.S.H.; Hussain, A.; Saad, M.H.M. Multi-Channel Profile Based Artificial Neural Network Approach for Remaining Useful Life Prediction of Electric Vehicle Lithium-Ion Batteries. Energies 2021, 14, 7521. [CrossRef]

34. Mumtaz, F.; Yahaya, N.Z.; Meraj, S.T.; Singh, B.; Kannan, R.; Ibrahim, O. Review on Non-Isolated DC-DC Converters and Their Control Techniques for Renewable Energy Applications. Ain Shams Eng. J. 2021, 12, 3747-3763. [CrossRef]

35. Aymen, F.; Mahmoudi, C. A Novel Energy Optimization Approach for Electrical Vehicles in a Smart City. Energies 2019, 12, 929. [CrossRef]

36. Akkaldevi, C.; Chitta, S.D.; Jaidi, J.; Panchal, S.; Fowler, M.; Fraser, R. Coupled Electrochemical-Thermal Simulations and Validation of Minichannel Cold-Plate Water-Cooled Prismatic 20 Ah LiFePO4 Battery. Electrochem 2021, 2, 643-663. [CrossRef]

37. Purohit, K.; Srivastava, S.; Nookala, V.; Joshi, V.; Shah, P.; Sekhar, R.; Panchal, S.; Fowler, M.; Fraser, R.; Tran, M.K.; et al. Soft Sensors for State of Charge, State of Energy, and Power Loss in Formula Student Electric Vehicle. Appl. Syst. Innov. 2021, 4, 78. [CrossRef]

38. Choudhari, V.G.; Dhoble, A.S.; Panchal, S.; Fowler, M.; Fraser, R. Numerical Investigation on Thermal Behaviour of $5 \times 5$ Cell Configured Battery Pack Using Phase Change Material and Fin Structure Layout. J. Energy Storage 2021, 43, 103234. [CrossRef]

39. Zhu, C.; Han, J.; Zhang, H.; Lu, F.; Liu, K.; Zhang, X. Modeling and Control of An Integrated Self-Heater for Automotive Batteries Based on Traction Motor Drive Reconfiguration. IEEE J. Emerg. Sel. Top. Power Electron. 2021, 1. [CrossRef]

40. Liu, K.; Yang, Z.; Tang, X.; Cao, W. Automotive Battery Equalizers Based on Joint Switched-Capacitor and Buck-Boost Converters. IEEE Trans. Veh. Technol. 2020, 69, 12716-12724. [CrossRef]

41. Shang, Y.; Liu, K.; Cui, N.; Wang, N.; Li, K.; Zhang, C. A Compact Resonant Switched-Capacitor Heater for Lithium-Ion Battery Self-Heating at Low Temperatures. IEEE Trans. Power Electron. 2020, 35, 7134-7144. [CrossRef]

42. Shang, Y.; Liu, K.; Cui, N.; Zhang, Q.; Zhang, C. A Sine-Wave Heating Circuit for Automotive Battery Self-Heating at Subzero Temperatures. IEEE Trans. Ind. Inform. 2020, 16, 3355-3365. [CrossRef]

43. Liu, K.; Hu, X.; Zhou, H.; Tong, L.; Widanage, W.D.; Marco, J. Feature Analyses and Modeling of Lithium-Ion Battery Manufacturing Based on Random Forest Classification. IEEE/ASME Trans. Mechatron. 2021, 26, 2944-2955. [CrossRef]

44. Liu, K.; Hu, X.; Meng, J.; Guerrero, J.M.; Teodorescu, R. RUBoost-Based Ensemble Machine Learning for Electrode Quality Classification in Li-Ion Battery Manufacturing. IEEE/ASME Trans. Mechatron. 2021, 1-10. [CrossRef]

45. Chakraborty, S.; Vu, H.N.; Hasan, M.M.; Tran, D.D.; El Baghdadi, M.; Hegazy, O. DC-DC Converter Topologies for Electric Vehicles, Plug-in Hybrid Electric Vehicles and Fast Charging Stations: State of the Art and Future Trends. Energies 2019, 12, 1569. [CrossRef]

46. Anbazhagan, L.; Ramiah, J.; Krishnaswamy, V.; Jayachandran, D.N. A Comprehensive Review on Bidirectional Traction Converter for Electric Vehicles. Int. J. Electron. Telecommun. 2019, 65, 635-649.

47. Jagadeesh, I.; Indragandhi, V. Review and Comparative Analysis on Dc-Dc Converters Used in Electric Vehicle Applications. IOP Conf. Ser. Mater. Sci. Eng. 2019, 623, 012005. [CrossRef]

48. Reddy, K.J.; Natarajan, S. Energy Sources and Multi-Input DC-DC Converters Used in Hybrid Electric Vehicle Applications-A Review. Int. J. Hydrog. Energy 2018, 43, 17387-17408. [CrossRef]

49. Reddy, J.; Sudhakar, N. High Voltage Gain Switched Capacitor Boost Converter with ANFIS Controller for Fuel Cell Electric Vehicle Applications. In Proceedings of the 2018 2nd IEEE International Conference on Power Electronics, Intelligent Control and Energy Systems (ICPEICES), Delhi, India, 22-24 October 2018; IEEE: Piscataway, NJ, USA; pp. 465-470. [CrossRef]

50. Reddy, K.J.; Sudhakar, N. High Voltage Gain Interleaved Boost Converter with Neural Network Based Mppt Controller for Fuel Cell Based Electric Vehicle Applications. IEEE Access 2017, 6, 3899-3908. [CrossRef]

51. Affam, A.; Buswig, Y.M.; Othman, A.-K.B.H.; Julai, N.B.; Qays, O. A Review of Multiple Input DC-DC Converter Topologies Linked with Hybrid Electric Vehicles and Renewable Energy Systems. Renew. Sustain. Energy Rev. 2021, 135, 110186. [CrossRef]

52. Rehman, Z.; Al-Bahadly, I.; Mukhopadhyay, S. Multiinput DC-DC Converters in Renewable Energy Applications-An Overview. Renew. Sustain. Energy Rev. 2015, 41, 521-539. [CrossRef]

53. Turksoy, A.; Teke, A.; Alkaya, A. A Comprehensive Overview of the Dc-Dc Converter-Based Battery Charge Balancing Methods in Electric Vehicles. Renew. Sustain. Energy Rev. 2020, 133, 110274. [CrossRef]

54. Bellur, D.M.; Kazimierczuk, M.K. DC-DC Converters for Electric Vehicle Applications. In Proceedings of the 2007 Electrical Insulation Conference and Electrical Manufacturing Expo, Nashville, TN, USA, 22-24 October 2007; pp. 286-293. [CrossRef]

55. Mounica, V.; Obulesu, Y.P. A Comprehensive Review on Non-Isolated Bidirectional DC-DC Converter Topologies for Electric Vehicle Application. In Advances in Automation, Signal Processing, Instrumentation, and Control; Springer: Singapore, 2021; Volume 700, pp. 2097-2108.

56. Eroğlu, F.; Kurtoğlu, M.; Vural, A.M. Bidirectional DC-DC Converter Based Multilevel Battery Storage Systems for Electric Vehicle and Large-Scale Grid Applications: A Critical Review Considering Different Topologies, State-of-Charge Balancing and Future Trends. IET Renew. Power Gener. 2021, 15, 915-938. [CrossRef]

57. Bortoluzzi, M.; Correia de Souza, C.; Furlan, M. Bibliometric Analysis of Renewable Energy Types Using Key Performance Indicators and Multicriteria Decision Models. Renew. Sustain. Energy Rev. 2021, 143, 110958. [CrossRef]

58. Choi, W.; Kim, J.; Lee, S.E.; Park, E. Smart Home and Internet of Things: A Bibliometric Study. J. Clean. Prod. 2021, $301,126908$. [CrossRef] 
59. Tseng, M.L.; Chang, C.H.; Lin, C.W.R.; Wu, K.J.; Chen, Q.; Xia, L.; Xue, B. Future Trends and Guidance for the Triple Bottom Line and Sustainability: A Data Driven Bibliometric Analysis. Environ. Sci. Pollut. Res. 2020, 27, 33543-33567. [CrossRef]

60. Ismail, S.A.; Ang, W.L.; Mohammad, A.W. Electro-Fenton Technology for Wastewater Treatment: A Bibliometric Analysis of Current Research Trends, Future Perspectives and Energy Consumption Analysis. J. Water Process Eng. 2021, $40,101952$. [CrossRef]

61. Gingras, Y. Bibliometrics and Research Evaluation: Uses and Abuses; The MIT Press: Cambridge, MA, USA, 2016; ISBN 978-0262035125.

62. Andrés, A. Measuring Academic Research: How to Undertake a Bibliometric Study; Chandos Publishing: Oxford, UK, 2009; ISBN 978-1-84334-528-2.

63. Zhao, X.; Wang, S.; Wang, X. Characteristics and Trends of Research on New Energy Vehicle Reliability Based on the Web of Science. Sustainability 2018, 10, 3560. [CrossRef]

64. Arriola, E.R.; Ubando, A.T.; Chen, W.H. A Bibliometric Review on the Application of Fuzzy Optimization to Sustainable Energy Technologies. Int. J. Energy Res. 2020, 46, 6-27. [CrossRef]

65. Gandia, R.M.; Antonialli, F.; Cavazza, B.H.; Neto, A.M.; de Lima, D.A.; Sugano, J.Y.; Nicolai, I.; Zambalde, A.L. Autonomous Vehicles: Scientometric and Bibliometric Review*. Transp. Rev. 2019, 39, 9-28. [CrossRef]

66. Miah, M.S.; Shahadat, M.; Lipu, H.; Meraj, T.; Hasan, K.; Ansari, S.; Jamal, T.; Masrur, H.; Elavarasan, R.M.; Hussain, A.; et al. Optimized Energy Management Schemes for Electric Vehicle Applications: A Bibliometric Analysis towards Future Trends. Sustainability 2021, 13, 12800. [CrossRef]

67. Wang, S.; Yu, J. A Bibliometric Research on Next-Generation Vehicles Using CiteSpace. Recycling 2021, 6, 14. [CrossRef]

68. Zhang, L.; Liu, Y.; Pang, B. China's Development on New Energy Vehicle Battery Industry: Based on Market and Bibliometrics. In IOP Conference Series: Earth and Environmental Science; IOP Publishing: Bristol, UK, 2020; Volume 581, pp. 1-6.

69. Cabeza, L.F.; Chàfer, M.; Mata, É. Comparative Analysis of Web of Science and Scopus on the Energy Efficiency and Climate Impact of Buildings. Energies 2020, 13, 409. [CrossRef]

70. Borri, E.; Tafone, A.; Zsembinszki, G.; Comodi, G.; Romagnoli, A.; Cabeza, L.F. Recent Trends on Liquid Air Energy Storage: A Bibliometric Analysis. Appl. Sci. 2020, 10, 2773. [CrossRef]

71. Katnapally, A.; Manthati, U.B.; Raveendran, A.C.; Punna, S. A Predictive Power Management Scheme for Hybrid Energy Storage System in Electric Vehicle. Int. J. Circuit Theory Appl. 2021, 49, 3864-3878. [CrossRef]

72. Fang, J.; Deng, H.; Tashakor, N.; Blaabjerg, F.; Goetz, S.M. State-Space Modeling and Control of Grid-Tied Power Converters with Capacitive/Battery Energy Storage and Grid-Supportive Services. IEEE J. Emerg. Sel. Top. Power Electron. 2021, 1. [CrossRef]

73. Sorlei, I.S.; Bizon, N.; Thounthong, P.; Varlam, M.; Carcadea, E.; Culcer, M.; Iliescu, M.; Raceanu, M. Fuel Cell Electric Vehicles-A Brief Review of Current Topologies and Energy Management Strategies. Energies 2021, 14, 252. [CrossRef]

74. Moradisizkoohi, H.; Elsayad, N.; Mohammed, O.A. A Voltage-Quadrupler Interleaved Bidirectional DC-DC Converter with Intrinsic Equal Current Sharing Characteristic for Electric Vehicles. IEEE Trans. Ind. Electron. 2021, 68, 1803-1813. [CrossRef]

75. Lionel, F.D.J.; Dias, J.; Krishna Srinivasan, M.; Parandhaman, B.; Prabhakaran, P. A Novel Non-Isolated Dual-Input DC-DC Boost Converter for Hybrid Electric Vehicle Application. Int. J. Emerg. Electr. Power Syst. 2021, 22, 191-204. [CrossRef]

76. Zhang, L.; Hu, X.; Wang, Z.; Ruan, J.; Ma, C.; Song, Z.; Dorrell, D.G.; Pecht, M.G. Hybrid Electrochemical Energy Storage Systems: An Overview for Smart Grid and Electrified Vehicle Applications. Renew. Sustain. Energy Rev. 2021, 139, 110581. [CrossRef]

77. Fang, Z.; Wei, Z.; Huang, Z.; Liu, F. Onboard Energy Storage System Based on Interleaved High-Conversion-Ratio Quasi-Resonant Converter with Small Characteristic Impedance. IEEE Trans. Veh. Technol. 2021, 70, 4238-4251. [CrossRef]

78. Chelladurai, B.; Sundarabalan, C.K.; Santhanam, S.N.; Guerrero, J.M. Interval Type-2 Fuzzy Logic Controlled Shunt Converter Coupled Novel High-Quality Charging Scheme for Electric Vehicles. IEEE Trans. Ind. Inform. 2021, 17, 6084-6093. [CrossRef]

79. Raman, S.R.; Cheng, K.W.; Xue, X.D.; Fong, Y.C.; Cheung, S. Hybrid Energy Storage System with Vehicle Body Integrated Super-Capacitor and Li-Ion Battery: Model, Design and Implementation, for Distributed Energy Storage. Energies 2021, 14, 6553. [CrossRef]

80. Shi, R.; Semsar, S.; Lehn, P.W. Single-Stage Hybrid Energy Storage Integration in Electric Vehicles Using Vector Controlled Power Sharing. IEEE Trans. Ind. Electron. 2021, 68, 10623-10633. [CrossRef]

81. Cao, J.; Emadi, A. A New Battery/Ultracapacitor Hybrid Energy Storage System for Electric, Hybrid, and Plug-in Hybrid Electric Vehicles. IEEE Trans. Power Electron. 2012, 27, 122-132. [CrossRef]

82. Song, Z.; Li, J.; Han, X.; Xu, L.; Lu, L.; Ouyang, M.; Hofmann, H. Multi-Objective Optimization of a Semi-Active Battery/Supercapacitor Energy Storage System for Electric Vehicles. Appl. Energy 2014, 135, 212-224. [CrossRef]

83. Zheng, Z.; Wang, K.; Xu, L.; Li, Y. A Hybrid Cascaded Multilevel Converter for Battery Energy Management Applied in Electric Vehicles. IEEE Trans. Power Electron. 2014, 29, 3537-3546. [CrossRef]

84. Nahavandi, A.; Hagh, M.T.; Sharifian, M.B.B.; Danyali, S. A Nonisolated Multiinput Multioutput DC-DC Boost Converter for Electric Vehicle Applications. IEEE Trans. Power Electron. 2015, 30, 1818-1835. [CrossRef]

85. Laldin, O.; Moshirvaziri, M.; Trescases, O. Predictive Algorithm for Optimizing Power Flow in Hybrid Ultracapacitor/Battery Storage Systems for Light Electric Vehicles. IEEE Trans. Power Electron. 2013, 28, 3882-3895. [CrossRef]

86. Samosir, A.S.; Yatim, A.H.M. Implementation of Dynamic Evolution Control of Bidirectional DC-DC Converter for Interfacing Ultracapacitor Energy Storage to Fuel-Cell System. IEEE Trans. Ind. Electron. 2010, 57, 3468-3473. [CrossRef]

87. El Fadil, H.; Giri, F.; Guerrero, J.M.; Tahri, A. Modeling and Nonlinear Control of a Fuel Cell/Supercapacitor Hybrid Energy Storage System for Electric Vehicles. IEEE Trans. Veh. Technol. 2014, 63, 3011-3018. [CrossRef] 
88. Blanes, J.M.; Gutiérrez, R.; Garrigós, A.; Lizán, J.L.; Cuadrado, J.M. Electric Vehicle Battery Life Extension Using Ultracapacitors and an FPGA Controlled Interleaved Buck-Boost Converter. IEEE Trans. Power Electron. 2013, 28, 5940-5948. [CrossRef]

89. Song, Z.; Hou, J.; Hofmann, H.; Li, J.; Ouyang, M. Sliding-Mode and Lyapunov Function-Based Control for Battery/Supercapacitor Hybrid Energy Storage System Used in Electric Vehicles. Energy 2017, 122, 601-612. [CrossRef]

90. Akar, F.; Tavlasoglu, Y.; Ugur, E.; Vural, B.; Aksoy, I. A Bidirectional Nonisolated Multi-Input DC-DC Converter for Hybrid Energy Storage Systems in Electric Vehicles. IEEE Trans. Veh. Technol. 2016, 65, 7944-7955. [CrossRef]

91. Rubino, L.; Capasso, C.; Veneri, O. Review on Plug-in Electric Vehicle Charging Architectures Integrated with Distributed Energy Sources for Sustainable Mobility. Appl. Energy 2017, 207, 438-464. [CrossRef]

92. Wang, B.; Xu, J.; Cao, B.; Zhou, X. A Novel Multimode Hybrid Energy Storage System and Its Energy Management Strategy for Electric Vehicles. J. Power Sources 2015, 281, 432-443. [CrossRef]

93. Ahrabi, R.R.; Ardi, H.; Elmi, M.; Ajami, A. A Novel Step-Up Multiinput DC-DC Converter for Hybrid Electric Vehicles Application. IEEE Trans. Power Electron. 2017, 32, 3549-3561. [CrossRef]

94. Rivera, S.; Wu, B. Electric Vehicle Charging Station with an Energy Storage Stage for Split-DC Bus Voltage Balancing. IEEE Trans. Power Electron. 2017, 32, 2376-2386. [CrossRef]

95. Wang, B.; Xu, J.; Wai, R.J.; Cao, B. Adaptive Sliding-Mode with Hysteresis Control Strategy for Simple Multimode Hybrid Energy Storage System in Electric Vehicles. IEEE Trans. Ind. Electron. 2017, 64, 1404-1414. [CrossRef]

96. Varajao, D.; Araujo, R.E.; Miranda, L.M.; Lopes, J.A.P. Modulation Strategy for a Single-Stage Bidirectional and Isolated AC-DC Matrix Converter for Energy Storage Systems. IEEE Trans. Ind. Electron. 2018, 65, 3458-3468. [CrossRef]

97. Capasso, C.; Veneri, O. Experimental Study of a DC Charging Station for Full Electric and Plug in Hybrid Vehicles. Appl. Energy 2015, 152, 131-142. [CrossRef]

98. Amjadi, Z.; Williamson, S.S. A Novel Control Technique for a Switched-Capacitor-Converter-Based Hybrid Electric Vehicle Energy Storage System. IEEE Trans. Ind. Electron. 2010, 57, 926-934. [CrossRef]

99. Lai, C.M.; Cheng, Y.H.; Hsieh, M.H.; Lin, Y.C. Development of a Bidirectional DC/DC Converter with Dual-Battery Energy Storage for Hybrid Electric Vehicle System. IEEE Trans. Veh. Technol. 2018, 67, 1036-1052. [CrossRef]

100. Sun, B.; Dragičević, T.; Freijedo, F.D.; Vasquez, J.C.; Guerrero, J.M. A Control Algorithm for Electric Vehicle Fast Charging Stations Equipped with Flywheel Energy Storage Systems. IEEE Trans. Power Electron. 2016, 31, 6674-6685. [CrossRef]

101. Reddy, K.J.; Sudhakar, N.; Saravanan, S.; Babu, B.C. High step-up boost converter with neural network based MPPT controller for a PEMFC power source used in vehicular applications. Int. J. Emerg. Electr. Power Syst. 2018, 19. [CrossRef]

102. Geetha, A.; Subramani, C. A Comprehensive Review on Energy Management Strategies of Hybrid Energy Storage System for Electric Vehicles. Int. J. Energy Res. 2017, 41, 1817-1834. [CrossRef]

103. Amjadi, Z.; Williamson, S.S. Prototype Design and Controller Implementation for a Battery-Ultracapacitor Hybrid Electric Vehicle Energy Storage System. IEEE Trans. Smart Grid 2012, 3, 332-340. [CrossRef]

104. Kasimalla, V.K.; Srinivasulu, G.; Velisala, V. A Review on Energy Allocation of Fuel Cell/Battery/Ultracapacitor for Hybrid Electric Vehicles. Int. J. Energy Res. 2018, 42, 4263-4283. [CrossRef]

105. Gan, C.; Sun, Q.; Wu, J.; Kong, W.; Shi, C.; Hu, Y. MMC-Based SRM Drives with Decentralized Battery Energy Storage System for Hybrid Electric Vehicles. IEEE Trans. Power Electron. 2019, 34, 2608-2621. [CrossRef]

106. Veneri, O.; Capasso, C.; Patalano, S. Experimental Investigation into the Effectiveness of a Super-Capacitor Based Hybrid Energy Storage System for Urban Commercial Vehicles. Appl. Energy 2018, 227, 312-323. [CrossRef]

107. Pires, V.F.; Cordeiro, A.; Foito, D.; Silva, J.F. High Step-up DC-DC Converter for Fuel Cell Vehicles Based on Merged Quadratic Boost-Cuk. IEEE Trans. Veh. Technol. 2019, 68, 7521-7530. [CrossRef]

108. Zhang, Q.; Li, G. Experimental Study on a Semi-Active Battery-Supercapacitor Hybrid Energy Storage System for Electric Vehicle Application. IEEE Trans. Power Electron. 2020, 35, 1014-1021. [CrossRef]

109. Jiang, J.; Bao, Y.; Wang, L.Y. Topology of a Bidirectional Converter for Energy Interaction between Electric Vehicles and the Grid. Energies 2014, 7, 4858-4894. [CrossRef]

110. Wang, C.; Xiong, R.; He, H.; Ding, X.; Shen, W. Efficiency analysis of a bidirectional DC/DC converter in a hybrid energy storage system for plug-in hybrid electric vehicles. Appl. Energy 2016, 183, 612-622. [CrossRef]

111. Muttaqi, K.M.; Islam, M.R.; Sutanto, D. Future Power Distribution Grids: Integration of Renewable Energy, Energy Storage, Electric Vehicles, Superconductor, and Magnetic Bus. IEEE Trans. Appl. Supercond. 2019, 29, 3800305. [CrossRef]

112. Wen, H.; Su, B. Hybrid-Mode Interleaved Boost Converter Design for Fuel Cell Electric Vehicles. Energy Convers. Manag. 2016, 122, 477-487. [CrossRef]

113. Kandasamy, K.; Vilathgamuwa, M.; Tseng, K.J. Inter-Module State-of-Charge Balancing and Fault-Tolerant Operation of Cascaded H-Bridge Converter Using Multi-Dimensional Modulation for Electric Vehicle Application. IET Power Electron. 2015, 8, 1912-1919. [CrossRef]

114. Mukherjee, N.; Strickland, D. Analysis and Comparative Study of Different Converter Modes in Modular Second-Life Hybrid Battery Energy Storage Systems. IEEE J. Emerg. Sel. Top. Power Electron. 2016, 4, 547-563. [CrossRef]

115. Wang, B.; Xu, J.; Xu, D.; Yan, Z. Implementation of an Estimator-Based Adaptive Sliding Mode Control Strategy for a Boost Converter Based Battery/Supercapacitor Hybrid Energy Storage System in Electric Vehicles. Energy Convers. Manag. 2017, 151, 562-572. [CrossRef] 
116. Amjadi, Z.; Williamson, S.S. Digital Control of a Bidirectional DC/DC Switched Capacitor Converter for Hybrid Electric Vehicle Energy Storage System Applications. IEEE Trans. Smart Grid 2014, 5, 158-166. [CrossRef]

117. Zhang, Y.; Cheng, X.F.; Yin, C.; Cheng, S. A Soft-Switching Bidirectional DC-DC Converter for the Battery Super-Capacitor Hybrid Energy Storage System. IEEE Trans. Ind. Electron. 2018, 65, 7856-7865. [CrossRef]

118. Badawy, M.O.; Husain, T.; Sozer, Y.; de Abreu-Garcia, J.A. Integrated Control of an IPM Motor Drive and a Novel Hybrid Energy Storage System for Electric Vehicles. IEEE Trans. Ind. Appl. 2017, 53, 5810-5819. [CrossRef]

119. Michalczuk, M.; Ufnalski, B.; Grzesiak, L.M. Fuzzy Logic Based Power Management Strategy Using Topographic Data for an Electric Vehicle with a Battery-Ultracapacitor Energy Storage. COMPEL Int. J. Comput. Math. Electr. Electron. Eng. 2015, 34, 173-188. [CrossRef]

120. Lipu, M.H.; Hannan, M.A.; Karim, T.F.; Hussain, A.; Saad, M.H.M.; Ayob, A.; Miah, M.S.; Indra Mahlia, T.M. Intelligent Algorithms and Control Strategies for Battery Management System in Electric Vehicles: Progress, Challenges and Future Outlook. J. Clean. Prod. 2021, 292, 126044. [CrossRef]

121. Yang, B.; Wang, J.; Zhang, X.; Wang, J.; Shu, H.; Li, S.; He, T.; Lan, C.; Yu, T. Applications of Battery/Supercapacitor Hybrid Energy Storage Systems for Electric Vehicles Using Perturbation Observer Based Robust Control. J. Power Sources 2020, 448, 227444. [CrossRef]

122. Amjadi, Z.; Williamson, S.S. Modeling, Simulation, and Control of an Advanced Luo Converter for Plug-in Hybrid Electric Vehicle Energy-Storage System. IEEE Trans. Veh. Technol. 2011, 60, 64-75. [CrossRef]

123. İnci, M.; Büyük, M.; Demir, M.H.; İlbey, G. A Review and Research on Fuel Cell Electric Vehicles: Topologies, Power Electronic Converters, Energy Management Methods, Technical Challenges, Marketing and Future Aspects. Renew. Sustain. Energy Rev. 2021, 137, 110648. [CrossRef]

124. Khan, S.A.; Islam, M.R.; Guo, Y.; Zhu, J. A New Isolated Multi-Port Converter with Multi-Directional Power Flow Capabilities for Smart Electric Vehicle Charging Stations. IEEE Trans. Appl. Supercond. 2019, 29, 0602504. [CrossRef]

125. Capasso, C.; Lauria, D.; Veneri, O. Experimental Evaluation of Model-Based Control Strategies of Sodium-Nickel Chloride Battery plus Supercapacitor Hybrid Storage Systems for Urban Electric Vehicles. Appl. Energy 2018, 228, 2478-2489. [CrossRef]

126. Zhang, Q.; Deng, W.; Zhang, S.; Wu, J. A Rule Based Energy Management System of Experimental Battery/Supercapacitor Hybrid Energy Storage System for Electric Vehicles. J. Control Sci. Eng. 2016, 2016, 6828269. [CrossRef]

127. Wang, K.; Wang, W.; Wang, L.; Li, L. An Improved SOC Control Strategy for Electric Vehicle Hybrid Energy Storage Systems. Energies 2020, 13, 5297. [CrossRef]

128. Li, Y.; Huang, X.; Liu, D.; Wang, M.; Xu, J. Hybrid Energy Storage System and Energy Distribution Strategy for Four-Wheel Independent-Drive Electric Vehicles. J. Clean. Prod. 2019, 220, 756-770. [CrossRef]

129. Wang, B.; Xu, J.; Cao, B.; Li, Q.; Yang, Q. Compound-Type Hybrid Energy Storage System and Its Mode Control Strategy for Electric Vehicles. J. Power Electron. 2015, 15, 849-859. [CrossRef]

130. Malik, M.Z.; Chen, H.; Nazir, M.S.; Khan, I.A.; Abdalla, A.N.; Ali, A.; Chen, W. A New Efficient Step-Up Boost Converter with CLD Cell for Electric Vehicle and New Energy Systems. Energies 2020, 13, 1791. [CrossRef]

131. Mumtaz, S.; Ali, S.; Ahmad, S.; Khan, L.; Hassan, S.Z.; Kamal, T. Energy Management and Control of Plug-In Hybrid Electric Vehicle Charging Stations in a Grid-Connected Hybrid Power System. Energies 2017, 10, 1923. [CrossRef]

132. Kumar, D.; Nema, R.K.; Gupta, S. A Comparative Review on Power Conversion Topologies and Energy Storage System for Electric Vehicles. Int. J. Energy Res. 2020, 44, 7863-7885. [CrossRef]

133. Omran, K.C.; Mosallanejad, A. SMES/Battery Hybrid Energy Storage System Based on Bidirectional Z-Source Inverter for Electric Vehicles. IET Electr. Syst. Transp. 2018, 8, 215-220. [CrossRef]

134. Chung, S.; Trescases, O. Hybrid Energy Storage System with Active Power-Mix Control in a Dual-Chemistry Battery Pack for Light Electric Vehicles. IEEE Trans. Transp. Electrif. 2017, 3, 600-617. [CrossRef]

135. Trovão, J.P.; Silva, M.A.; Antunes, C.H.; Dubois, M.R. Stability Enhancement of the Motor Drive DC Input Voltage of an Electric Vehicle Using On-Board Hybrid Energy Storage Systems. Appl. Energy 2017, 205, 244-259. [CrossRef]

136. Jha, M.; Blaabjerg, F.; Khan, M.A.; Kurukuru, V.S.B.; Haque, A. Intelligent Control of Converter for Electric Vehicles Charging Station. Energies 2019, 12, 2334. [CrossRef]

137. Xia, X.; Zhao, X.; Zeng, H.; Zeng, X. A Novel Design of Hybrid Energy Storage System for Electric Vehicles. Chin. J. Electr. Eng. 2018, 4, 45-51. [CrossRef]

138. Becker, J.; Schaeper, C.; Rothgang, S.; Sauer, D.U. Development and Validation of an Energy Management System for an Electric Vehicle with a Split Battery Storage System. J. Electr. Eng. Technol. 2013, 8, 920-929. [CrossRef]

139. Wang, B.; Zhang, X.; Manandhar, U.; Gooi, H.B.; Liu, Y.; Tan, X. Bidirectional Three-Level Cascaded Converter with Deadbeat Control for HESS in Solar-Assisted Electric Vehicles. IEEE Trans. Transp. Electrif. 2019, 5, 1190-1201. [CrossRef]

140. Liu, S.; Xie, X.; Yang, L. Analysis, Modeling and Implementation of a Switching Bi-Directional Buck-Boost Converter Based on Electric Vehicle Hybrid Energy Storage for V2G System. IEEE Access 2020, 8, 65868-65879. [CrossRef]

141. Wang, C.; Yang, R.; Yu, Q. Wavelet Transform Based Energy Management Strategies for Plug-in Hybrid Electric Vehicles Considering Temperature Uncertainty. Appl. Energy 2019, 256, 113928. [CrossRef]

142. Liu, Y.; Li, Y.; Liang, H.; He, J.; Cui, H. Energy Routing Control Strategy for Integrated Microgrids Including Photovoltaic, Battery-Energy Storage and Electric Vehicles. Energies 2019, 12, 302. [CrossRef] 
143. Cheng, T.; Lu, D.D.C. Three-Port Converters with a Flexible Power Flow for Integrating PV and Energy Storage into a DC Bus. J. Power Electron. 2017, 17, 1433-1444. [CrossRef]

144. Moradisizkoohi, H.; Elsayad, N.; Mohammed, O.A. Experimental Verification of a Double-Input Soft-Switched DC-DC Converter for Fuel Cell Electric Vehicle with Hybrid Energy Storage System. IEEE Trans. Ind. Appl. 2019, 55, 6451-6465. [CrossRef]

145. Bae, S.H.; Park, J.W. A Study on Optimal Operation Strategy for Mild Hybrid Electric Vehicle Based on Hybrid Energy Storage System. J. Electr. Eng. Technol. 2018, 13, 631-636. [CrossRef]

146. Bindu, R.; Thale, S. Power Management Strategy for an Electric Vehicle Driven by Hybrid Energy Storage System. IETE J. Res. 2020, 1-11. [CrossRef]

147. Wang, Z.; Wang, P.; Bi, H.; Qiu, M. A Bidirectional DC/DC Converter with Wide-Voltage Gain Range and Low-Voltage Stress for Hybrid-Energy Storage Systems in Electric Vehicles. J. Power Electron. 2020, 20, 76-86. [CrossRef]

148. Bai, Z.; Yan, Z.; Wu, X.; Xu, J.; Cao, B. Hळ Control for Battery/Supercapacitor Hybrid Energy Storage System Used in Electric Vehicles. Int. J. Automot. Technol. 2019, 20, 1287-1296. [CrossRef]

149. Vidhya, S.D.; Balaji, M. Modelling, Design and Control of a Light Electric Vehicle with Hybrid Energy Storage System for Indian Driving Cycle. Meas. Control 2019, 52, 1420-1433. [CrossRef]

150. De Melo, R.R.; Tofoli, F.L.; Daher, S.; Antunes, F.L.M. Interleaved Bidirectional DC-DC Converter for Electric Vehicle Applications Based on Multiple Energy Storage Devices. Electr. Eng. 2020, 102, 2011-2023. [CrossRef]

151. Veerendra, A.S.; Mohamed, M.R.; Sulaiman, M.H.; Sudhakar, K.; Peddakapu, K. Modelling and Simulation of Dual Sourced Front-End Converter for Hybrid Electric Vehicles. Int. J. Ambient Energy 2020, 1-8. [CrossRef]

152. Joseph, P.K.; Devaraj, E. Design of Hybrid Forward Boost Converter for Renewable Energy Powered Electric Vehicle Charging Applications. IET Power Electron. 2019, 12, 2015-2021. [CrossRef]

153. Zhang, X.; Yin, C.; Bai, H. Fixed-Boundary-Layer Sliding-Mode and Variable Switching Frequency Control for a Bidirectional DC-DC Converter in Hybrid Energy Storage System. Electr. Power Components Syst. 2017, 45, 1474-1485. [CrossRef]

154. Jiang, L.; Mi, C.; Li, S.; Yin, C. A Control Method to Improve the Efficiency of a Softswitching Non-Isolated Bidirectional DC-DC Converter for Hybrid and Plug-in Electric Vehicle Applications. Int. J. Power Electron. 2014, 6, 66-87. [CrossRef]

155. Rahman, A.U.; Zehra, S.S.; Ahmad, I.; Armghan, H. Fuzzy Supertwisting Sliding Mode-Based Energy Management and Control of Hybrid Energy Storage System in Electric Vehicle Considering Fuel Economy. J. Energy Storage 2021, 37, 102468. [CrossRef]

156. Suresh, K.; Bharatiraja, C.; Chellammal, N.; Tariq, M.; Chakrabortty, R.K.; Ryan, M.J.; Alamri, B. A Multifunctional Non-Isolated Dual Input-Dual Output Converter for Electric Vehicle Applications. IEEE Access 2021, 9, 64445-64460. [CrossRef]

157. Tseng, K.C.; Chang, Y.C.; Cheng, C.A. Implementation and Analysis of Ultracapacitor Charger in Hybrid Energy-Storage System for Electric-Vehicle Applications. IET Power Electron. 2020, 13, 1858-1864. [CrossRef]

158. Lai, C.M.; Teh, J.; Lin, Y.C.; Liu, Y. Study of a Bidirectional Power Converter Integrated with Battery/Ultracapacitor Dual-Energy Storage. Energies 2020, 13, 1234. [CrossRef]

159. Deng, Y.; Foo, S.Y.; Li, H. Study of Bidirectional DC-DC Converter Interfacing Energy Storage for Vehicle Power Management Using Real Time Digital Simulator (RTDS). J. Power Electron. 2011, 11, 479-489. [CrossRef]

160. Lipu, M.S.H.; Faisal, M.; Ansari, S.; Hannan, M.A.; Karim, T.F.; Ayob, A.; Hussain, A.; Miah, M.S.; Saad, M.H.M. Review of Electric Vehicle Converter Configurations, Control Schemes and Optimizations: Challenges and Suggestions. Electronics 2021, 10, 477. [CrossRef]

161. Eroğlu, F.; Kurtoğlu, M.; Eren, A.; Vural, A.M. Level-Shifted Pulse Width Modulation Based Battery State-of-Charge Balancing Method for Single-Phase Cascaded H-Bridge Multilevel Converters. In Proceedings of the 2021 13th International Conference on Electrical and Electronics Engineering (ELECO), Bursa, Turkey, 25-27 November 2021; pp. 560-564. [CrossRef]

162. Tirpude, S.; Bharadwaj, A.; Dubey, A.; Holmukhe, R.M. Analysis of a Function Fitting Neural Network Based Control Strategy for a Hybrid Energy Storage System Applied to Electric-Vehicles. Int. J. Emerg. Trends Eng. Res. 2020, 8, 2955-2962. [CrossRef]

163. Devi Vidhya, S.; Balaji, M. Failure-Mode Analysis of Modular Multilevel Capacitor-Clamped Converter for Electric Vehicle Application. IET Power Electron. 2019, 12, 3411-3421. [CrossRef]

164. Kolbasov, A.; Kurmaev, R.; Karpukhin, K. Implementation of Dual-Circuit System for Additional Power Supply Based on Photovoltaic Converters for Electric Vehicles. Energies 2019, 12, 4010. [CrossRef]

165. Amjadi, Z.; Williamson, S. Design and Control of a Luo Converter for Variably Loaded Hybrid Electric Vehicle Energy Storage System. Int. J. Power Electron. 2011, 3, 399-416. [CrossRef]

166. Amjadi, Z.; Williamson, S.S. Advanced Digital Control for a Switched Capacitor and Interleaved Switched Capacitor Hybrid Electric Vehicle Energy Management System. Int. J. Electr. Hybrid Veh. 2011, 3, 272-292. [CrossRef]

167. Ebrahimi, J.; Salari, O.; Eren, S.; Hashtrudi-Zaad, K.; Bakhshai, A.; Jain, P. Efficiency Improved Multi-Source Inverter for Hybrid Energy Storage Systems in Electric Vehicle Application. IEEE Trans. Power Electron. 2022, 37, 1982-1997. [CrossRef]

168. Suresh, K.; Sampath, H.; Chellammal, N.; Jondhale, S.R.; Bharatiraja, C. Modular Multi-Input Bidirectional DC to DC Converter for Multi-Source Hybrid Electric Vehicle Applications. J. Appl. Sci. Eng. 2021, 25, 389-399. [CrossRef]

169. De Oliveira-Assis, L.; García-Triviño, P.; Soares-Ramos, E.P.P.; Sarrias-Mena, R.; García-Vázquez, C.A.; Ugalde-Loo, C.E.; Fernández-Ramírez, L.M. Optimal Energy Management System Using Biogeography Based Optimization for Grid-Connected MVDC Microgrid with Photovoltaic, Hydrogen System, Electric Vehicles and Z-Source Converters. Energy Convers. Manag. 2021, 248, 114808. [CrossRef] 
170. Shen, Y.; Li, Y.; Tang, Y.; Sun, J.; Zhao, J.; Yang, X. An Energy Management Strategy Based on Fuzzy Logic for Hybrid Energy Storage System in Electric Vehicles. IEEJ Trans. Electr. Electron. Eng. 2022, 17, 53-60. [CrossRef]

171. Deng, Y.; Foo, S.; Li, H. Real time simulation of power flow control strategies for fuel cell vehicle with energy storage by using real time digital simulator (RTDS). In Proceedings of the 2009 IEEE 6th International Power Electronics and Motion Control Conference, Wuhan, China, 17-20 May 2009; pp. 2323-2327. [CrossRef]

172. Yilmaz, U.; Turksoy, O.; Teke, A. Intelligent Control of High Energy Efficient Two-Stage Battery Charger Topology for Electric Vehicles. Energy 2019, 186, 115825. [CrossRef]

173. Teja, T.S.; Yaseen, M.; Anilkumar, T. Bidirectional DC to DC Converter with ANN Controller for Hybrid Electric Vehicle. Int. J. Innov. Technol. Explor. Eng. 2019, 8, 4446-4453. [CrossRef]

174. Ferreira, A.A.; Pomilio, J.A.; Spiazzi, G.; de Araujo Silva, L. Energy Management Fuzzy Logic Supervisory for Electric Vehicle Power Supplies System. IEEE Trans. Power Electron. 2008, 23, 107-115. [CrossRef]

175. Adam, K.B.; Ashari, M. Design of bidirectional converter using fuzzy logic controller to optimize battery performance in Electric Vehicle. In Proceedings of the International Seminar on Intelligent Technology and Its Applications, Surabaya, Indonesia, 20-21 May 2015; Institute of Electrical and Electronics Engineers Inc.: New York, NY, USA, 2015; pp. 201-205. [CrossRef]

176. Reddy, K.J.; Sudhakar, N. ANFIS-MPPT Control Algorithm for a PEMFC System Used in Electric Vehicle Applications. Int. J. Hydrog. Energy 2019, 44, 15355-15369. [CrossRef]

177. Nguyen, D.D.; Bui, N.T.; Yukita, K. Design and Optimization of Three-Phase Dual-Active-Bridge Converters for Electric Vehicle Charging Stations. Energies 2019, 13, 150. [CrossRef]

178. Prithivi, K.; Sathyapriya, M.; Kumar, A. An Optimized DC-DC Converter for Electric Vehicle Application. Int. J. Mech. Eng. Technol. 2017, 8, 173-182.

179. Surya, S.; Srinivasan, M.K.; Williamson, S. Technological Perspective of Cyber Secure Smart Inverters Used in Power Distribution System: State of the Art Review. Appl. Sci. 2021, 11, 8780. [CrossRef]

180. Xu, J.; Lan, C.; Qiao, Y.; Ma, Y. Prevent Thermal Runaway of Lithium-Ion Batteries with Minichannel Cooling. Appl. Therm. Eng. 2017, 110, 883-890. [CrossRef]

181. Lan, C.; Xu, J.; Qiao, Y.; Ma, Y. Thermal Management for High Power Lithium-Ion Battery by Minichannel Aluminum Tubes. Appl. Therm. Eng. 2016, 101, 284-292. [CrossRef]

182. Xu, J.; Mei, X.; Wang, X.; Fu, Y.; Zhao, Y.; Wang, J. A Relative State of Health Estimation Method Based on Wavelet Analysis for Lithium-Ion Battery Cells. IEEE Trans. Ind. Electron. 2021, 68, 6973-6981. [CrossRef]

183. Xu, J.; Mei, X.; Wang, H.; Wang, J. A Hybrid Self-Heating Method for Batteries Used at Low Temperature. IEEE Trans. Ind. Inform. 2021, 17, 4714-4723. [CrossRef]

184. Mahlia, T.M.I.; Saktisahdan, T.J.; Jannifar, A.; Hasan, M.H.; Matseelar, H.S.C. A Review of Available Methods and Development on Energy Storage; Technology Update. Renew. Sustain. Energy Rev. 2014, 33, 532-545. [CrossRef]

185. Chen, W.; Liang, J.; Yang, Z.; Li, G. A Review of Lithium-Ion Battery for Electric Vehicle Applications and Beyond. Energy Procedia 2019, 158, 4363-4368. [CrossRef]

186. Afif, A.; Rahman, S.M.; Azad, A.T.; Zaini, J.; Islam, M.A.; Azad, A.K. Advanced Materials and Technologies for Hybrid Supercapacitors for Energy Storage-A Review. J. Energy Storage 2019, 25, 100852. [CrossRef]

187. Mekhilef, S.; Saidur, R.; Safari, A. Comparative Study of Different Fuel Cell Technologies. Renew. Sustain. Energy Rev. 2012, 16, 981-989. [CrossRef]

188. Faisal, M.; Hannan, M.A.; Ker, P.J.; Hussain, A.; Mansor, M.B.; Blaabjerg, F. Review of Energy Storage System Technologies in Microgrid Applications: Issues and Challenges. IEEE Access 2018, 6, 35143-35164. [CrossRef]

189. Xavier, L.S.; Amorim, W.C.S.; Cupertino, A.F.; Mendes, V.F.; do Boaventura, W.C.; Pereira, H.A. Power Converters for Battery Energy Storage Systems Connected to Medium Voltage Systems: A Comprehensive Review. BMC Energy 2019, 1, 1-15. [CrossRef] 